ORNL/TM-13500

\title{
Caustic Leaching of Sludges from Selected Hanford Tanks
}

\author{
Barry B. Spencer \\ C. W. Chase \\ B. Z. Egan
}

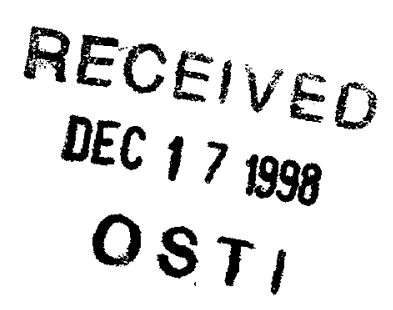

WWMAED ANO OPERATED BY LOCKHEED MARTN EMERGY RESEARCH GORPORATION FOR THE UNTED STATES OEPARIMENT OF ENERGY 
This report has been reproduced from the best available copy.

Reports are available to the public from the following source.

National Technical Information Service

5285 Port Royal Road

Springfield, VA 22161

Telephone 703-605-6000 (1-800-553-6847)

TDD 703-487-4639

Fax 703-605-6900

E-mail orders@ntis.fedworld.gov

Web site hitp://www.ntis.gov/ordering.htm

Reports are available to U.S. Department of Energy (DOE) employees, DOE contractors, Energy Technology Data Exchange (ETDE) representatives, and International Nuclear Information System (INIS) representatives from the following source.

Office of Scientific and Technical Information

P.O. Box 62

Oak Ridge, TN 37831

Telephone 423-576-8401

Fax 423-576-5728

E-mail reports@adonis.osti.gov

Web site http://www.osti.gov/products/sources.html

Reports produced after January 1, 1996, are generally available via the DOE Information Bridge.

Web site http://www.doe.gov/bridge 


\section{DISCLAIMER}

This report was prepared as an account of work sponsored by an agency of the United States Government. Neither the United States Government nor any agency thereof, nor any of their employees, make any warranty, express or implied, or assumes any legal liability or responsibility for the accuracy, completeness, or usefulness of any information, apparatus, product, or process disclosed, or represents that its use would not infringe privately owned rights. Reference herein to any specific commercial product, process, or service by trade name, trademark, manufacturer, or otherwise does not necessarily constitute or imply its endorsement, recommendation, or favoring by the United States Government or any agency thereof. The views and opinions of authors expressed herein do not necessarily state or reflect those of the United States Government or any agency thereof. 


\section{DISCLAIMER}

Portions of this document may be illegible in electronic image products. Images are produced from the best available original document. 
Chemical Technology Division

\title{
CAUSTIC LEACHING OF SLUDGES FROM SELECTED HANFORD TANKS
}

\author{
Barry B. Spencer \\ Robotics and Process Systems Division \\ C. W. Chase \\ Chemical Technology Division
}

\section{B. Z. Egan*}

"Retired from Lockheed Martin Energy Research Corporation; formerly a staff member of the ORNL Chemical Technology Division.

Date Published: August 1998

\author{
Prepared by the \\ OAK RIDGE NATIONAL LABORATORY \\ Oak Ridge, Tennessee 37831-6285 \\ Managed by \\ LOCKHEED MARTIN ENERGY RESEARCH CORP. \\ for the \\ U.S. DEPARTMENT OF ENERGY \\ under contract DE-AC05-960R22464
}





\section{CONTENTS}

Page

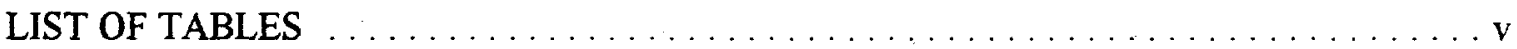

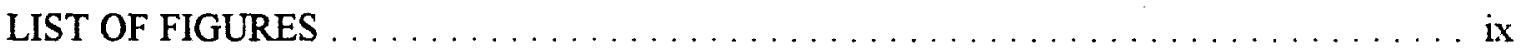

LIST OF ACRONYMS $\ldots \ldots \ldots \ldots \ldots \ldots \ldots \ldots \ldots \ldots \ldots \ldots \ldots \ldots \ldots$

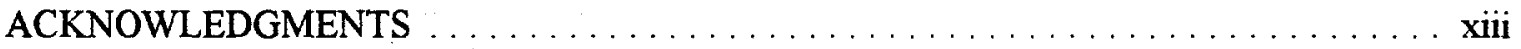

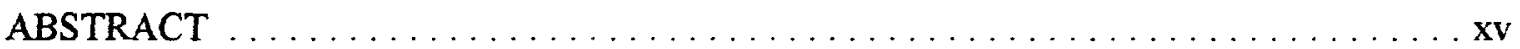

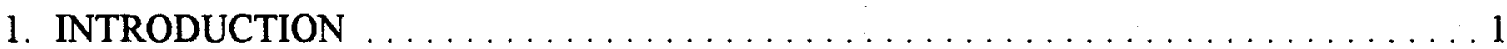

1.1 The Waste Reduction Problem $\ldots \ldots \ldots \ldots \ldots \ldots \ldots \ldots \ldots \ldots$

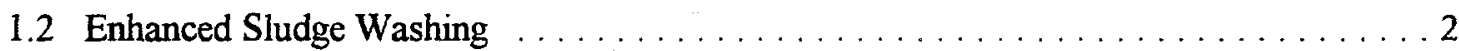

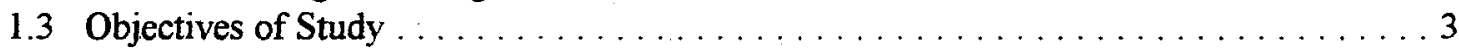

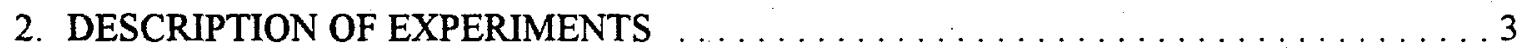

2.1 Parameters of the Enhanced Sludge Washing Process $\ldots \ldots \ldots \ldots \ldots \ldots \ldots$

2.2 Sludge Samples . . . . . . . . . . . . . . . . . . . . . . . . . . 4

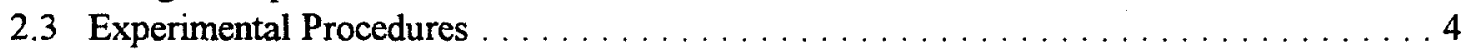

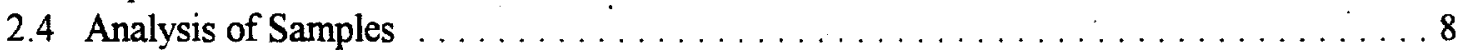

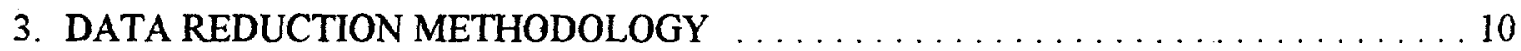

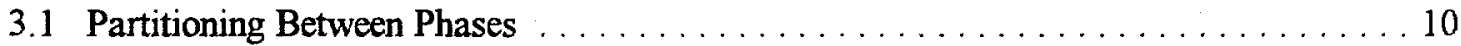

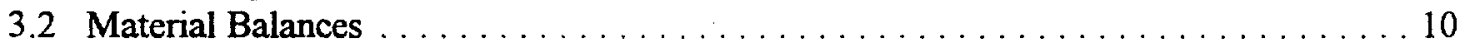

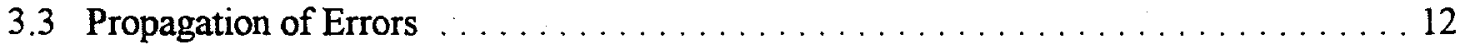

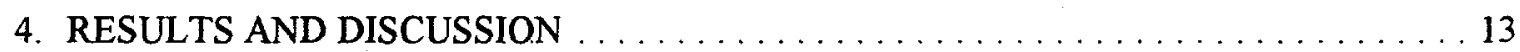

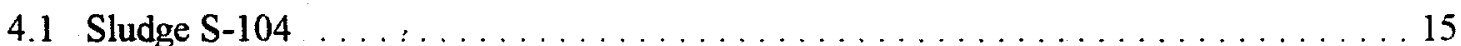

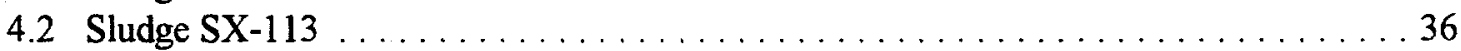

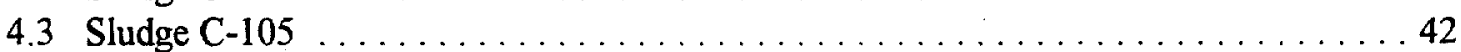

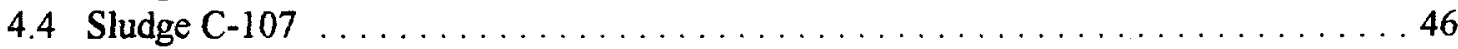

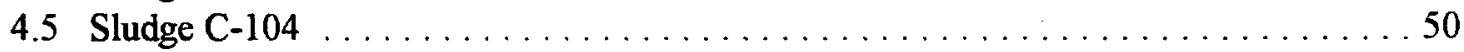

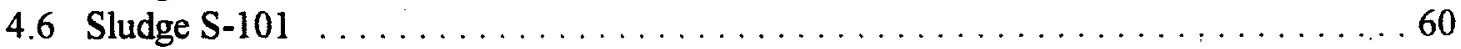

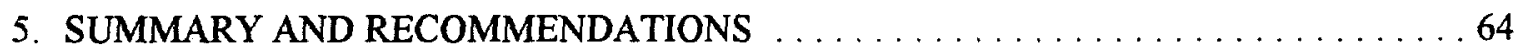

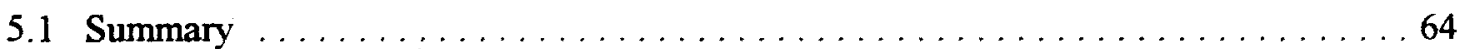

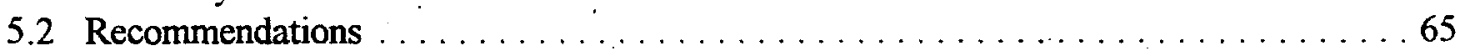

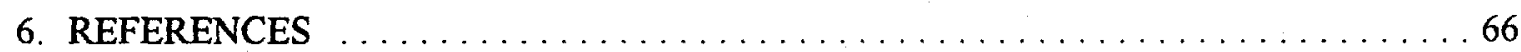





\section{LIST OF TABLES}

Table

Page

2.1 Inventory and some characteristics of sludges that were tested $\ldots \ldots \ldots \ldots \ldots \ldots \ldots$

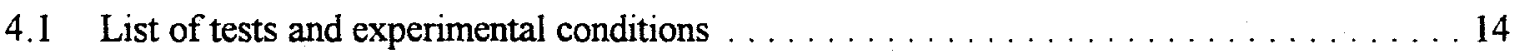

4.2 Amount of sludge used and amount of residue and liquid solutions recovered in each test

4.3 Test \#1 - concentrations of species in enhanced sludge washing process steps for S-104 sludge

4.4 Test \#1 - percentage of each species solubilized and percentage recovery for S-104 sludge

4.5 Test \#2 - concentrations of species in enhanced sludge washing process steps for S-104 sludge

4.6 Test \#2 - percentage of each species solubilized and percentage recovery for S-104 sludge

4.7 Test \#3 - concentrations of species in enhanced sludge washing process steps for S-104 sludge

4.8 Test \#3 - percentage of each species solubilized and percentage recovery for $\mathrm{S}-104$ sludge

4.9 Test \#4 - concentrations of species in enhanced sludge washing process steps for S-104 sludge

4.10 Test \#4 - percentage of each species solubilized and percentage recovery for S-104 sludge

4.11 Test \#5 - concentrations of species in enhanced sludge washing process steps for S-104 sludge

4.12 Test \#5 - percentage of each species solubilized and percentage recovery for S-104 sludge

4.13 Test \#9 - concentrations of species in enhanced sludge washing process steps for S-104 sludge

4.14 Test \#9 - percentage of each species solubilized and percentage recovery for S-104 sludge

4.15 Summary of effects of operating parameters on caustic leaching of S-104 sludge 31 
4.16 Concentration of selected constituents in dry, untreated S-104 sludge determined by different researchers

4.17 Comparison of percentage of selected species removed from S-104 sludge by different researchers

4.18 Test \#6 - concentrations of species in enhanced sludge washing process steps for $\mathrm{SX}-113$ sludge

4.19 Test \#6 - percentage of each species solubilized and percentage recovery for SX-113 sludge

4.20 Test \#10 - concentrations of species in enhanced sludge washing process steps for SX-113 sludge

4.21 Test \#10 - percentage of each species solubilized and percentage recovery for SX-113 sludge

4.22 Effect of operating parameters on caustic leaching behavior of SX-113 sludge

4.23 Concentration of selected constituents in dry, untreated SX-113 sludge determined by different researchers

4.24 Comparison of percentage of selected species removed from SX-113 sludge by different researchers.

4.25 Test \#7 - concentrations of species in enhanced sludge washing process steps for C-105 sludge

4.26 Test \#7 - percentage of each species solubilized and percentage recovery for $\mathrm{C}-105$ sludge

4.27 Concentration of selected constituents in dry, untreated C- 105 sludge determined by different researchers

4.28 Comparison of percentage of selected species removed from C-105 sludge by different researchers.

4.29 Test $\# 8$ - concentrations of species in enhanced sludge washing process steps for $\mathrm{C}-107$ sludge

4.30 Test \#8 - percentage of each species solubilized and percentage recovery for C-107 sludge

4.31 Concentration of selected constituents in dry, untreated C-107 sludge determined by different researchers 
4.32 Comparison of percentage of selected species removed from C-107 sludge by different researchers

4.33 Test \#11 - concentrations of species in enhanced sludge washing process steps for C-104 sludge

4.34 Test \#11 - percentage of each species solubilized and percentage recovery for C-104 sludge

4.35 Test \#12 - concentrations of species in enhanced sludge washing process steps for $\mathrm{C}-104$ sludge

4.36 Test \#12 - percentage of each species solubilized and percentage recovery for C-104 sludge

4.37 Test \#13 - concentrations of species in enhanced sludge washing process steps for $\mathrm{C}$-104 sludge

4.38 Test \#13 - percentage of each species solubilized and percentage recovery

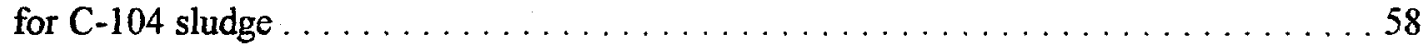

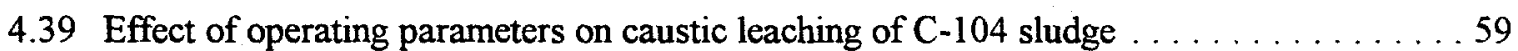

4.40 Concentration of selected constituents in dry, untreated C-104 sludge determined by different researchers

4.41 Comparison of percentage of selected species removed from C-104 sludge by different researchers

4.42 Test \#14 - concentrations of species in enhanced sludge washing process steps for S-101 sludge

4.43 Test \#14 - percentage of each species solubilized and percentage recovery for S-101 sludge

4.44 Concentration of selected constituents in dry, untreated S-101 sludge determined by different researchers

4.45 Comparison of percentage of selected species removed from S-101 sludge by different researchers 



\section{LIST OF FIGURES}

Figure

2.1 Sludge leaching equipment featuring temperature monitor and controller and controlled-temperature mixer

2.2 Up to three samples could be processed simultaneously using this sample tube holder

4.1 The percentage of aluminum removed from S-104 sludge as a function of temperature and caustic concentration

4.2 The percentage of aluminum removed from S-104 sludge as a function of temperature and liquid-to-solids ratio

4.3 Phosphorus follows aluminum removal for S-104 sludge 



\section{LIST OF ACRONYMS}

$\begin{array}{ll}\text { AES } & \text { Atomic Emission Spectroscopy } \\ \text { DOE } & \text { Department of Energy } \\ \text { HLW } & \text { High Level Radioactive Waste } \\ \text { IC } & \text { Ion Chromatography } \\ \text { ICP } & \text { Inductively Coupled Plasma } \\ \text { ICP-AES } & \text { Inductively Coupled Plasma Atomic Emission Spectroscopy } \\ \text { LANL } & \text { Los Alamos National Laboratory } \\ \text { LLW } & \text { Low-Level Radioactive Waste } \\ \text { ORNL } & \text { Oak Ridge National Laboratory } \\ \text { PNNL } & \text { Pacific Northwest National Laboratory } \\ \text { REDOX } & \text { Reduction-Oxidation Process } \\ \text { TRU } & \text { Transuranium }\end{array}$





\section{ACKNOWLEDGMENTS}

This work was sponsored by the U.S. Department of Energy's Office of Science and Technology-Tank Focus Area under U.S. Government contract DE-AC05-960R22464 with Lockheed Martin Energy Research Corp. The work was performed at the Oak Ridge National Laboratory under the auspices of the Chemical Technology Division. The Chemical and Analytical Services Division performed the chemical analyses of samples.

The authors wish to thank their colleagues at Oak Ridge National Laboratory, especially J. M. Giaquinto and J. M. Keller of the Chemistry and Analytical Sciences Division for chemical analyses and E. C. Beahm, J. L. Collins, and T. A. Dillow of the Chemical Technology Division for assistance with the sludge experiments and useful discussions. Technical reviewers were J. L. Collins and T. D. Welch.

Don Temer and his colleagues at Los Alamos National Laboratory and Gregg Lumetta and his colleagues at Pacific Northwest National Laboratory provided valuable information and comments. The authors are also grateful for their assistance in obtaining the Hanford sludge samples. 
( 


\begin{abstract}
Fourteen separate experiments were performed to characterize the caustic leaching behavior of six different Hanford sludges. Six tests were performed with sludge from tank S-104, two tests on SX-113, one test on C-105, one test on C-107, three tests on C-104, and one test on S-101. The test variables were leaching time, leaching temperature, sodium hydroxide concentration in the leach solution, volume of the leaching solution, and mass of the sludge in a batch.

Sludge S-104 is a REDOX waste characterized by average aluminum concentration and high chromium concentration. It was leached with sodium hydroxide solutions ranging in concentration from 3.8 to $6.33 \mathrm{M}$ for durations ranging from 4 to $126 \mathrm{~h}$. More than $95 \%$ of the chromium and cesium was removed under all of the conditions tested. Removal of aluminum improved with increased caustic concentration and increased leaching time, ranging from 20 to $96 \%$ removal. Temperature was varied over a range of 67 to $80^{\circ} \mathrm{C}$, and the percentage of metals removed from the sludge increased with increasing temperature.

Sludge SX-113 is also a REDOX waste characterized as a low aluminum-, low chromiumbearing sludge. Increasing both leaching time and temperature increased the quantities of aluminum, chromium, and cesium removed from the SX-113 sludge with 6.33 $M \mathrm{NaOH}$. Removal of aluminum ranged from 51 to $79 \%$; removal of chromium ranged from 53 to $66 \%$; and removal of cesium ranged from 60 to $86 \%$.

Waste in tank C-105 contains high concentrations of aluminum. Approximately $97 \%$ of the aluminum and $71 \%$ of the chromium were removed from sludge $\mathrm{C}-105$ with $6.33 \mathrm{MNaOH}$ at $70^{\circ} \mathrm{C}$ in $22 \mathrm{~h}$. About $76 \%$ of the cesium was also removed. Similar conditions resulted in the removal of $82 \%$ of the aluminum, $70 \%$ of the chromium, and $73 \%$ of the cesium from sludge C-107, a waste with a high phosphorus concentration.
\end{abstract}


Leaching tests with sludge $\mathrm{C}-104$ were performed at a sodium hydroxide concentration of $4 \mathrm{M}$ and leaching times of $63-65 \mathrm{~h}$. The sludge is high in phosphorus, with moderate concentrations of aluminum and chromium. As operating temperature was increased from 50 to $93^{\circ} \mathrm{C}$, the amount of aluminum removed increased from 29 to $90 \%$ and chromium removal increased from 40 to $74 \%$. Removal of cesium also increased with temperature.

Sludge S-101, a waste with a high chromium concentration, was leached with $4 \mathrm{MNaOH}$ at $93^{\circ} \mathrm{C}$ for $65 \mathrm{~h}$. About $98 \%$ of the aluminum, $86 \%$ of the chromium, and $99 \%$ of the cesium were removed from the sludge.

These results are compared with the results of related tests performed at Los Alamos National Laboratory and Pacific Northwest National Laboratory. In general, any advantages of using higher concentrations of sodium hydroxide can be achieved by increasing the leaching time and temperature, and thereby avoid the disadvantage of handling the additional sodium hydroxide. Differences in results are attributed to differences in leaching procedures, difficulties in chemical analysis, and inhomogeneity of the sludge samples. Additional tests are needed to optimize the caustic leaching parameters.

If it is accepted that these results are applicable to all of the sludge in the six tanks tested (i.e., that these were representative samples), then up to 680 metric tons of aluminum and 20 metric tons of chromium could be removed from the sludge alone by caustic leaching. Significant quantities of other components would also be removed. Therefore, these materials would not have to be included in high-level waste storage. Conditions for optimum removal and separation, especially from transuranium components, still need to be determined. 


\section{INTRODUCTION}

\subsection{THE WASTE REDUCTION PROBLEM}

Production operations at many Department of Energy (DOE) sites throughout the United States have resulted in enormous quantities of radioactive and hazardous wastes which are stored in underground tanks. Most of this waste was produced by the processing of irradiated nuclear fuel to recover uranium and plutonium, and small amounts of waste were produced during research and development activities and during production of isotopes for special (e.g., medical) purposes.

The high-level radioactive waste (HLW) from irradiated fuel processing operations was an acidic aqueous liquid containing the fission products, some of which were highly radioactive, with large amounts of other chemicals added during processing. It also contained small amounts of the actinides, including the transuranium (TRU) elements up through curium. To permit storage of these materials in mild steel tanks, which would otherwise corrode or dissolve in acidic conditions, the waste was neutralized with a base, primarily sodium hydroxide. The addition of sodium hydroxide raised the $\mathrm{pH}$ of the solution (to $\geq 12$ ) and precipitated many of the waste components, creating a sludge. Evaporation of the water to reduce the waste volume resulted in additional precipitation. These processes resulted in wastes comprised of three distinct phases: (1) a high-pH, nitrate-bearing supernatant, (2). a precipitated, actinides-bearing sludge, and (3) an intermediate layer of saltcake. The radioactive components represent only a small fraction of the waste.

If the waste were vitrified without any pretreatment, the resulting glass would be characterized as transuranic HLW and require disposal in a deep geologic repository. The cost of such an option would be prohibitive. Partitioning of the waste into a large low-level radioactive waste (LLW) fraction and a small HLW fraction can greatly reduce disposal costs. The LLW fraction would qualify for near-surface disposal following immobilization (vitrification or grouting), and only the smaller HLW 
fraction would be vitrified and stored in a deep geologic repository. Large savings in the overall disposal costs would be realized.

\subsection{ENHANCED SLUDGE WASHING}

The waste sludges contain high concentrations of nonradioactive materials, such as aluminum, chromium, and phosphates, that can significantly increase the volume of the final $\mathrm{HLW}$ form requiring disposal. There is increasing emphasis on removing these materials using Enhanced Sludge Washing, a process taking advantage of the solubilities of these materials under very caustic conditions to partition the radioactive and nonradioactive components. The behavior of some of the components, such as chromium and phosphate, is also important to vitrification processes. If the nonradioactive components could be preferentially solubilized, then the volume of the remaining radioactive waste to be treated and/or stored would be significantly reduced. The optimum conditions for appropriate partitioning of the sludge components have not been determined.

Aluminum is present in the Hanford tank sludges in large quantities. Caustic leaching of the sludge is expected to solubilize the aluminum by converting it to sodium aluminate (Lumetta et al., 1996); in the case of boehmite,

$$
\mathrm{AlOOH}(s)+\mathrm{NaOH}(a q) \rightarrow \mathrm{NaAlO}_{2}(a q)+\mathrm{H}_{2} \mathrm{O}
$$

and in the case of gibbsite,

$$
\mathrm{Al}(\mathrm{OH})_{3}(s)+\mathrm{NaOH}(a q) \rightarrow \mathrm{NaAlO}_{2}(a q)+2 \mathrm{H}_{2} \mathrm{O}
$$

Metal phosphates in the sludges are expected to react with sodium hydroxide to form soluble sodium orthophosphate (Lumetta et al. 1996 and Lumetta et al. 1997a and 1997b). For example,

$$
\mathrm{FePO}_{4}(s)+3 \mathrm{NaOH}(a q) \rightarrow \mathrm{Fe}(\mathrm{OH})_{3}(s)+\mathrm{Na}_{3} \mathrm{PO}_{4}(a q)
$$


Chromium may be converted to the soluble tetrahydroxochromium(III) anion under conditions of high hydroxide concentration (Lumetta et al., 1996 and Lumetta et al., 1997a and 1997b). That is,

$$
\mathrm{Cr}(\mathrm{OH})_{3}(\mathrm{~s})+\mathrm{NaOH}(a q)-\mathrm{Na}\left[\mathrm{Cr}(\mathrm{OH})_{4}\right](a q)
$$

The relatively poor chromium dissolution of some sludges has led to the study of the alkaline oxidative leaching of sludge to convert the chromium to chromate to improve the dissolution (Rapko et al., 1997).

\subsection{OBJECTIVES OF STUDY}

The objective of this project was to measure the caustic dissolution behavior of sludge components from selected Hanford waste tank sludge samples under different conditions. The dissolution of aluminum, chromium, and other constituents of actual sludge samples in aqueous sodium hydroxide solution was evaluated using various values of temperature, sodium hydroxide concentration, volume of caustic solution per unit mass of sludge (liquid:solids ratio), and leaching time.

\section{DESCRIPTION OF EXPERIMENTS}

\subsection{PARAMETERS OF THE ENHANCED SLUDGE WASHING PROCESS}

The proposed enhanced sludge washing baseline pretreatment would retrieve the sludge by mixing with inhibited water, leach the sludge with caustic, and wash the residue with additional inhibited water to remove all of the leachate solution. The inhibited water contains $0.01 \mathrm{M}$ sodium hydroxide and $0.01 M$ sodium nitrite. In the initial tests conducted by Lumetta et al. (1996), the sludge was leached with $3 \mathrm{M} \mathrm{NaOH}$ for $5 \mathrm{~h}$ at $100^{\circ} \mathrm{C}$. This was expected to remove significant quantities of the aluminum, phosphorus, and chromium. Additional tests by Lumetta et al. (1997b) have been conducted using sequential leach steps and longer leaching times. However, the optimum sodium hydroxide concentration, leach time, leach temperature, and caustic/sludge ratio have not been determined for maximum removal of these components. 


\subsection{SLUDGE SAMPLES}

Samples of sludges from several Hanford tanks were obtained directly from Westinghouse Hanford/Pacific Northwest National Laboratory (PNNL). Other samples were the same samples that were provided to Los Alamos National Laboratory (LANL), and portions were then shipped from LANL to the Oak Ridge National Laboratory (ORNL).

Six sludge samples were selected for these leaching studies. The origin of the samples (specific tank), the type of waste believed to be included in the tank, the total amount of sludge in each tank, and the masses of some of the components of special interest are shown in Table 2.1. Sludges S-101 and S-104 have a high chromium concentration; C-104 and C-107 are high in phosphorus; and $\mathrm{C}-105$ is high in aluminum. Over $8,000,000 \mathrm{~kg}$ of sludge is present in these six tanks, containing over $700,000 \mathrm{~kg}$ of aluminum and $23,000 \mathrm{~kg}$ of chromium.

\subsection{EXPERIMENTAL PROCEDURES}

The leaching experiments were conducted in hot cell A in Building 4501 at ORNL. The cell was cleaned prior to use to minimize any cross contamination. The low contamination level allowed entry into the cell to install and service the equipment. To contain spills, most of the equipment was placed in a large stainless steel tray on a raised platform. Whenever possible, the controls for the equipment were positioned outside the hot cell.

A calibrated Mettler PM4000 top-loading balance with a glass cover was used to weigh the samples. A mixing apparatus was designed and built to leach samples of sludge at temperatures up to $95^{\circ} \mathrm{C}$ (Figs. 2.1 and 2.2). The design allowed high-temperature leaching of up to three sludge samples simultaneously. The centrifuge tubes containing the sludge and leachant were placed in a Teflon holder inside a stainless steel vessel, which was sealed by compressing a Viton gasket between the lip of the vessel and a stainless steel lid. The Teflon holder positioned the centrifuge tubes inside the vessel in a way that prevented direct contact of the tubes with the steel vessel. During a test, about 
Table 2.1. Inventory and some characteristics of sludges that were tested

\begin{tabular}{cccccccc}
\hline Tank & Waste type & $\begin{array}{c}\text { Total mass } \\
(\mathrm{kg})\end{array}$ & $\begin{array}{c}\text { Al mass } \\
(\mathrm{kg})\end{array}$ & $\begin{array}{c}\text { Cr mass } \\
(\mathrm{kg})\end{array}$ & $\begin{array}{c}\text { P mass } \\
(\mathrm{kg})\end{array}$ & $\begin{array}{c}\text { U mass } \\
(\mathrm{kg})\end{array}$ & $\begin{array}{c}\text { Cs-137 } \\
(\mathrm{Ci})\end{array}$ \\
\hline $\mathrm{C}-104^{\mathrm{a}}$ & $\mathrm{PCW}, \mathrm{Zr} \mathrm{CW}$ & $1.34 \times 10^{6}$ & $7.29 \times 10^{4}$ & $1.18 \times 10^{3}$ & $1.76 \times 10^{4}$ & $4.34 \times 10^{4}$ & $8.51 \times 10^{4}$ \\
$\mathrm{C}-105^{\mathrm{a}}$ & $\mathrm{TBP}, \mathrm{Sr}$ & $8.80 \times 10^{5}$ & $1.49 \times 10^{5}$ & $4.10 \times 10^{2}$ & $3.29 \times 10^{3}$ & $1.37 \times 10^{4}$ & $1.61 \times 10^{5}$ \\
${\mathrm{C}-107^{\mathrm{a}}}^{\mathrm{a}}$ & $\mathrm{BiPO}_{4}$ 1C, CW & $1.35 \times 10^{6}$ & $6.39 \times 10^{4}$ & $4.29 \times 10^{2}$ & $6.66 \times 10^{4}$ & $1.43 \times 10^{4}$ & $4.59 \times 10^{4}$ \\
${\mathrm{~S}-101^{\mathrm{a}}}$ & $\mathrm{REDOX}, \mathrm{EB}$ & $2.67 \times 10^{6}$ & $2.37 \times 10^{5}$ & $1.39 \times 10^{4}$ & $1.18 \times 10^{4}$ & $1.38 \times 10^{4}$ & $3.57 \times 10^{5}$ \\
$\mathrm{~S}-104^{\mathrm{b}}$ & REDOX & $1.66 \times 10^{6}$ & $1.96 \times 10^{5}$ & $7.14 \times 10^{3}$ & & $1.10 \times 10^{4}$ & $1.05 \times 10^{5}$ \\
${\mathrm{SX}-113^{\mathrm{a}}}$ & REDOX & $1.38 \times 10^{5}$ & $1.59 \times 10^{3}$ & $8.50 \times 10^{0}$ & & $1.90 \times 10^{1}$ & $3.42 \times 10^{3}$ \\
\hline
\end{tabular}

Key: $\quad$ PCW $=$ PUREX aluminum waste

$\mathrm{Zr} \mathrm{CW}=$ zircaloy cladding waste

$\mathrm{TBP}=$ waste from tributyl phosphate uranium recovery

$\mathrm{Sr}=$ sludge wash waste from strontium extraction process

$\mathrm{BiPO}_{4} 1 \mathrm{C}=$ bismuth phosphate first-cycle decontamination waste

$\mathrm{CW}=$ cladding waste

REDOX $=$ reduction-oxidation process waste

$\mathrm{EB}=$ evaporator bottoms

${ }^{a}$ Source: Colton, N. G., Status Report: Pretreatment Chemistry Evaluation FY 1997 - Wash and

Leach Factors for the Single-shell Tank Waste Inventory, PNNL-1 1646, Battelle, Pacific Northwest

National Laboratory, August 1997.

${ }^{\mathrm{b}}$ Source: Colton, N. G., Status Report: Pretreatment Chemistry Evaluation - Wash and Leach

Factors for the Single-shell Tank Waste Inventory, PNNL-11290, Battelle, Pacific Northwest National

Laboratory, September 1996. 

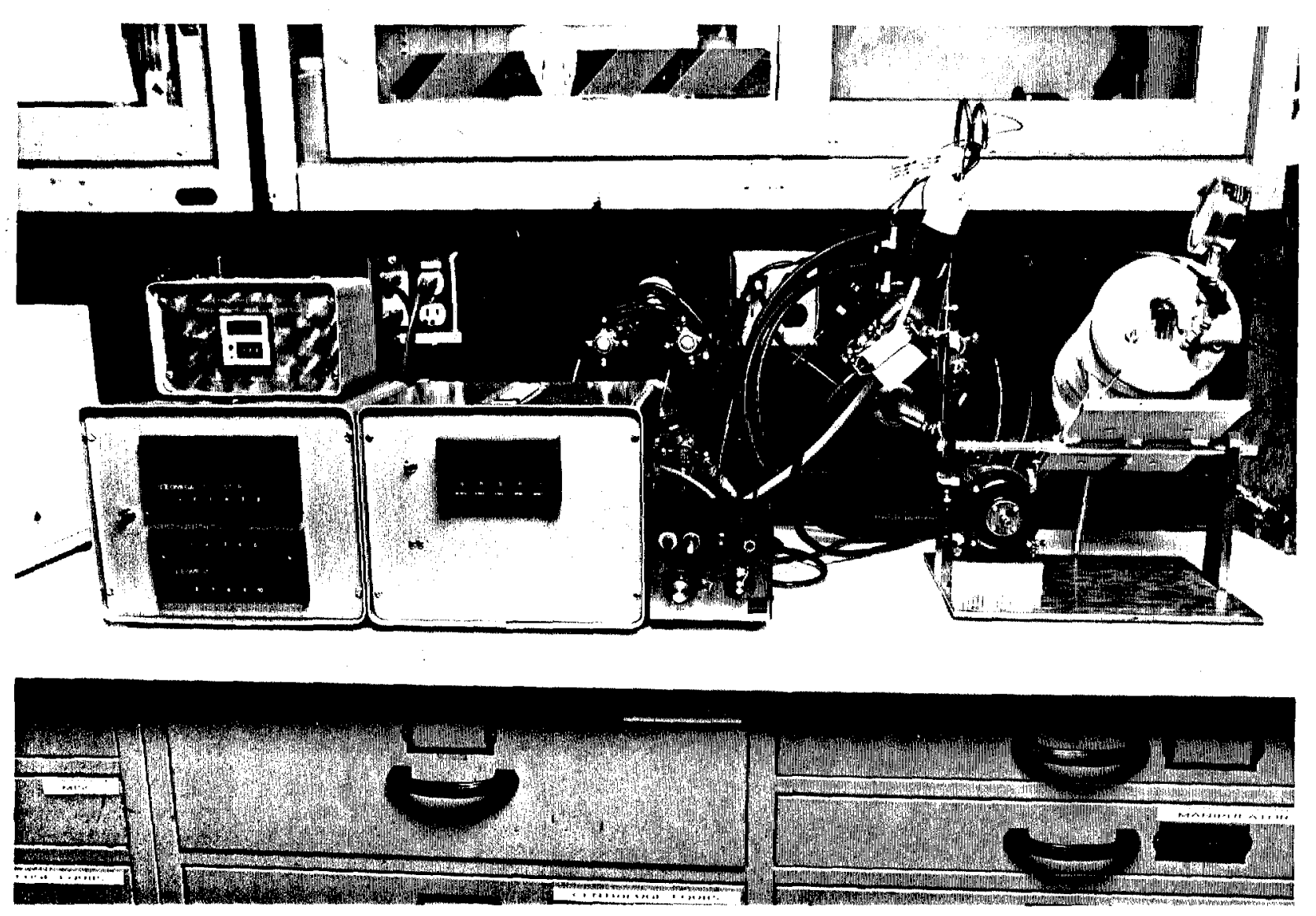

Fig. 2.1. Sludge leaching equipment featuring temperature monitor and controller and controlled-temperature mixer. 


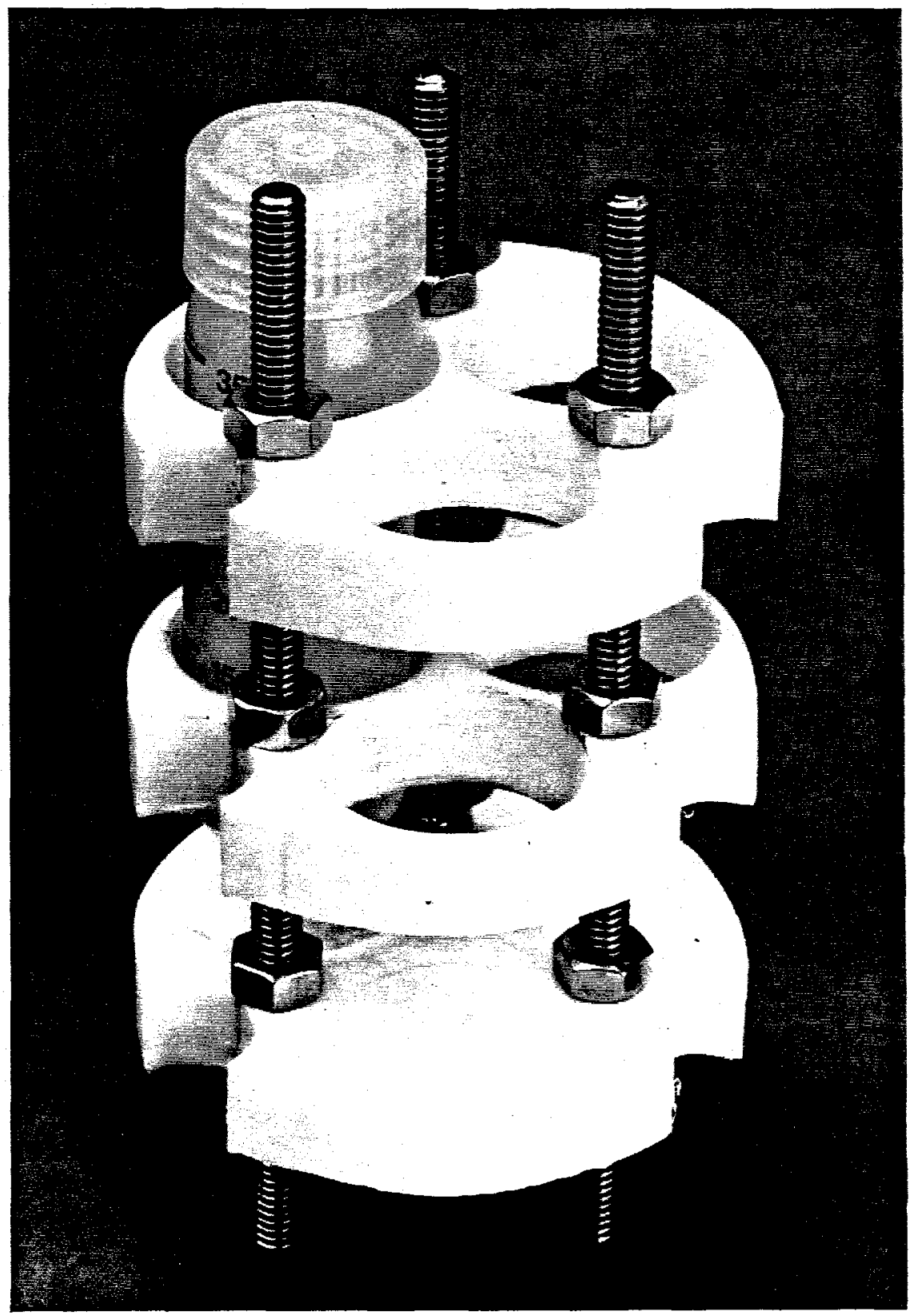

Fig. 2.2. Up to three samples could be processed simultaneously using this sample tube holder. 
half of the volume of the free space in the steel vessel was filled with deionized water to improve heat transfer and temperature control. The temperature inside the vessel was measured with a calibrated J-type thermocouple, which also served as the control measurement. The vessel was placed in a wellinsulated aluminum tube furnace, which was heated with heating tape. Calibrated thermocouples were taped directly to the outside surface of the furnace at three different locations. Two of the thermocouples were monitored with an OMEGA temperature indicator; the other thermocouple was connected to an OMEGA high-temperature controller (Model $\mathrm{CN}-375$ ) and temperature indicator. The furnace assembly was attached to an oscillating mixer that rocked the assembly from $-45^{\circ}$ to $+45^{\circ}$ from the horizontal plane at $\sim 8$ cycles per minute. The centrifuge tubes containing the sludge and leachate were removed from the vessel after the temperature of the heating vessel reached room temperature.

After mixing, the samples were centrifuged for $15-20 \mathrm{~min}$ at $2500 \mathrm{rpm}(\sim 1500 \times$ gravity $)$ using an International Equipment Company Centra-GP8 tabletop centrifuge to separate the sludge solids from wash and leach solutions. The solutions were removed from the centrifuged sludge solids using a specially designed vacuum decantation apparatus. Following the leaching step, the centrifuged, wet sludge residues were rinsed three times with inhibited water, using $\sim 15 \mathrm{~mL}$ of inhibited water for each rinse. A vortex mixer was used to suspend and mix the solids. The residues were again recovered by centrifugation. An ORION Research digital pH meter and an ORION 8103 Ross combination electrode were used for $\mathrm{pH}$ measurements.

\subsection{ANALYSIS OF SAMPLES}

Analytical methods were similar to those described in Appendix B of a report by Sears et al. (1990) and a report by Keller et al. (1996) and are briefly summarized here. Samples of sludge solids were solubilized by microwave-assisted digestion with nitric acid, based on SW-846 Method 3051, Microwave Assisted Acid Digestion of Sediments, Sludges, Soils, and Oils. This method is considered by regulatory agencies to be a total digestion for metals and radionuclides. However, it gave poor 
results for silicon. A simple nitric acid treatment will not dissolve most siliceous materials. Consequently, the residue was assumed to be silicon dioxide, and the silicon was determined gravimetrically.

The leachates and wash solutions were filtered and digested by the SW-846 Method 3015, Microwave Assisted Acid Digestion of Aqueous Samples and Extracts. The leachates and wash solutions were analyzed by the following methods: gross alpha and alpha pulse, gross beta, and gamma spectrometry for radionuclides; inductively coupled plasma atomic emission spectroscopy (ICP-AES) for metals; and ion chromatography for anions. In some samples, bismuth and antimony were determined by Method 7000A, Atomic Absorption Methods. The analytical error for the metal measurements depends upon the analytical method, the concentration level, and the matrix. ICP-AES is a multielement measurement technique designed for the best average performance for all elements and is not optimized for any single component.

The common inorganic anions were measured by ion chromatography (IC) with a Dionex Model $4500 \mathrm{i}$ system. The complex precipitation chemistry of the sludge complicates the measurement of total anions. The primary sludge anion data was based on a water leach, which represented the sum of the anions in the interstitial liquid and the water-soluble anions from the solids. The standard radiochemical methods for radioactive waste characterization are EPA Method 600/900.0, Gross Alpha and Beta Radioactivity in Drinking Water, and EPA Method 600/901.1, Gamma Emitting Radionuclides in Drinking Water. EPA Method 901.1 was used to determine Am-241, Co-60, Cs-134, Cs-137, Eu-152, Eu-154, and Eu-155. Gross beta measurements were made by liquid scintillation counting. In some cases, gross alpha activity was measured and plutonium isotopes were determined by alpha spectrometry after a radiochemical separation.

The moisture content of solids was determined by drying the samples in an oven at $110^{\circ} \mathrm{C}$. Hydroxide concentrations were determined by titration with $0.1 \mathrm{NHCl}$ using phenolphthalein indicator. 


\section{DATA REDUCTION METHODOLOGY}

\subsection{PARTITIONING BETWEEN PHASES}

The caustic leaching studies described in this report are empirical in nature. They were intended to ascertain the extent of the solubility of sludge components under specific conditions. Because the time required to establish an equilibrium was unknown, one could not be sure when the leaching process was complete. As a scoping study, it was sufficient to vary the process conditions (including leaching time) and analyze the products. How each component partitioned between the phases was then quantified.

\subsection{MATERIAL BALANCES}

The data obtained by experiment consisted of the initial mass of the sludge sample, the mass of the residue, the initial and final volumes (or mass and density) of both the leaching and rinsing solutions, and a chemical analysis of the components in each of these four analytes. The percentage of each component removed (e.g., solubilized) from the sludge can be calculated from these data by more than one method. In one method the difference between the quantity of material in the original sludge and that in the residue is the amount removed. The percentage removed is then

$$
w_{i}=\frac{m_{0} C_{0, i}-m_{R} C_{R, i}}{m_{0} C_{0, i}} \times 100,
$$

where

$w_{i}=$ the percentage of species $i$ removed from the sludge by weight;

$m_{0}=$ mass of original sludge sample, $\mathrm{g}$;

$m_{R}=$ mass of sludge residue, $\mathrm{g}$;

$C_{0, i}=$ concentration of component $i$ in original sludge, $\mu \mathrm{g} / \mathrm{g}$;

$C_{R, i}=$ concentration of component $i$ in the sludge residue, $\mu \mathrm{g} / \mathrm{g}$; and 
$i=$ the ith species.

This method is referred to as "based on residual."

Another method supposes that the amount of material removed from the sludge is that which appears in the leachate and rinse solutions. The percentage removed is then

$$
w_{i}^{\prime}=\frac{V_{L} C_{L, i}+V_{W} C_{W, i}}{m_{0} C_{0, i}} \times 100,
$$

where

$$
\begin{aligned}
& w_{i}{ }^{\prime}=\text { the percentage of species } i \text { removed from the sludge by weight (leachate and rinse basis); } \\
& V_{L}=\text { volume (mass) of leachate solution, } \mathrm{mL}(\mathrm{g}) ; \\
& V_{W}=\text { volume (mass) of wash or rinse solution, } \mathrm{mL}(\mathrm{g}) ; \\
& C_{L, i}=\text { concentration of component } i \text { in the leachate, } \mu \mathrm{g} / \mathrm{mL}(\mu \mathrm{g} / \mathrm{g}) ; \text { and } \\
& C_{W, i}=\text { concentration of component } i \text { in the wash solution, } \mu \mathrm{g} / \mathrm{mL}(\mu \mathrm{g} / \mathrm{g}) .
\end{aligned}
$$

Alternate units are given in parenthesis because experimental measurements were made in both units.

An overall material balance was used to evaluate the chemical analyses; that is, the recovery of each species. The percentage recovery is expressed as

$$
f_{i}=\frac{m_{R} C_{R, i}+V_{L} C_{L, i}+V_{W} C_{W, i}}{m_{0} C_{0, i}} \times 100
$$

Values of $f_{i}$ are 100 for a perfect material balance, $\prec 100$ when the amount of $i$ found in the original sludge is greater than the sum of the fractions into which it was partitioned, and $>100$ when the amount of $i$ found in the original sludge is smaller than the sum of the fractions into which it was partitioned. 
It is important to remember that Eqs. (3-1) through (3-3) are valid only if no solutes are introduced by the leaching or rinsing solutions. For example, a strong leaching solution of sodium hydroxide introduces sodium, and the equations must be modified to account for it. The primary interest is in those species, such as aluminum, chromium, phosphate, and a few others, that are not found in the processing solutions. Thus, the equations are adequate for evaluating the data.

\subsection{PROPAGATION OF ERRORS}

Difficulties in measuring the concentration of some species lead to questions regarding how sharply the solubility could be defined. Or, alternatively, how reproducible are the data expected to be?

In the experiments reported in this document, measuring the mass of a sample was easily done to within one part per thousand $(0.1 \%)$. Measurements of sample volume and/or density also approached this accuracy. The largest errors occurred in measuring the concentration of species in the multicomponent mixtures. Errors in measured concentration were often in the $10 \%$ range and were sometimes larger for those species near the lower limit of detection. Therefore, to propagate the errors to the calculated results, it was assumed that the mass (or volume) measurements contained no error and that all of the error originated from the measurement of concentration. Methods to propagate measurement errors to calculated results are given in standard texts (e.g., Holman 1971).

Equation (3-1) was used to calculate the percentage of a species removed from the sludge based on residual analysis. The error in calculating this value was evaluated by

$$
\sigma_{w, i}=\left[\left(\frac{m_{R} C_{R, i}}{m_{0} C_{0, i}^{2}} \sigma_{0, i}\right)^{2}+\left(\frac{m_{R}}{m_{0} C_{0, i}} \sigma_{R, i}\right)^{2}\right]^{1 / 2} \times 100
$$

where

$\sigma_{w, i}=$ error in calculated percentage removed, also expressed in percentage; 


$$
\begin{aligned}
& \sigma_{0, i}=\text { error in measurement of } C_{0, i} \text {, same units as } C_{0, i} ; \text { and } \\
& \sigma_{R, i}=\text { error in measurement of } C_{R, i} \text {, same units as } C_{R, i} \text {. }
\end{aligned}
$$

Similarly, the errors in results calculated by Eq. (3-2) are given by

$$
\sigma_{w^{\prime}, i}=\left[\left(\frac{V_{L}}{m_{0} C_{0, i}} \sigma_{L, i}\right)^{2}+\left(\frac{V_{W}}{m_{0} C_{0, i}} \sigma_{W, i}\right)^{2}+\left(\frac{\left(V_{L} C_{L, i}+V_{W} C_{W, i}\right)}{m_{0} C_{0, i}^{2}} \sigma_{0, i}\right)^{2}\right]^{1 / 2} \times 100
$$

where

$$
\begin{aligned}
& \sigma_{w, i}=\text { error in calculated percentage removed (based on leachate and rinse), percentage: } \\
& \sigma_{L, i}=\text { error in measurement of } C_{\mathrm{L}, i} \text {, same units as } C_{\mathrm{L}, i} ; \text { and } \\
& \sigma_{W, i}=\text { error in measurement of } C_{W, i} \text {, same units as } C_{W, i} .
\end{aligned}
$$

And finally, the errors in results calculated by Eq. (3-3) are given by

$$
\sigma_{f, i}=\left[\left(\frac{m_{R}}{m_{0} C_{0, i}} \sigma_{R, i}\right)^{2}+\left(\frac{V_{L}}{m_{0} C_{0, i}} \sigma_{L, i}\right)^{2}+\left(\frac{V_{W}}{m_{0} C_{0, i}} \sigma_{W, i}\right)^{2}+\left(\frac{\left(m_{R} C_{R, i}+V_{L} C_{L, i}+V_{W} C_{W, i}\right)}{m_{0} C_{0, i}^{2}} \sigma_{0, i}\right)^{2}\right]^{1 / 2} \times 100
$$

where

$\sigma_{f i}=$ error in calculated percentage recovery, percentage.

\section{RESULTS AND DISCUSSION}

Fourteen separate experiments were performed to characterize the leachability of the six different sludges listed in Table 2.1. Six tests were performed with sludge from tank S-104, two tests on SX-113, one test on C-105, one test on C-107, three tests on C-104, and one test on S-101. The leaching time, leaching temperature, sodium hydroxide concentration in the leach solution, volume of leaching solution, and mass of sludge utilized in the tests were varied. Table 4.1 summarizes the conditions at which each test was performed. The weight fraction of the sludge attributable to water is also given in the table. The large variation in water content of the different sludges is evident. The 
Table 4.1. List of tests and experimental conditions

\begin{tabular}{ccccccccc}
\hline $\begin{array}{c}\text { Test } \\
\#\end{array}$ & $\begin{array}{c}\text { Tank } \\
\text { sample }\end{array}$ & $\begin{array}{c}\text { Sludge wt. } \\
(\mathrm{g})\end{array}$ & $\begin{array}{c}\mathrm{NaOH} \text { concn. } \\
(M)\end{array}$ & $\begin{array}{c}\mathrm{NaOH} \text { vol. } \\
(\mathrm{mL})\end{array}$ & $\begin{array}{c}\text { Temp. } \\
\left({ }^{\circ} \mathrm{C}\right)\end{array}$ & $\begin{array}{c}\text { Time } \\
(\mathrm{h})\end{array}$ & $\begin{array}{c}\text { Moisture } \\
(\text { wt } \%)\end{array}$ & $\begin{array}{c}\text { Liquid:solids } \\
(\mathrm{mL} / \mathrm{g})^{\mathrm{a}}\end{array}$ \\
\hline 1 & $\mathrm{~S}-104$ & 3.01 & 3.8 & 23 & 67 & 4 & 15.6 & 9 \\
2 & $\mathrm{~S}-104$ & 3.02 & 3.8 & 23 & 67 & 24 & 15.6 & 9 \\
3 & S-104 & 3.06 & 3.99 & 15 & 70 & 21 & 15.6 & 6 \\
4 & S-104 & 1.49 & 3.99 & 15 & 70 & 21 & 15.6 & 12 \\
5 & S-104 & 1.51 & 6.33 & 15 & 70 & 21 & 15.6 & 12 \\
6 & SX-113 & 1.46 & 6.33 & 15 & 70 & 21 & 47.9 & 20 \\
7 & C-105 & 1.51 & 6.33 & 15 & 70 & 22 & 4.7 & 10 \\
8 & C-107 & 4.37 & 6.33 & 15 & 70 & 22 & 46.3 & 6 \\
9 & S-104 & 1.10 & 6.33 & 30 & 80 & 126 & 15.6 & 32 \\
10 & SX-113 & 1.32 & 6.33 & 30 & 80 & 126 & 47.9 & 44 \\
11 & C-104 & 2.20 & 3.99 & 25 & 80 & 65 & 63.5 & 31 \\
12 & C-104 & 1.44 & 3.99 & 15 & 50 & 63 & 43.7 & 19 \\
13 & C-104 & 2.14 & 3.99 & 21 & 93 & 65 & 63.5 & 27 \\
14 & S-101 & 1.90 & 3.99 & 21 & 93 & 65 & 31.8 & 16 \\
\hline
\end{tabular}

${ }^{\mathrm{a}} \mathrm{Calculated}$ on the basis of the mass of dry sludge: ( $\mathrm{NaOH}$ volume)/[(mass wet sludge)

(1 - \% moisture/100)]. 
volume of solution used to leach the sludge sample and the mass of sludge sample are conveniently combined into the liquid-to-solid ratio, a scaled-parameter that is useful for process design calculations. Because of the variation in the moisture content among the sludges, the liquid:solids ratio was expressed on a dry solids basis, as shown in Table 4.1. Table 4.2 shows the mass of wet residual solids and the mass of the leachate and rinse solutions recovered in each test. The results of the leaching tests are organized by sample origin (i.e., tank name) in the following sections.

\subsection{SLUDGE S-104}

The primary metals found in sludge S-104 are sodium, aluminum, and uranium. Significant concentrations of chromium, iron and phosphorus are also present. (See original sludge analysis in Table 4.3.) Six separate enhanced sludge washing experiments were performed over a range of the operating parameters to ascertain how sludge S-104 would partition among the process streams. Comprehensive listings of the data and calculated results obtained on S-104 sludge are given in Tables 4.3 through 4.14. For a given experiment, the data are provided in one table and the calculated results are provided in the following table. The quantity of each partition or phase has already been given in Table 4.2. For example, the concentration of species measured in the original sludge, residue, leachate solution, and composite rinse solution for test \#1 are given in Table 4.3. The estimated error (standard deviation) in each of these measurements is also listed in the table. Table 4.4 gives the corresponding calculated percentage of each species removed from the sludge and the estimated error in the result. For the purpose of calculation, concentrations below the detectable limit were set to zero.

To illustrate some of the considerations in interpretation of the data and results, discussion of test $\# 1$ is useful. Based on the analysis of the residual solids, $98.0 \% \pm 0.1 \%$ of the chromium is removed from sludge S-104 by leaching with $9 \mathrm{~mL}$ of $3.8 \mathrm{M} \mathrm{NaOH}$ per gram of dry solids at $67^{\circ} \mathrm{C}$ for $4 \mathrm{~h}$. Analysis of the leachate and rinse solutions indicate that $112.8 \% \pm 1.3 \%$ of the chromium is removed. A recovery of $114.8 \% \pm 1.3 \%$ indicates that the chromium material balance is adequate. 
Table 4.2. Amount of sludge used, and amount of residue and liquid solutions recovered in each test

\begin{tabular}{cccccccc}
\hline Test \# & $\begin{array}{c}\text { Tank } \\
\text { sample }\end{array}$ & $\begin{array}{c}\text { Sludge }^{\mathrm{a}} \\
(\mathrm{g})\end{array}$ & $\begin{array}{c}\text { Residue }^{\mathrm{a}} \\
(\mathrm{g})\end{array}$ & $\begin{array}{c}\text { Leachate } \\
(\mathrm{g})\end{array}$ & $\begin{array}{c}\text { Leachate density } \\
(\mathrm{g} / \mathrm{mL})\end{array}$ & $\begin{array}{c}\text { Rinse } \\
(\mathrm{g})\end{array}$ & $\begin{array}{c}\text { Rinse density } \\
(\mathrm{g} / \mathrm{mL})\end{array}$ \\
\hline 1 & S-104 & 3.01 & 3.57 & 27.18 & 1.267 & 44.24 & 1.110 \\
2 & S-104 & 3.02 & 3.29 & 27.10 & 1.273 & 44.17 & 1.109 \\
3 & S-104 & 3.06 & 6.82 & 19.12 & 1.189 & 55.12 & 1.009 \\
4 & S-104 & 1.49 & 4.12 & 18.46 & 1.166 & 57.18 & 1.005 \\
5 & S-104 & 1.51 & 2.33 & 21.96 & 1.230 & 44.10 & 1.0 \\
6 & SX-113 & 1.46 & 1.03 & 20.15 & 1.236 & 45.24 & 1.004 \\
7 & C-105 & 1.51 & 0.53 & 18.79 & 1.238 & 45.99 & 0.999 \\
8 & C-107 & 4.37 & 2.13 & 25.92 & 1.222 & 44.42 & 1.016 \\
9 & S-104 & 1.10 & 0.45 & 36.35 & 1.236 & 42.69 & 1.001 \\
10 & SX-113 & 1.32 & 0.73 & 35.34 & 1.230 & 45.59 & 1.006 \\
11 & C-104 & 2.20 & 2.18 & 28.72 & 1.162 & 48.42 & 1.004 \\
12 & C-104 & 1.44 & 1.57 & 16.50 & 1.154 & 45.54 & 1.008 \\
13 & C-104 & 2.14 & 1.69 & 21.55 & 1.168 & 45.93 & 1.002 \\
14 & S-101 & 1.90 & 0.42 & 21.92 & 1.182 & 47.10 & 0.998 \\
\hline
\end{tabular}

${ }^{\mathrm{a}}$ Mass of centrifuged wet solids following decantation of liquids. 
Table 4.3. Test \#1 - concentrations of species in enhanced sludge washing process steps for S-104 sludge (leach conditions: liquid:solids, $9 \mathrm{~mL} / \mathrm{g}$; $\mathrm{NaOH}, 3.8 \mathrm{M}$; temperature, $67^{\circ} \mathrm{C}$; time, $4 \mathrm{~h}$ )

\begin{tabular}{|c|c|c|c|c|c|c|c|c|}
\hline \multirow[b]{2}{*}{ Species } & \multicolumn{2}{|c|}{$\begin{array}{c}\text { Original sludge } \\
(\mu \mathrm{g} / \mathrm{g})\end{array}$} & \multicolumn{2}{|c|}{$\begin{array}{l}\text { Residual } \\
(\mu \mathrm{g} / \mathrm{g})\end{array}$} & \multicolumn{2}{|c|}{$\begin{array}{l}\text { Leachate } \\
(\mu \mathrm{g} / \mathrm{mL})\end{array}$} & \multicolumn{2}{|c|}{$\begin{array}{c}\text { Rinse } \\
(\mu \mathrm{g} / \mathrm{mL})\end{array}$} \\
\hline & Value & Std deviation & Value & Std deviation & Value & Std deviation & Value. & Std deviation \\
\hline $\mathrm{Ag}$ & $<0.593^{a}$ & $\mathrm{~b}$ & $<1.51$ & $b$ & $<0.774$ & $\mathrm{~b}$ & $<0.0774$ & $\mathrm{~b}$ \\
\hline $\mathrm{Al}$ & 140000 & 1480 & 77300 & 520 & 1200 & 15.8 & 162 & 10.9 \\
\hline $\mathrm{Ba}$ & 29.8 & 0.552 & 18.4 & 0 & $<0.0084$ & $\mathrm{~b}$ & $<0.0084$ & $\mathrm{~b}$ \\
\hline $\mathrm{Be}$ & $<0.0735$ & $\mathrm{~b}$ & $<0.187$ & $b$ & $<0.0096$ & $\mathbf{b}$ & $<0.0096$ & $\mathrm{~b}$ \\
\hline $\mathrm{Bi}$ & 66.8 & 6.89 & 73.3 & 11 & 0.54 & 0.48 & $<0.3$ & $\mathrm{~b}$ \\
\hline $\mathrm{Ca}$ & 256 & 1.47 & 178 & 2.57 & 0.492 & 0.024 & 0.228 & 0.048 \\
\hline $\mathrm{Cd}$ & $<1.01$ & $b$ & $<2.57$ & $\mathrm{~b}$ & $<0.132$ & $b$ & $<0.132$ & $b$ \\
\hline Co & $<0.662$ & $b$ & $<1.69$ & $\mathrm{~b}$ & $<0.0864$ & $b$ & $<0.0864$ & $b$ \\
\hline $\mathrm{Cr}$ & 3100 & 14.2 & 51.5 & 1.4 & 392 & 4.85 & 53.2 & 0.624 \\
\hline $\mathrm{Cu}$ & 47.9 & 0.643 & 13 & 0.468 & 3.82 & 0.132 & 0.036 & 0.012 \\
\hline $\mathrm{Fe}$ & 2480 & 28.3 & 1040 & 6.55 & 0.864 & 0.18 & $<0.0204$ & b \\
\hline $\mathrm{K}$ & 314 & 11.3 & 67.4 & 4.91 & 124 & 44.8 & 42.3 & 0.66 \\
\hline $\mathrm{Mg}$ & 52.6 & 1.75 & 42.9 & 1.64 & $<0.118$ & $b$ & $<0.118$ & $\mathrm{~b}$ \\
\hline $\mathrm{Mn}$ & 1990 & 10.1 & 772 & 5.38 & $<0.0096$ & $b$ & $<0.0096$ & $\mathrm{~b}$ \\
\hline $\mathrm{Ni}$ & 77.3 & 1.29 & 49.6 & 1.64 & $<0.135$ & $\mathrm{~b}$ & $<0.135$ & $\mathrm{~b}$ \\
\hline $\mathrm{P}$ & 2480 & 216 & 720 & 10.1 & $<0.6$ & $b$ & $<0.6$ & $b$ \\
\hline $\mathrm{Sb}$ & $<10.1$ & $b$ & $<25.8$ & $\mathrm{~b}$ & $<1.32$ & $b$ & $<1.32$ & $\mathrm{~b}$ \\
\hline $\mathrm{Si}$ & 134 & 1.1 & 204 & 1.4 & 56 & 1.21 & 7.39 & 0.168 \\
\hline $\mathrm{Sr}$ & 580 & 2.02 & 355 & 0 & 0.102 & 0.012 & $<0.03$ & $\mathrm{~b}$ \\
\hline $\mathrm{Th}$ & 2.67 & 0.827 & $<5.68$ & $\mathrm{~b}$ & 0.372 & 0.312 & $<0.291$ & $\mathrm{~b}$ \\
\hline $\mathrm{Tl}$ & $<8.9$ & $\mathrm{~b}$ & $<22.7$ & $\mathrm{~b}$ & $<1.16$ & $\mathrm{~b}$ & $<1.16$ & b \\
\hline $\mathrm{U}$ & 8400 & 76.8 & 4450 & 17.6 & 1.57 & 0.672 & $<0.547$ & $\mathbf{b}$ \\
\hline $\mathrm{V}$ & $<0.188$ & $\mathrm{~b}$ & $<0.48$ & $\mathrm{~b}$ & $<0.0246$ & b & $<0.0246$ & $b$ \\
\hline $\mathrm{Zn}$ & 11.1 & 1.19 & 8.54 & 2.57 & 1.26 & 0.072 & 0.414 & 0.06 \\
\hline $\mathrm{Br}^{-}$ & $<146$ & $\mathrm{~b}$ & $<502$ & $b$ & $<50$ & $\mathrm{~b}$ & $<5$ & $\mathbf{b}$ \\
\hline $\mathrm{Cl}^{-}$ & 2900 & 30 & $<502$ & $\mathrm{~b}$ & 281 & 2 & 74.9 & 6.8 \\
\hline $\mathrm{F}^{-}$ & $<293$ & $\mathrm{~b}$ & $<502$ & $\mathrm{~b}$ & $<10$ & b & $<10$ & $\mathbf{b}$ \\
\hline $\mathrm{NO}_{3}^{-}$ & 388000 & 9000 & 2030 & 650 & 23400 & 600 & 3600 & 51 \\
\hline $\mathrm{PO}_{4}^{3-}$ & $<585$ & b & $<2008$ & $\mathrm{~b}$ & $<20$ & $b$ & $<20$ & $b$ \\
\hline $\mathrm{SO}_{4}^{2-}$ & 1770 & 220 & $<1004$ & b & 187 & 4 & 30.6 & 4.5 \\
\hline Radiospecies & & $3 \mathrm{~g} / \mathrm{g}$ & & $\mathrm{Bg} / \mathrm{g}$ & & $\mathrm{a} / \mathrm{mL}$ & & $\mathrm{g} / \mathrm{mL}$ \\
\hline Cs- 137 & 2700000 & 100000 & 49000 & 1000 & c & c & c & $\mathrm{c}$ \\
\hline Eu-152 & $<1800$ & $\mathrm{~b}$ & $<1200$ & $b$ & c & c & c & c \\
\hline Eu-154 & 5900 & 1400 & $2900^{\circ}$ & 1500 & c & c & c & c \\
\hline Eu-155 & $<6700$ & $\mathrm{~b}$ & $<3000$ & $\mathrm{~b}$ & c & c & c & c \\
\hline Gross- $\alpha$ & 23000 & 1000 & 16000 & 2000 & $c$ & c & c & c \\
\hline Gross- $\beta$ & 34000000 & 1000000 & 16000000 & 1000000 & $\mathrm{c}$ & $c$ & c & c \\
\hline
\end{tabular}

"The symbol < indicates that the concentration was below the detectable limit given by the following numerical value. 'No standard deviation for values below the detectable limit.

Not measured. 
Table 4.4. Test \#1 - percentage of each species solubilized and percentage recovery for S-104 sludge (leach conditions: liquid:solids, $9 \mathrm{~mL} / \mathrm{g}$; $\mathrm{NaOH}, 3.8 \mathrm{M}$; temperature, $67^{\circ} \mathrm{C}$; time, $4 \mathrm{~h}$ )

\begin{tabular}{|c|c|c|c|c|c|c|}
\hline \multirow[b]{2}{*}{ Species } & \multicolumn{2}{|c|}{$\begin{array}{c}\text { Based on residue } \\
(\%) \\
\end{array}$} & \multicolumn{2}{|c|}{$\begin{array}{c}\text { Based on leachate \& rinse } \\
(\%)\end{array}$} & \multicolumn{2}{|c|}{$\begin{array}{c}\text { Recovery } \\
(\%) \\
\end{array}$} \\
\hline & Value & Std deviation & Value & Std deviation & Value & Std deviation \\
\hline $\mathrm{Ag}$ & a & a & a & a & $\mathrm{a}$ & a \\
\hline $\mathrm{Al}$ & 34.51 & 0.82 & 7.64 & 0.15 & 73.13 & 0.90 \\
\hline $\mathrm{Ba}$ & 26.77 & 1.36 & 0.00 & 0.00 & 73.23 & 1.36 \\
\hline $\mathrm{Be}$ & $\mathbf{a}$ & $\mathrm{a}$ & a & a & $\mathbf{a}$ & $\mathrm{a}$ \\
\hline $\mathrm{Bi}$ & -30.15 & 23.70 & 5.76 & 5.16 & 135.91 & 24.58 \\
\hline $\mathrm{Ca}$ & 17.53 & 1.28 & 2.55 & 0.26 & 85.02 & 1.31 \\
\hline $\mathrm{Cd}$ & $\mathbf{a}$ & a & a & a & a & a \\
\hline $\mathrm{Co}$ & a & a & a & a & a & a \\
\hline $\mathrm{Cr}$ & 98.03 & 0.05 & 112.84 & 1.26 & 114.81 & 1.26 \\
\hline $\mathrm{Cu}$ & 67.81 & 1.24 & 57.83 & 2.14 & 90.02 & 2.60 \\
\hline $\mathrm{Fe}$ & 50.26 & 0.65 & 0.25 & 0.05 & 49.99 & 0.65 \\
\hline $\mathbf{K}$ & 74.54 & 2.07 & 459.81 & 103.05 & 485.27 & 103.22 \\
\hline $\mathrm{Mg}$ & 3.27 & 4.90 & 0.00 & 0.00 & 96.73 & 4.90 \\
\hline $\mathrm{Mn}$ & 53.99 & 0.40 & 0.00 & 0.00 & 46.01 & 0.40 \\
\hline $\mathrm{Ni}$ & 23.90 & 2.82 & 0.00 & 0.00 & 76.10 & 2.82 \\
\hline $\mathrm{P}$ & 65.57 & 3.04 & 0.00 & 0.00 & 34.43 & 3.04 \\
\hline $\mathrm{Sb}$ & $\mathbf{a}$ & a & a & a & $\mathrm{a}$ & a \\
\hline $\mathrm{Si}$ & -80.56 & 1.93 & 370.84 & 7.31 & 551.41 & 8.14 \\
\hline $\mathrm{Sr}$ & 27.41 & 0.25 & 0.13 & 0.01 & 72.72 & 0.25 \\
\hline Th & 100.00 & 0.00 & 99.29 & 88.77 & 99.29 & 88.77 \\
\hline $\mathrm{Tl}$ & $\mathbf{a}$ & $a$ & $a$ & $\mathbf{a}$ & $\mathbf{a}$ & $\mathbf{a}$ \\
\hline $\mathrm{U}$ & 37.17 & 0.63 & 0.13 & 0.06 & 62.97 & 0.63 \\
\hline V & $\mathbf{a}$ & $a$ & a & $\mathrm{a}$ & $\mathbf{a}$ & $\mathbf{a}$ \\
\hline $\mathrm{Zn}$ & 8.75 & 29.15 & 130.28 & 16.36 & 221.53 & 37.29 \\
\hline $\mathrm{Br}^{-}$ & a & a & $\mathrm{a}$ & a & $\mathbf{a}$ & $\mathbf{a}$ \\
\hline $\mathrm{Cl}^{-}$ & 100.00 & 0.00 & 103.25 & 3.32 & 103.25 & 3.32 \\
\hline$F^{-}$ & $\mathbf{a}$ & $\mathbf{a}$ & $\mathbf{a}$ & $\mathbf{a}$ & $\mathbf{a}$ & $\mathbf{a}$ \\
\hline $\mathrm{NO}_{3}^{-}$ & 99.38 & 0.20 & 55.26 & 1.70 & 55.89 & 1.72 \\
\hline $\mathrm{PO}_{4}{ }^{3-}$ & $\mathrm{a}$ & a & $\mathrm{a}$ & $\mathrm{a}$ & $\mathbf{a}$ & $\mathrm{a}$ \\
\hline $\mathrm{SO}_{4}^{2-}$ & 100.00 & 0.00 & 98.18 & 12.76 & 98.18 & 12.76 \\
\hline \multicolumn{7}{|c|}{ Radiospecies } \\
\hline Cs-137 & 97.85 & 0.09 & $b$ & b & 2.15 & 0.09 \\
\hline Eu-152 & $\mathbf{a}$ & a & a . & $\mathbf{a}$ & a & a \\
\hline Eu-154 & 41.70 & 33.18 & $b$ & b & 58.30 & 33.18 \\
\hline Eu-155 & $\mathrm{a}$ & $a$ & a & a & $\mathbf{a}$ & $\mathrm{a}$ \\
\hline Gross- $\alpha$ & 17.49 & 10.92 & b & b & 82.51 & 10.92 \\
\hline Gross- $\beta$ & 44.19 & 3.86 & $b$ & b & 55.81 & 3.86 \\
\hline
\end{tabular}

Insufficient data for calculation; measured concentration in original sludge was below the detectable limit.

Measurement was not made. 
Table 4.5. Test \#2 - concentrations of species in enhanced sludge washing process steps for S-104 sludge (leach conditions: liquid:solids, $9 \mathrm{~mL} / \mathrm{g}$; $\mathrm{NaOH}, 3.8 \mathrm{M}$; temperature, $67^{\circ} \mathrm{C}$; time, $24 \mathrm{~h}$ )

\begin{tabular}{|c|c|c|c|c|c|c|c|c|}
\hline \multirow[b]{2}{*}{ Species } & \multicolumn{2}{|c|}{$\begin{array}{c}\text { Original sludge } \\
(\mu \mathrm{g} / \mathrm{g})\end{array}$} & \multicolumn{2}{|c|}{$\begin{array}{l}\text { Residual } \\
(\mu \mathrm{g} / \mathrm{g})\end{array}$} & \multicolumn{2}{|c|}{$\begin{array}{l}\text { Leachate } \\
(\mu \mathrm{g} / \mathrm{mL})\end{array}$} & \multicolumn{2}{|c|}{$\begin{array}{c}\text { Rinse } \\
(\mu \mathrm{g} / \mathrm{mL})\end{array}$} \\
\hline & Value & Std deviation & Value & Std deviation & Value & Std deviation & Value & Std deviation \\
\hline $\mathrm{Ag}$ & $<0.593^{2}$ & b & $<1.8$ & $\mathrm{~b}$ & $<0.0774$ & $\mathrm{~b}$ & $<0.0774$ & $\mathrm{~b}$ \\
\hline $\mathrm{Al}$ & 140000 & 1480 & 63900 & 731 & 1870 & 12.1 & 292 & 10.9 \\
\hline $\mathrm{Ba}$ & 29.8 & 0.552 & 16 & 0.278 & $<0.0084$ & $\mathrm{~b}$ & $<0.0084$ & $\mathrm{~b}$ \\
\hline $\mathrm{Be}$ & $<0.0735$ & $\mathrm{~b}$ & $<0.223$ & $\mathrm{~b}$ & $<0.0096$ & b & $<0.0096$ & b \\
\hline $\mathrm{Bi}$ & 66.8 & 6.89 & 71.7 & 14.2 & $<0.3$ & $\mathrm{~b}$ & $<0.3$ & $b$ \\
\hline $\mathrm{Ca}$ & 256 & 1.47 & 186 & 1.67 & 0.36 & 0.012 & 0.198 & 0.024 \\
\hline $\mathrm{Cd}$ & $<1.01$ & $\mathrm{~b}$ & $<3.06$ & $b$ & $<0.132$ & $b$ & $<0.132$ & $b$ \\
\hline Co & $<0.662$ & $\mathrm{~b}$ & $<2.01$ & $\mathrm{~b}$ & $<0.0864$ & $b$ & $<0.0864$ & $b$ \\
\hline $\mathrm{Cr}$ & 3100 & 14.2 & 43.2 & 0.557 & 382 & 3.64 & 52.8 & 0.372 \\
\hline $\mathrm{Cu}$ & 47.9 & 0.643 & 8.77 & 0.278 & 3.52 & 0.024 & 0.066 & 0.012 \\
\hline $\mathrm{Fe}$ & 2480 & 28.3 & 940 & 0.835 & 1.21 & 0.084 & $<0.0204$ & b \\
\hline $\mathrm{K}$ & 314 & 11.3 & 19.2 & 6.68 & 116 & 3.64 & 36.9 & 0.468 \\
\hline Mg & 52.6 & 1.75 & 39.1 & 2.23 & $<0.118$ & $b$ & $<0.118$ & $\mathrm{~b}$ \\
\hline $\mathrm{Mn}$ & 1990 & 10.1 & 754 & 0.557 & $<0.0096$ & $\mathrm{~b}$ & $<0.0096$ & $\mathrm{~b}$ \\
\hline $\mathrm{Ni}$ & 77.3 & 1.29 & 46.4 & 1.39 & $<0.135$ & $b$ & $<0.135$ & $b$ \\
\hline $\mathrm{P}$ & 2480 & 216 & 616 & 33.1 & 2.41 & 1.13 & 0.87 & 1.57 \\
\hline $\mathrm{Sb}$ & $<10.1$ & $b$ & $<30.7$ & $\mathrm{~b}$ & $<1.32$ & $\mathbf{b}$ & $<1.32$ & $\mathrm{~b}$ \\
\hline $\mathrm{Si}$ & 134 & 1.1 & 262 & 5.57 & 46.7 & 0.612 & 6.43 & 0.072 \\
\hline Sr & 580 & 2.02 & 271 & 2.23 & 0.072 & 0.012 & $<0.03$ & $\mathrm{~b}$ \\
\hline Th & 2.67 & 0.827 & $<6.75$ & $\mathrm{~b}$ & 0.45 & 0.312 & $<0.291$ & $b$ \\
\hline$\pi 1$ & $<8.9$ & $b$ & $<27$ & $b$ & $<1.16$ & b & $<1.16$ & b \\
\hline$U$ & 8400 & 76.8 & 4160 & 7.52 & 0.894 & 0.348 & $<0.547$ & b \\
\hline $\mathrm{V}$ & $<0.188$ & b & $<0.571$ & $\mathrm{~b}$ & $<0.0246$ & $\mathrm{~b}$ & $<0.0246$ & b \\
\hline $\mathrm{Zn}$ & 11.1 & 1.19 & 8.63 & 2.78 & 1.07 & 0.108 & 0.354 & 0.132 \\
\hline $\mathrm{Br}^{-}$ & $<146$ & $\mathrm{~b}$ & $<300$ & $b$ & $<5$ & $\mathrm{~b}$ & $<5$ & b \\
\hline $\mathrm{Cl}^{-}$ & 2900 & 30 & $<300$ & $\mathrm{~b}$ & 142 & 2 & 74.8 & 4.2 \\
\hline $\mathrm{F}^{-}$ & $<293$ & b & $<300$ & $b$ & $<10$ & $b$ & $<10$ & $\mathbf{b}$ \\
\hline $\mathrm{NO}_{3}^{-}$ & 388000 & 9000 & 1190 & 130 & 5840 & 30 & 4330 & 60 \\
\hline $\mathrm{PO}_{4}{ }^{3-}$ & $<585$ & $\mathrm{~b}$ & $<1200$ & $b$ & $<20$ & $\mathrm{~b}$ & $<20$ & $b$ \\
\hline $\mathrm{SO}_{4}^{2-}$ & 1770 & 220 & $<600$ & $b$ & 82.4 & 15.3 & 35.6 & 2.8 \\
\hline Radiospecies & & $q / g$ & & $B a / g$ & & $\mathrm{~g} / \mathrm{mL}$ & & $\mathrm{g} / \mathrm{ml}$ \\
\hline Cs- 137 & 2700000 & 100000 & 49000 & 2000 & c & $\mathrm{c}$ & c & c \\
\hline Eu-152 & $<1800$ & $\mathrm{~b}$ & $<1400$ & $b$ & c & c & c & c \\
\hline Eu-154 & 5900 & 1400 & 2300 & 800 & c & c & c & c \\
\hline Eu-155 & $<6700$ & $\mathrm{~b}$ & $<3200$ & $b$ & c & c & c & c \\
\hline Gross- $\alpha$ & 23000 & 1000 & 13000 & 2000 & c. & c & c & c \\
\hline Gross $-\beta$ & 34000000 & 1000000 & 17000000 & 1000000 & c & c & c & $c$ \\
\hline
\end{tabular}

"The symbol < indicates that the concentration was below the detectable limit given by the following numerical value. "No standard deviation for values below the detectable limit.

Not measured. 
Table 4.6. Test \#2 - percentage of each species solubilized and percentage recovery for S-104 sludge (leach conditions: liquid:solids, $9 \mathrm{~mL} / \mathrm{g} ; \mathrm{NaOH}, 3.8 \mathrm{M}$; temperature, $67^{\circ} \mathrm{C}$; time, $24 \mathrm{~h}$ )

\begin{tabular}{|c|c|c|c|c|c|c|}
\hline \multirow[b]{2}{*}{ Species } & \multicolumn{2}{|c|}{$\begin{array}{c}\text { Based on residue } \\
(\%) \\
\end{array}$} & \multicolumn{2}{|c|}{$\begin{array}{c}\text { Based on leachate \& rinse } \\
(\%) \\
\end{array}$} & \multicolumn{2}{|c|}{$\begin{array}{c}\text { Recovery } \\
(\%) \\
\end{array}$} \\
\hline & Value & Std deviation & Value & Std deviation & Value & Std deviation \\
\hline $\mathrm{Ag}$ & a & $\mathrm{a}$ & $\mathrm{a}$ & $a$ & $\mathrm{a}$ & $a$ \\
\hline $\mathrm{Al}$ & 50.28 & 0.77 & 12.17 & 0.18 & 61.89 & 0.88 \\
\hline $\mathrm{Ba}$ & 41.51 & 1.49 & 0.00 & 0.00 & 58.49 & 1.49 \\
\hline $\mathrm{Be}$ & $\mathbf{a}$ & a & $\mathbf{a}$ & $\mathrm{a}$ & a & a \\
\hline $\mathrm{Bi}$ & -16.93 & 26.11 & 0.00 & 0.00 & 116.93 & 26.11 \\
\hline $\mathrm{Ca}$ & 20.85 & 0.84 & 2.01 & 0.13 & 81.16 & 0.86 \\
\hline $\mathrm{Cd}$ & $\mathrm{a}$ & a & a & a & a & a \\
\hline Co & $\mathrm{a}$ & $\mathrm{a}$ & $\mathrm{a}$ & $a$ & $\mathbf{a}$ & $\mathbf{a}$ \\
\hline $\mathrm{Cr}$ & 98.48 & 0.02 & 109.33 & 0.98 & 110.85 & 0.98 \\
\hline $\mathrm{Cu}$ & 80.05 & 0.69 & 53.62 & 0.87 & 73.57 & 1.27 \\
\hline $\mathrm{Fe}$ & 58.71 & 0.47 & 0.34 & 0.02 & 41.64 & 0.48 \\
\hline K & 93.34 & 2.33 & 415.42 & 17.15 & 422.08 & 17.51 \\
\hline $\mathrm{Mg}$ & 19.02 & 5.35 & 0.00 & 0.00 & 80.98 & 5.35 \\
\hline $\mathrm{Mn}$ & 58.72 & 0.21 & 0.00 & 0.00 & 41.28 & 0.21 \\
\hline $\mathrm{Ni}$ & 34.61 & 2.24 & 0.00 & 0.00 & 65.39 & 2.24 \\
\hline $\mathrm{P}$ & 72.94 & 2.77 & 1.15 & 0.90 & 28.21 & 2.99 \\
\hline $\mathrm{Sb}$ & $\mathbf{a}$ & $\mathbf{a}$ & $\mathbf{a}$ & a & $\mathrm{a}$ & a \\
\hline $\mathrm{Si}$ & -113.00 & 4.85 & 308.97 & 4.16 & 521.98 & 7.05 \\
\hline Sr & 49.10 & 0.45 & 0.09 & 0.01 & 50.99 & 0.46 \\
\hline Th & 100.00 & 0.00 & 118.81 & 90.22 & 118.81 & 90.22 \\
\hline $\mathrm{Tl}$ & $\mathbf{a}$ & a & a & a & $\mathbf{a}$ & a \\
\hline $\mathrm{U}$ & 46.05 & 0.50 & 0.08 & 0.03 & 54.03 & 0.50 \\
\hline V & $\mathbf{a}$ & $\mathbf{a}$ & a & a & a & a \\
\hline $\mathrm{Zn}$ & 15.30 & 28.76 & 110.02 & 20.79 & 194.72 & 38.38 \\
\hline $\mathrm{Br}^{-}$ & $\mathbf{a}$ & a & a & $a$ & $\mathbf{a}$ & $\mathbf{a}$ \\
\hline $\mathrm{Cl}^{-}$ & 100.00 & 0.00 & 68.54 & 2.09 & 68.54 & 2.09 \\
\hline$F^{-}$ & $\mathrm{a}$ & $\mathrm{a}$ & a & $\mathrm{a}$ & $\mathrm{a}$ & $\mathrm{a}$ \\
\hline $\mathrm{NO}_{3}^{-}$ & 99.67 & 0.04 & 25.33 & 0.62 & 25.66 & 0.63 \\
\hline $\mathrm{PO}_{4}^{3-}$ & a & a & a & a & a & $\mathbf{a}$ \\
\hline $\mathrm{SO}_{4}^{2-}$ & 100.00 & 0.00 & 59.35 & 9.79 & 59.35 & 9.79 \\
\hline \multicolumn{7}{|c|}{ Radiospecies } \\
\hline Cs-137 & 98.02 & 0.11 & $\mathrm{~b}$ & $b$ & 1.98 & 0.11 \\
\hline Eu-152 & $\mathbf{a}$ & $\mathrm{a}$ & $\mathrm{a}$ & $\mathbf{a}$ & $\mathbf{a}$ & $\mathbf{a}$ \\
\hline Eu-154 & 57.53 & 17.88 & b & $b$ & 42.47 & 17.88 \\
\hline Eu-155 & $\mathbf{a}$ & $\mathbf{a}$ & $\mathbf{a}$ & $a$ & $\mathbf{a}$ & $\mathbf{a}$ \\
\hline Gross- $\alpha$ & 38.42 & 9.84 & b & b & 61.58 & 9.84 \\
\hline Gross- $\beta$ & 45.53 & 3.58 & $\mathbf{b}$ & $\mathrm{b}$ & 54.47 & 3.58 \\
\hline
\end{tabular}

'Insufficient data for calculation; measured concentration in original sludge was below the detectable limit.

'Measurement was not made. 
Table 4.7. Test \#3 - concentrations of species in enhanced sludge washing process steps for S-104 sludge (leach conditions: liquid:solids, $6 \mathrm{~mL} / \mathrm{g}$; $\mathrm{NaOH}, 4 \mathrm{M}$; temperature, $70^{\circ} \mathrm{C}$; time, $21 \mathrm{~h}$ )

\begin{tabular}{|c|c|c|c|c|c|c|c|c|}
\hline \multirow[b]{2}{*}{ Species } & \multicolumn{2}{|c|}{$\begin{array}{l}\text { Original sludge } \\
(\mu \mathrm{g} / \mathrm{g})\end{array}$} & \multicolumn{2}{|c|}{$\begin{array}{l}\text { Residual } \\
(\mu \mathrm{g} / \mathrm{g})\end{array}$} & \multicolumn{2}{|c|}{$\begin{array}{l}\text { Leachate } \\
(\mu \mathrm{g} / \mathrm{mL})\end{array}$} & \multicolumn{2}{|c|}{$\begin{array}{c}\text { Rinse } \\
(\mu \mathrm{g} / \mathrm{mL})\end{array}$} \\
\hline & Value & Std deviation & Value & Std deviation & Value & Std deviation & Value & Std deviation \\
\hline $\mathrm{Ag}$ & $<0.593^{\mathrm{a}}$ & b & $<2.89$ & b & $<0.0387$ & $\mathrm{~b}$ & $<0.0387$ & $\mathrm{~b}$ \\
\hline $\mathrm{Al}$ & 140000 & 1480 & 50000 & 404 & 2750 & 3.03 & 68.8 & 7.88 \\
\hline $\mathrm{Ba}$ & 29.8 & 0.552 & 17.7 & 0 & 0.009 & 0 & 0.006 & 0 \\
\hline $\mathrm{Be}$ & $<0.0735$ & $b$ & $<0.358$ & b & $<0.0048$ & $b$ & $<0.0048$ & b \\
\hline $\mathrm{Bi}$ & 66.8 & 6.89 & 88.4 & 14.8 & 0.405 & 0.336 & 0.423 & 0.06 \\
\hline $\mathrm{Ca}$ & 256 & 1.47 & 161 & 1.34 & 0.834 & 0 & $<0.0234$ & $\mathrm{~b}$ \\
\hline $\mathrm{Cd}$ & $<1.01$ & $\mathrm{~b}$ & $<4.92$ & $\mathrm{~b}$ & $<0.066$ & $\mathrm{~b}$ & $<0.066$ & b \\
\hline Co & $<0.662$ & b & $<3.22$ & $\mathrm{~b}$ & $<0.0432$ & $b$ & $<0.0432$ & $b$ \\
\hline $\mathrm{Cr}$ & 3100 & 14.2 & 35.8 & 0.895 & 543 & 3.64 & 13.5 & 0.066 \\
\hline $\mathrm{Cu}$ & 47.9 & 0.643 & 15.7 & 0 & 5.67 & 0.048 & 0.024 & 0.006 \\
\hline $\mathrm{Fe}$ & 2480 & 28.3 & 907 & 4.92 & 1.38 & 0.012 & $<0.0102$ & $\mathrm{~b}$ \\
\hline $\mathrm{K}$ & 314 & 11.3 & $<15.5$ & $\mathrm{~b}$ & 142 & 3.03 & 4.93 & 0.09 \\
\hline $\mathrm{Mg}$ & 52.6 & 1.75 & 32.7 & 1.79 & $<0.0591$ & b & $<0.0591$ & $\mathrm{~b}$ \\
\hline $\mathrm{Mn}$ & 1990 & 10.1 & 762 & 3.58 & $<0.0048$ & $b$ & $<0.0048$ & b \\
\hline $\mathrm{Ni}$ & 77.3 & 1.29 & 41.2 & 1.79 & $<0.0675$ & b & $<0.0675$ & $b$ \\
\hline $\mathrm{P}$ & 2480 & 216 & 620 & 37.6 & 1.12 & 0.426 & 1.59 & 0.42 \\
\hline $\mathrm{Sb}$ & $<10.1$ & $\mathrm{~b}$ & $<49.3$ & $\mathrm{~b}$ & $<0.661$ & $b$ & $<0.661$ & $\mathbf{b}$ \\
\hline $\mathrm{Si}$ & 134 & 1.1 & 545 & 13 & 68.4 & 0.816 & 6.99 & 0.114 \\
\hline $\mathrm{Sr}$ & 580 & 2.02 & 216 & 3.58 & 0.063 & 0 & $<0.015$ & $\mathrm{~b}$ \\
\hline Th & 2.67 & 0.827 & $<10.9$ & $\mathbf{b}$ & 0.6 & 0.06 & $<0.146$ & $b$ \\
\hline $\mathrm{Tl}$ & $<8.9$ & b & $<43.4$ & $\mathbf{b}$ & $<0.581$ & $\mathrm{~b}$ & $<0.581$ & $b$ \\
\hline U & 8400 & 76.8 & 3180 & 41.2 & $<0.273$ & $\mathrm{~b}$ & $<0.273$ & $b$ \\
\hline v & $<0.188$ & $b$ & 1.79 & 0 & $<0.0123$ & $\mathrm{~b}$ & 0.048 & 0.006 \\
\hline $\mathrm{Zn}$ & 11.1 & 1.19 & $<6.6$ & $b$ & 1.08 & 0.084 & $<0.0885$ & $\mathrm{~b}$ \\
\hline $\mathrm{Br}^{-}$ & $<146$ & $\mathrm{~b}$ & $<7.9$ & $b$ & $<50$ & b & $<5$ & $b$ \\
\hline $\mathrm{Cl}^{-}$ & 2900 & 30 & $<9$ & b & 330 & 10 & 17.6 & 5.8 \\
\hline $\mathrm{F}^{-}$ & $<293$ & $\mathrm{~b}$ & $<7.9$ & $\mathrm{~b}$ & $<5$ & $\mathrm{~b}$ & $<5$ & $\mathrm{~b}$ \\
\hline $\mathrm{NO}_{3}^{-}$ & 388000 & 9000 & 433 & 46 & 24700 & 200 & 1010 & 20 \\
\hline $\mathrm{PO}_{4}{ }^{3-}$ & $<585$ & $\mathbf{b}$ & $<15.8$ & $b$ & $<10$ & $\mathbf{b}$ & $<10$ & $\mathrm{~b}$ \\
\hline $\mathrm{SO}_{4}{ }^{2-}$ & 1770 & 220 & 199 & 7 & 332 & 2. & 21.5 & 1.1 \\
\hline
\end{tabular}

\begin{tabular}{|c|c|c|c|c|c|c|c|c|}
\hline \multirow{2}{*}{$\begin{array}{l}\text { Radiospecies } \\
\text { Co-60 }\end{array}$} & \multicolumn{2}{|c|}{$\mathrm{Bg} / \mathrm{g}$} & \multicolumn{2}{|c|}{$\mathrm{Bg} / \mathrm{g}$} & \multicolumn{2}{|c|}{$\mathrm{Bg} / \mathrm{mL}$} & \multicolumn{2}{|c|}{$\mathrm{Bg} / \mathrm{mL}$} \\
\hline & c & c & $<900$ & $\mathrm{~b}$ & $<60$ & $\mathrm{~b}$ & $<100$ & $\mathbf{b}$ \\
\hline Cs-137 & 2700000 & 100000 & 61000 & 3000 & 450000 & 10000 & 12000 & 1000 \\
\hline Eu-152 & $<1800$ & $\mathbf{b}$ & c & c & c & c & c & c \\
\hline Eu-154 & 5900 & 1400 & 3400 & 1100 & c & c & c & c \\
\hline Pu-238 & 380 & c & 184 & c & c & c & c & c \\
\hline $\mathrm{Pu}-239 / 240$ & 19600 & c & 7810 & c & c & c & c & c \\
\hline $\mathrm{Pu}-242$ & 64 & c & 9.6 & $c$ & $\mathrm{c}$ & c & c & c \\
\hline Pu-all & 20000 & 2000 & 8000 & 1300 & $<.2$ & b & 0.28 & 0.64 \\
\hline Gross- $\alpha$ & 23000 & 1000 & 11000 & 3000 & 2.5 & 9 & 5 & 18 \\
\hline Gross- $\beta$ & 34000000 & 1000000 & 12000000 & 1000000 & 520000 & 10000 & 13000 & 1000 \\
\hline
\end{tabular}

${ }^{2}$ The symbol < indicates that the concentration was below the detectable limit given by the following numerical value.

bo standard deviation for values below the detectable limit.

Not measured. 
Table 4.8. Test \#3 - percentage of each species solubilized and percentage recovery for S-104 sludge (leach conditions: liquid:solids, $6 \mathrm{~mL} / \mathrm{g}, \mathrm{NaOH}, 4 \mathrm{M}$; temperature, $70^{\circ} \mathrm{C}$; time, $21 \mathrm{~h}$ )

\begin{tabular}{|c|c|c|c|c|c|c|}
\hline \multirow[b]{2}{*}{ Species } & \multicolumn{2}{|c|}{$\begin{array}{c}\text { Based on residue } \\
(\%) \\
\end{array}$} & \multicolumn{2}{|c|}{$\begin{array}{c}\text { Based on leachate \& rinse } \\
(\%) \\
\end{array}$} & \multicolumn{2}{|c|}{$\begin{array}{l}\text { Recovery } \\
(\%) \\
\end{array}$} \\
\hline & Value & Std deviation & Value & Std deviation & Value & Std deviation \\
\hline $\mathrm{Ag}$ & a & a & a & a & a & a \\
\hline $\mathrm{Al}$ & 20.40 & 1.06 & 11.20 & 0.16 & 90.80 & 1.16 \\
\hline $\mathrm{Ba}$ & -32.38 & 2.45 & 0.52 & 0.01 & 132.90 & 2.46 \\
\hline $\mathrm{Be}$ & a & a & $a$ & $\mathrm{a}$ & a & a \\
\hline $\mathrm{Bi}$ & -194.94 & 58.00 & 14.50 & 3.43 & 309.44 & 58.88 \\
\hline $\mathrm{Ca}$ & -40.17 & 1.42 & 1.71 & 0.01 & 141.88 & 1.42 \\
\hline $\mathrm{Cd}$ & $\mathrm{a}$ & a & $\mathrm{a}$ & a & $\mathrm{a}$ & $\mathrm{a}$ \\
\hline Co & $\mathrm{a}$ & a & a & a & $\mathbf{a}$ & $\mathbf{a}$ \\
\hline $\mathrm{Cr}$ & 97.43 & 0.07 & 99.82 & 0.77 & 102.40 & 0.78 \\
\hline $\mathrm{Cu}$ & 26.95 & 0.98 & 63.10 & 1.02 & 136.15 & 1.92 \\
\hline $\mathrm{Fe}$ & 18.49 & 1.03 & 0.29 & 0.00 & 81.80 & 1.03 \\
\hline $\mathrm{K}$ & 100.00 & 0.00 & 265.68 & 10.83 & 265.68 & 10.83 \\
\hline $\mathrm{Mg}$ & -38.56 & 8.88 & 0.00 & 0.00 & 138.56 & 8.88 \\
\hline $\mathrm{Mn}$ & 14.66 & 0.59 & 0.00 & 0.00 & 85.34 & 0.59 \\
\hline $\mathrm{Ni}$ & -18.79 & 5.53 & 0.00 & 0.00 & 118.79 & 5.53 \\
\hline $\mathrm{P}$ & 44.28 & 5.91 & 1.38 & 0.34 & 57.10 & 6.02 \\
\hline $\mathrm{Sb}$ & $a$ & a & a & $\mathrm{a}$ & $\mathrm{a}$ & a \\
\hline $\mathrm{Si}$ & -806.47 & 22.87 & 361.40 & 4.62 & 1267.87 & 24.26 \\
\hline $\mathrm{Sr}$ & 17.00 & 1.41 & 0.06 & 0.00 & 83.06 & 1.41 \\
\hline Th & 100.00 & 0.00 & 118.09 & 38.44 & 118.09 & 38.44 \\
\hline $\mathrm{Tl}$ & $\mathbf{a}$ & $\mathbf{a}$ & $\mathbf{a}$ & $\mathbf{a}$ & $\mathbf{a}$ & a \\
\hline$U$ & 15.63 & 1.34 & 0.00 & 0.00 & 84.37 & 1.34 \\
\hline V & $\mathrm{a}$ & $\mathbf{a}$ & $\mathrm{a}$ & $\mathrm{a}$ & a & $\mathbf{a}$ \\
\hline $\mathrm{Zn}$ & 100.00 & 0.00 & 51.13 & 6.77 & 51.13 & 6.77 \\
\hline $\mathrm{Br}^{-}$ & a & $\mathbf{a}$ & a & a & a & a \\
\hline $\mathrm{Cl}^{-}$ & 100.00 & 0.00 & 70.64 & 4.07 & 70.64 & 4.07 \\
\hline $\mathrm{F}^{-}$ & $\mathbf{a}$ & $\mathbf{a}$ & $\mathbf{a}$ & a & $\mathbf{a}$ & $\mathbf{a}$ \\
\hline $\mathrm{NO}_{3}^{-}$ & 99.75 & 0.03 & 38.10 & 0.93 & 38.35 & 0.93 \\
\hline $\mathrm{PO}_{4}{ }^{3-}$ & a & $\mathbf{a}$ & a & a & a & $\mathbf{a}$ \\
\hline $\mathrm{SO}_{4}{ }^{2-}$ & 74.94 & 3.24 & 120.26 & 15.00 & 145.32 & 18.13 \\
\hline \multicolumn{7}{|l|}{ Radiospecies } \\
\hline Co-60 & a & $\mathbf{a}$ & a & a & a & $\mathbf{a}$ \\
\hline Cs-137 & 94.96 & 0.31 & 95.52 & 4.09 & 100.55 & 4.26 \\
\hline Eu-152 & $\mathbf{a}$ & a & a & $\mathbf{a}$ & $\mathbf{a}$ & a \\
\hline Eu-154 & -28.44 & 51.53 & $b$ & $b$ & 128.44 & 51.53 \\
\hline Pu-238 & -7.92 & 0.00 & $b$ & b & 107.92 & 0.00 \\
\hline $\mathrm{Pu}-239 / 240$ & 11.19 & 0.00 & b & b & 88.81 & 0.00 \\
\hline $\mathrm{Pu}-242$ & 66.57 & 0.00 & $\mathrm{~b}$ & $\mathrm{~b}$ & 33.43 & 0.00 \\
\hline Pu-all & 10.85 & 17.01 & 0.03 & 0.06 & 89.18 & 17.01 \\
\hline Gross- $\alpha$ & -6.59 & 29.44 & 0.45 & 1.41 & 107.04 & 29.47 \\
\hline Gross- $\boldsymbol{\beta}$ & 21.34 & 6.95 & 8.72 & 0.30 & 87.38 & 7.04 \\
\hline
\end{tabular}


Table 4.9. Test \#4 - concentrations of species in enhanced sludge washing process steps for S-104 sludge (leach conditions: liquid:solids, $12 \mathrm{~mL} / \mathrm{g}$; $\mathrm{NaOH}, 4 \mathrm{M}$; temperature, $70^{\circ} \mathrm{C}$; time, $21 \mathrm{~h}$ )

\begin{tabular}{|c|c|c|c|c|c|c|c|c|}
\hline \multirow[b]{2}{*}{ Species } & \multicolumn{2}{|c|}{$\begin{array}{c}\text { Original sludge } \\
(\mu \mathrm{g} / \mathrm{g})\end{array}$} & \multicolumn{2}{|c|}{$\begin{array}{l}\text { Residual } \\
(\mu g / g)\end{array}$} & \multicolumn{2}{|c|}{$\begin{array}{l}\text { Leachate } \\
(\mu \mathrm{g} / \mathrm{mL})\end{array}$} & \multicolumn{2}{|c|}{$\begin{array}{l}\text { Rinse } \\
(\mu \mathrm{g} / \mathrm{mL})\end{array}$} \\
\hline & Value & Std deviation & Value & Std deviation & Value & td deviation & Value & Std deviation \\
\hline $\mathrm{Ag}$ & $<0.593^{2}$ & $b$ & $<2.88$ & $\mathbf{b}$ & $<0.0387$ & $\mathrm{~b}$ & $<0.0387$ & $b$ \\
\hline $\mathrm{Al}$ & 140000 & 1480 & 40100 & 152 & 1620 & 9.09 & 33.8 & 0.336 \\
\hline $\mathrm{Ba}$ & 29.8 & 0.552 & 15 & 0 & 0.009 & 0 & 0.006 & 0 \\
\hline $\mathrm{Be}$ & $<0.0735$ & b & $<0.358$ & b & $<0.0048$ & $\mathrm{~b}$ & $<0.0048$ & $\mathrm{~b}$ \\
\hline $\mathrm{Bi}$ & 66.8 & 6.89 & 52.1 & 4.47 & 0.534 & 0.192 & 0.666 & 0.288 \\
\hline $\mathrm{Ca}$ & 256 & 1.47 & 118 & 1.34 & 0.903 & 0.018 & 0.027 & 0.006 \\
\hline $\mathrm{Cd}$ & $<1.01$ & $\mathrm{~b}$ & $<4.92$ & $\mathrm{~b}$ & $<0.066$ & $\mathrm{~b}$ & $<0.066$ & b \\
\hline Co & $<0.662$ & $\mathrm{~b}$ & $<3.22$ & $\mathrm{~b}$ & $<0.0432$ & $\mathrm{~b}$ & $<0.0432$ & b \\
\hline $\mathrm{Cr}_{\mathrm{I}}$ & 3100 & 14.2 & 26.8 & 1.34 & 258 & 1.21 & 4.34 & 0.036 \\
\hline $\mathrm{Cu}$ & 47.9 & 0.643 & 13.2 & 0.447 & 2.4 & 0.006 & 0.015 & 0.006 \\
\hline $\mathrm{Fe}$ & 2480 & 28.3 & 783 & 6.26 & 1.36 & 0.012 & $<0.0102$ & b \\
\hline $\mathrm{K}$ & 314 & 11.3 & $<15.5$ & $\mathrm{~b}$ & 109 & 7.27 & 4.27 & 0.264 \\
\hline $\mathrm{Mg}$ & 52.6 & 1.75 & 26.6 & 3.13 & $<0.0591$ & $\mathrm{~b}$ & $<0.0591$ & $\mathrm{~b}$ \\
\hline $\mathrm{Mn}$ & 1990 & 10.1 & 642 & 5.36 & $<0.0048$ & $b$ & $<0.0048$ & $b$ \\
\hline $\mathrm{Ni}$ & 77.3 & 1.29 & 33.5 & 2.24 & $<0.0675$ & b & $<0.0675$ & $\mathrm{~b}$ \\
\hline$P$ & 2480 & 216 & 516 & 15.6 & $<0.3$ & b & 0.903 & 0.666 \\
\hline $\mathrm{Sb}$ & $<10.1$ & b & $<49.2$ & b & $<0.661$ & b & $<0.0661$ & b \\
\hline $\mathrm{Si}$ & 134 & 1.1 & 683 & 8.49 & 59.7 & 0.726 & 6.39 & 0.132 \\
\hline Sr & 580 & 2.02 & 182 & 3.58 & 0.069 & 0 & $<0.015$ & b \\
\hline Th & 2.67 & 0.827 & $<10.8$ & $b$ & 0.405 & 0.108 & $<0.146$ & b \\
\hline $\mathrm{Tl}$ & $<8.9$ & b & $<43.3$ & b & $<0.581$ & $\mathrm{~b}$ & $<0.581$ & b \\
\hline $\mathrm{U}$ & 8400 & 76.8 & 2670 & 20.6 & $<0.273$ & b & $<0.273$ & b \\
\hline v & $<0.188$ & $\mathrm{~b}$ & 1.12 & 1.34 & $<0.0123$ & b & 0.042 & 0 \\
\hline $\mathrm{Zn}$ & 11.1 & 1.19 & $<6.59$ & $\mathbf{b}$ & 0.627 & 0.036 & $<0.0885$ & b \\
\hline $\mathrm{Br}^{-}$ & $<146$ & $\mathrm{~b}$ & $<9.85$ & b & $<50$ & $\mathrm{~b}$ & $<5$ & b \\
\hline $\mathrm{Cl}^{-}$ & 2900 & 30 & $<9.85$ & $\mathrm{~b}$ & 224 & 1 & 7.5 & 0.57 \\
\hline$F^{-}$ & $<293$ & b & $<9.85$ & b & $<5$ & $b$ & $<5$ & $b$ \\
\hline $\mathrm{NO}_{3}^{-}$ & 388000 & 9000 & 230 & 8 & 17500 & 200 & 337 & 34 \\
\hline $\mathrm{PO}_{4}^{3-}$ & $<585$ & $\mathrm{~b}$ & $<19.7$ & $\mathrm{~b}$ & $<10$ & $b$ & $<10$ & $\mathrm{~b}$ \\
\hline $\mathrm{SO}_{4}{ }^{2-}$ & 1770 & 220 & 56.8 & 13.1 & 190 & 15 & 16.4 & 1.3 \\
\hline Radiospecies & & $\mathrm{g} / \mathrm{g}$ & & $B \mathrm{~g} / \mathrm{g}$ & & $\mathrm{mL}$ & & $\mathrm{g} / \mathrm{mL}$ \\
\hline Co-60 & c & c & $<700$ & b & $<40$ & b & $<40$ & b \\
\hline Cs-137 & 2700000 & 100000 & 34000 & 2000 & 230000 & 10000 & 4400 & 300 \\
\hline Eu-154 & 5900 & 1400 & 1600 & 600 & c & c & c & c \\
\hline Pu-238 & 380 & c & 136 & c & c & c & c & c \\
\hline Pu-239/240 & 19600 & c & 6650 & c & c & c & c & c \\
\hline Pu-242 & 64 & c & 13 & c & c & c & c & c \\
\hline Pu-all & 20000 & 2000 & 6800 & 1200 & 0.025 & 0.004 & 0.03 & 0.41 \\
\hline Gross- $\alpha$ & 23000 & 1000 & 9500 & 2700 & $<4$ & $\mathrm{~b}$ & $<$ & b \\
\hline Gross- $\beta$ & 34000000 & 1000000 & 9700000 & 100000 & 260000 & 10000 & 4500 & 100 \\
\hline
\end{tabular}

The symbol < indicates that the concentration was below the detectable limit given by the following numerical value. Wo standard deviation for values below the detectable limit.

Not measured. 
Table 4.10. Test \#4 - percentage of each species solubilized and percentage recovery for S-104 sludge (leach conditions: liquid:solids, $12 \mathrm{~mL} / \mathrm{g}$; $\mathrm{NaOH}, 4 \mathrm{M}$; temperature, $70^{\circ} \mathrm{C}$; time, $21 \mathrm{~h}$ )

\begin{tabular}{|c|c|c|c|c|c|c|}
\hline \multirow[b]{2}{*}{ Species } & \multicolumn{2}{|c|}{$\begin{array}{c}\text { Based on residue } \\
(\%) \\
\end{array}$} & \multicolumn{2}{|c|}{$\begin{array}{c}\text { Based on leachate \& rinse } \\
(\%) \\
\end{array}$} & \multicolumn{2}{|c|}{$\begin{array}{c}\text { Recovery } \\
(\%) \\
\end{array}$} \\
\hline & Value & Std deviation & Value & Std deviation & Value & Std deviation \\
\hline $\mathrm{Ag}$ & $a$ & a & a & a & a & $\mathbf{a}$ \\
\hline $\mathrm{Al}$ & 20.80 & 0.89 & 13.22 & 0.16 & 92.42 & 1.02 \\
\hline $\mathrm{Ba}$ & -39.18 & 2.58 & 1.09 & 0.02 & 140.27 & 2.60 \\
\hline $\mathrm{Be}$ & a & a & $\mathrm{a}$ & $\mathrm{a}$ & $\mathrm{a}$ & a \\
\hline $\mathrm{Bi}$ & -115.66 & 28.93 & 46.55 & 17.41 & 262.21 & 36.80 \\
\hline $\mathrm{Ca}$ & -27.45 & 1.62 & 4.15 & 0.12 & 131.60 & 1.64 \\
\hline $\mathrm{Cd}$ & $\mathbf{a}$ & $\mathrm{a}$ & a & $\mathbf{a}$ & $\mathrm{a}$ & $\mathbf{a}$ \\
\hline Co & $\mathbf{a}$ & $\mathbf{a}$ & a & $\mathbf{a}$ & a & a \\
\hline $\mathrm{Cr}$ & 97.61 & 0.12 & 93.76 & 0.60 & 96.15 & 0.62 \\
\hline $\mathrm{Cu}$ & 23.80 & 2.78 & 54.43 & 0.88 & 130.63 & 3.16 \\
\hline $\mathrm{Fe}$ & 12.70 & 1.22 & 0.58 & 0.01 & 87.88 & 1.22 \\
\hline $\mathrm{K}$ & 100.00 & 0.00 & 420.70 & 29.06 & 420.70 & 29.06 \\
\hline $\mathrm{Mg}$ & -39.83 & 17.10 & 0.00 & 0.00 & 139.83 & 17.10 \\
\hline $\mathrm{Mn}$ & 10.79 & 0.87 & 0.00 & 0.00 & 89.21 & 0.87 \\
\hline $\mathrm{Ni}$ & -19.83 & 8.26 & 0.00 & 0.00 & 119.83 & 8.26 \\
\hline $\mathrm{P}$ & 42.47 & 5.30 & 1.39 & 1.03 & 58.92 & 5.51 \\
\hline $\mathrm{Sb}$ & $\mathbf{a}$ & $\mathbf{a}$ & $a$ & $\mathbf{a}$ & $\mathbf{a}$ & $\mathbf{a}$ \\
\hline $\mathrm{Si}$ & -1309.38 & 20.99 & 655.34 & 8.73 & 2064.72 & 25.33 \\
\hline $\mathrm{Sr}$ & 13.23 & 1.73 & 0.13 & 0.00 & 86.89 & 1.73 \\
\hline Th & 100.00 & 0.00 & 161.15 & 65.87 & 161.15 & 65.87 \\
\hline $\mathrm{TI}$ & $\mathbf{a}$ & a & $\mathrm{a}$ & a & a & $\mathbf{a}$ \\
\hline $\mathrm{U}^{\circ}$ & 12.11 & 1.05 & 0.00 & 0.00 & 87.89 & 1.05 \\
\hline V & $\mathbf{a}$ & $\mathbf{a}$ & $\mathbf{a}$ & $\mathbf{a}$ & $\mathbf{a}$ & $\mathbf{a}$ \\
\hline $\mathrm{Zn}$ & 100.00 & 0.00 & 60.01 & 7.30 & 60.01 & 7.30 \\
\hline $\mathrm{Br}^{-}$ & $\mathbf{a}$ & $a$ & $\mathbf{a}$ & a & $\mathbf{a}$ & $\mathbf{a}$ \\
\hline $\mathrm{Cl}^{-}$ & 100.00 & 0.00 & 91.93 & 1.27 & 91.93 & 1.27 \\
\hline $\mathrm{F}^{-}$ & a & a & $\mathbf{a}$ & a & a & a \\
\hline $\mathrm{NO}_{3}^{-}$ & 99.84 & 0.01 & 51.23 & 1.35 & 51.40 & 1.35 \\
\hline $\mathrm{PO}_{4}^{3-}$ & $\mathrm{a}$ & a & $\mathbf{a}$ & $\mathbf{a}$ & $\mathbf{a}$ & a \\
\hline $\mathrm{SO}_{4}{ }^{2-}$ & 91.13 & 2.32 & 149.41 & 20.83 & 158.28 & 21.91 \\
\hline \multicolumn{7}{|l|}{ Radiospecies } \\
\hline $\mathrm{Co}-60$ & $\mathbf{a}$ & $a$ & $\mathbf{a}$ & $\mathbf{a}$ & $\mathbf{a}$ & $\mathbf{a}$ \\
\hline Cs-137 & 96.52 & 0.24 & 96.72 & 5.34 & 100.20 & 5.43 \\
\hline Eu-154 & 25.01 & 33.28 & b & b & 74.99 & 33.28 \\
\hline $\mathrm{Pu}-238$ & 1.04 & 0.00 & $b$ & $b$ & 98.96 & 0.00 \\
\hline $\mathrm{Pu}-239 / 240$ & 6.18 & 0.00 & $b$ & $\mathrm{~b}$ & 93.82 & 0.00 \\
\hline $\mathrm{Pu}-242$ & 43.83 & 0.00 & b & b & 56.17 & 0.00 \\
\hline Pu-all & 5.99 & 19.07 & 0.01 & 0.08 & 94.02 & 19.07 \\
\hline Gross- $\alpha$ & -14.21 & 32.84 & 0.00 & 0.00 & 114.21 & 32.84 \\
\hline Gross- $\beta$ & 21.11 & 2.46 & 8.63 & 0.40 & 87.52 & 2.72 \\
\hline
\end{tabular}

"Insufficient data for calculation; measured concentration in original sludge was below the detectable limit or was not measured.

Measurement was not made. 
Table 4.11. Test \#5 - concentrations of species in enhanced sludge washing process steps for S-104 sludge (leach conditions: liquid:solids, $12 \mathrm{~mL} / \mathrm{g}$; $\mathrm{NaOH}, 6.33 \mathrm{M}$; temperature, $70^{\circ} \mathrm{C}$; time, $21 \mathrm{~h}$ )

\begin{tabular}{|c|c|c|c|c|c|c|c|c|}
\hline \multirow[b]{2}{*}{ Species } & \multicolumn{2}{|c|}{$\begin{array}{c}\text { Original sludge } \\
(\mu \mathrm{g} / \mathrm{g})\end{array}$} & \multicolumn{2}{|c|}{$\begin{array}{l}\text { Residual } \\
(\mu \mathrm{g} / \mathrm{g})\end{array}$} & \multicolumn{2}{|c|}{$\begin{array}{c}\text { Leachate } \\
(\mu \mathrm{g} / \mathrm{g})\end{array}$} & \multicolumn{2}{|c|}{$\begin{array}{l}\text { Rinse } \\
(\mu \mathrm{g} / \mathrm{g}) \\
\end{array}$} \\
\hline & Value & Std deviation & Value & Std deviation & Value & Std deviation & Value & Std deviation \\
\hline $\mathrm{Ag}$ & $<0.593^{\mathrm{a}}$ & $\mathrm{b}$ & 3.76 & 0.429 & $<0.411$ & b & $<0.507$ & $\mathrm{~b}$ \\
\hline Al & 140000 & 1480 & 66600 & 191 & 1340 & 18.5 & 46.7 & 0.507 \\
\hline $\mathrm{Ba}$ & 29.8 & 0.552 & 28.8 & 0 & $<0.0411$ & $\mathrm{~b}$ & $<0.0507$ & $\mathbf{b}$ \\
\hline $\mathrm{Be}$ & $<0.0735$ & $\mathrm{~b}$ & $<0.107$ & $b$ & $<0.0206$ & $b$ & $<0.0254$ & $\mathrm{~b}$ \\
\hline $\mathrm{Bi}$ & 66.8 & 6.89 & 50.9 & 3.65 & $<8.22$ & $b$ & $<10.1$ & $\mathrm{~b}$ \\
\hline $\mathrm{Ca}$ & 256 & 1.47 & 199 & 0 & 3.25 & 0.123 & 1.14 & 0.101 \\
\hline $\mathrm{Cd}$ & $<1.01$ & $b$ & $<2.15$ & $\mathrm{~b}$ & $<0.411$ & $b$ & $<0.507$ & $b$ \\
\hline Co & $<0.662$ & $\mathbf{b}$ & 2.58 & 3 & $<0.39$ & $b$ & $<0.482$ & $b$ \\
\hline $\mathrm{Cr}$ & 3100 & 14.2 & 58.8 & 0.644 & 170 & 2.14 & 6.24 & 0.0507 \\
\hline $\mathrm{Cu}$ & 47.9 & 0.643 & 20900 & 49.6 & 59.6 & 1.07 & 1.77 & 0.152 \\
\hline $\mathrm{Fe}$ & 2480 & 28.3 & 1590 & 9.44 & 3.97 & 0.123 & 0.761 & 0.0507 \\
\hline $\mathrm{K}$ & 314 & 11.3 & $<10.7$ & $b$ & 59 & 1.44 & 2.66 & 1.37 \\
\hline $\mathrm{Mg}$ & 52.6 & 1.75 & 24.7 & 3 & $<0.514$ & $b$ & $<0.634$ & $b$ \\
\hline $\mathrm{Mn}$ & 1990 & 10.1 & 1280 & 5.58 & 0.0822 & 0 & $<0.0254$ & b \\
\hline $\mathrm{Ni}$ & 77.3 & 1.29 & 71.8 & 1.72 & $<0.411$ & b & $<0.507$ & $b$ \\
\hline $\mathrm{P}$ & 2480 & 216 & 822 & 19.7 & $<2.06$ & b & $<2.54$ & $b$ \\
\hline $\mathrm{Sb}$ & $<10.1$ & $b$ & $<21.5$ & b & $<4.11$ & $b$ & $<5.07$ & $\mathrm{~b}$ \\
\hline $\mathrm{Si}$ & 134 & 1.1 & 6900 & 0 & 61.2 & 1.56 & 36.8 & 0.406 \\
\hline Sr & 580 & 2.02 & 359 & 2.36 & 0.164 & 0 & $<0.127$ & $\mathrm{~b}$ \\
\hline Th & 2.67 & 0.827 & 140 & 4.08 & $<1.03$ & $\mathrm{~b}$ & $<1.27$ & $\mathrm{~b}$ \\
\hline$U$ & 8400 & 76.8 & 5030 & 59 & $<2.06$ & $\mathrm{~b}$ & $<2.54$ & b \\
\hline V & $<0.188$ & $\mathrm{~b}$ & $<1.07$ & $\mathrm{~b}$ & $<0.206$ & $\mathrm{~b}$ & $<0.254$ & $b$ \\
\hline $\mathrm{Zn}$ & 11.1 & 1.19 & 28.9 & 7.94 & 1.01 & 0.288 & $<0.761$ & $\mathrm{~b}$ \\
\hline Radiospecies & \multicolumn{2}{|c|}{$\mathrm{Bg} / \mathrm{g}$} & \multicolumn{2}{|c|}{$\mathrm{Bg} / \mathrm{g}$} & \multicolumn{2}{|c|}{$\mathrm{Bq} / \mathrm{g}$} & \multicolumn{2}{|c|}{$\mathrm{Bq} / \mathrm{g}$} \\
\hline Co-60 & c & c & 190 & 30 & $<6$ & b & $<1$ & $\mathrm{~b}$ \\
\hline Cs-137 & 2700000 & 100000 & 54000 & 1000 & 160000 & 10000 & 5100 & 0 \\
\hline Eu-152 & $<1800$ & $b$ & $<140$ & $\mathrm{~b}$ & $<21$ & $\mathbf{b}$ & $<4$ & b \\
\hline Eu-154 & 5900 & 1400 & 3100 & 200 & $<39$ & $\mathrm{~b}$ & $<3$ & b \\
\hline Eu-155 & $<6700$ & b & $<560$ & $\mathrm{~b}$ & $<110$ & $\mathrm{~b}$ & $<11$ & $\mathrm{~b}$ \\
\hline Pu-238 & 380 & c & 220 & 10 & c & c & $c$ & c \\
\hline $\mathrm{Pu}-239 / 240$ & 19600 & c & 8300 & 300 & c & c & c & c \\
\hline Pu-all & 20000 & 2000 & 8500 & 300 & 2.9 & 2.6 & 0.4 & 1.4 \\
\hline Gross- $\alpha$ & 23000 & 1000 & 17000 & 2000 & 1.4 & 4.7 & 0 & 0.5 \\
\hline Gross- $\beta$ & 34000000 & 1000000 & 19000000 & 1000000 & 200000 & 10000 & 6000 & 100 \\
\hline
\end{tabular}

"The symbol < indicates that the concentration was below the detectable limit given by the following numerical value. 'No standard deviation for values below the detectable limit.

Not measured. 
Table 4.12. Test \#5 - percentage of each species solubilized and percentage recovery for S-104 sludge (leach conditions: liquid:solids, $12 \mathrm{~mL} / \mathrm{g} ; \mathrm{NaOH}, 6.33 \mathrm{M}$; temperature, $70^{\circ} \mathrm{C}$; time, $21 \mathrm{~h}$ )

\begin{tabular}{|c|c|c|c|c|c|c|}
\hline \multirow[b]{2}{*}{ Species } & \multicolumn{2}{|c|}{$\begin{array}{c}\text { Based on residue } \\
(\%) \\
\end{array}$} & \multicolumn{2}{|c|}{$\begin{array}{c}\text { Based on leachate \& rinse } \\
(\%)\end{array}$} & \multicolumn{2}{|c|}{$\begin{array}{c}\text { Recovery } \\
(\%) \\
\end{array}$} \\
\hline & Value & Std deviation & Value & Std deviation & Value & Std deviation \\
\hline $\mathrm{Ag}$ & $\mathbf{a}$ & $\mathrm{a}$ & $\mathbf{a}$ & $\mathbf{a}$ & $\mathbf{a}$ & $\mathbf{a}$ \\
\hline $\mathrm{Al}$ & 26.60 & 0.80 & 14.89 & 0.25 & 88.30 & 0.98 \\
\hline $\mathrm{Ba}$ & -49.13 & 2.76 & 0.00 & 0.00 & 149.13 & 2.76 \\
\hline $\mathrm{Be}$ & $\mathbf{a}$ & $\mathrm{a}$ & a & $\mathrm{a}$ & a & $\mathrm{a}$ \\
\hline $\mathrm{Bi}$ & -17.58 & 14.77 & 0.00 & 0.00 & 117.58 & 14.77 \\
\hline $\mathrm{Ca}$ & -19.95 & 0.69 & 31.47 & 1.36 & 151.42 & 1.60 \\
\hline $\mathrm{Cd}$ & a & a & $\mathbf{a}$ & a & $\mathrm{a}$ & a \\
\hline Co & $\mathbf{a}$ & a & $\mathrm{a}$ & $\mathrm{a}$ & $\mathbf{a}$ & a \\
\hline $\mathrm{Cr}$ & 97.07 & 0.03 & 85.63 & 1.08 & 88.56 & 1.08 \\
\hline $\mathrm{Cu}$ & -67227.07 & 917.80 & 1917.45 & 42.47 & 69244.53 & 943.76 \\
\hline $\mathrm{Fe}$ & 1.07 & 1.27 & 3.22 & 0.10 & 102.15 & 1.31 \\
\hline $\mathrm{K}$ & 100.00 & 0.00 & 298.00 & 17.94 & 298.00 & 17.94 \\
\hline $\mathrm{Mg}$ & 27.54 & 9.12 & 0.00 & 0.00 & 72.46 & 9.12 \\
\hline $\mathrm{Mn}$ & 0.75 & 0.66 & 0.06 & 0.00 & 99.31 & 0.66 \\
\hline $\mathrm{Ni}$ & -43.33 & 4.18 & 0.00 & 0.00 & 143.33 & 4.18 \\
\hline $\mathrm{P}$ & 48.86 & 4.62 & 0.00 & 0.00 & 51.14 & 4.62 \\
\hline $\mathrm{Sb}$ & a & $\mathbf{a}$ & $\mathbf{a}$ & $\mathbf{a}$ & $\mathbf{a}$ & $\mathbf{a}$ \\
\hline $\mathrm{Si}$ & -7845.54 & 65.22 & 1466.26 & 22.58 & 9411.80 & 79.59 \\
\hline $\mathrm{Sr}$ & 4.49 & 0.71 & 0.41 & 0.00 & 95.92 & 0.71 \\
\hline Th & -7990.88 & 2517.12 & 0.00 & 0.00 & 8090.88 & 2517.12 \\
\hline$U$ & 7.60 & 1.37 & 0.00 & 0.00 & 92.40 & 1.37 \\
\hline V & $\mathrm{a}$ & a & a & $\mathrm{a}$ & $\mathrm{a}$ & a \\
\hline $\mathrm{Zn}$ & -301.75 & 118.48 & 132.33 & 40.31 & 534.08 & 129.94 \\
\hline
\end{tabular}

Radiospecies

\begin{tabular}{lccclcc} 
Co-60 & $\mathrm{a}$ & $\mathrm{a}$ & $\mathrm{a}$ & $\mathrm{a}$ & $\mathrm{a}$ & $\mathrm{a}$ \\
Cs-137 & 96.91 & 0.13 & 91.70 & 6.37 & 94.78 & 6.43 \\
Eu-152 & $\mathrm{a}$ & $\mathrm{a}$ & $\mathrm{a}$ & $\mathrm{a}$ & $\mathrm{a}$ & $\mathrm{a}$ \\
Eu-154 & 18.92 & 19.94 & 0.00 & 0.00 & 81.08 & 19.94 \\
Eu-155 & $\mathrm{a}$ & $\mathrm{a}$ & $\mathrm{a}$ & $\mathrm{a}$ & $\mathrm{a}$ & $\mathrm{a}$ \\
Pu-238 & 10.67 & 4.06 & $\mathrm{~b}$ & $\mathrm{~b}$ & 89.33 & 4.06 \\
Pu-239/240 & 34.66 & 2.36 & $\mathrm{~b}$ & $\mathrm{~b}$ & 65.34 & 2.36 \\
Pu-all & 34.42 & 6.95 & 0.27 & 0.28 & 65.85 & 6.99 \\
Gross- $\alpha$ & -14.05 & 14.30 & 0.09 & 0.30 & 114.14 & 14.31 \\
Gross- $\beta$ & 13.77 & 5.20 & 9.07 & 0.50 & 95.30 & 5.35 \\
\hline
\end{tabular}

Insufficient data for calculation; measured concentration in original sludge was below the detectable limit or was not measured.

bMeasurement was not made. 
Table 4.13. Test \#9 - concentrations of species in enhanced sludge washing process steps for S-104 sludge (leach conditions: liquid:solids, $32 \mathrm{~mL} / \mathrm{g} ; \mathrm{NaOH}, 6.33 \mathrm{M}$; temperature, $80^{\circ} \mathrm{C}$; time, $126 \mathrm{~h}$ )

\begin{tabular}{|c|c|c|c|c|c|c|c|c|}
\hline \multirow[b]{2}{*}{ Species } & \multicolumn{2}{|c|}{$\begin{array}{c}\text { Original sludge } \\
(\mu \mathrm{g} / \mathrm{g})\end{array}$} & \multicolumn{2}{|c|}{$\begin{array}{c}\text { Residual } \\
(\kappa \mathrm{g} / \mathrm{g})\end{array}$} & \multicolumn{2}{|c|}{$\begin{array}{c}\text { Leachate } \\
(\mu \mathrm{g} / \mathrm{g}) \\
\end{array}$} & \multicolumn{2}{|c|}{$\begin{array}{r}\text { Rinse } \\
(\mu \mathrm{g} / \mathrm{g}) \\
\end{array}$} \\
\hline & Value & Std deviation & Value & Std deviation & Value & Std deviation & Value & Std deviation \\
\hline $\mathrm{Ag}$ & $<0.593^{\mathrm{a}}$ & $b$ & $<2.20$ & b & $<0.0804$ & b & $<0.103$ & b \\
\hline $\mathrm{Al}$ & 140000 & 1480 & 12600 & 125 & 3830 & 25.2 & 45.5 & 4.55 \\
\hline $\mathrm{Ba}$ & 29.8 & 0.552 & 99 & 0.44 & $<0.00804$ & $\mathbf{b}$ & $<0.0103$ & b \\
\hline $\mathrm{Be}$ & $<0.0735$ & $b$ & $<0.11$ & $b$ & $<0.00402$ & b & $<0.00513$ & $b$ \\
\hline $\mathrm{Bi}$ & 66.8 & 6.89 & 2.18 & 0.198 & $<0.0603$ & $b$ & $<0.0257$ & $b$ \\
\hline $\mathrm{Ca}$ & 256 & 1.47 & 599 & 1.32 & $<0.0322$ & b & 0.395 & 0.0395 \\
\hline $\mathrm{Cd}$ & $<1.01$ & b & $<2.20$ & b & $<0.0804$ & $\mathbf{b}$ & $<0.103$ & b \\
\hline Co & $<0.662$ & $\mathbf{b}$ & 4.95 & 0.88 & $<0.0764$ & $\mathbf{b}$ & $<0.0975$ & $\mathbf{b}$ \\
\hline $\mathrm{Cr}$ & 3100 & 14.2 & 68 & 0.44 & 84.4 & 2.44 & 1.03 & 0.103 \\
\hline $\mathrm{Cu}$ & 47.9 & 0.643 & 27.4 & 0.88 & $<0.0161$ & $\mathrm{~b}$ & 0.133 & 0.0133 \\
\hline $\mathrm{Fe}$ & 2480 & 28.3 & 3040 & 9.24 & 23.4 & 0.346 & 0.149 & 0.0149 \\
\hline K & 314 & 11.3 & $<11.0$ & $\mathbf{b}$ & 182 & 7.24 & 0.903 & 0.215 \\
\hline $\mathrm{Mg}$ & 52.6 & 1.75 & 191 & 5.28 & $<0.100$ & b & $<0.128$ & $b$ \\
\hline $\mathrm{Mn}$ & 1990 & 10.1 & 4460 & 13.9 & 0.0723 & 0 & $<0.00513$ & $b$ \\
\hline $\mathrm{Ni}$ & 77.3 & 1.29 & 268 & 13.9 & $<0.0804$ & $b$ & $<0.103$ & $\mathrm{~b}$ \\
\hline$P$ & 2480 & 216 & 224 & 64.9 & 0.655 & 1.3 & $<0.513$ & $\mathrm{~b}$ \\
\hline $\mathrm{Sb}$ & $<10.1$ & $\mathbf{b}$ & $<22.0$ & $b$ & $<0.804$ & $\mathrm{~b}$ & $<0.0257$ & $b$ \\
\hline $\mathrm{Si}$ & 134 & 1.1 & 4900 & 0 & c & $\mathrm{c}$ & c & $\mathrm{c}$ \\
\hline $\mathrm{Sr}$ & 580 & 2.02 & 1160 & 13.9 & 1.58 & 0.0402 & $<0.0257$ & $b$ \\
\hline Th & 2.67 & 0.827 & $<231$ & $b$ & 0.354 & 0.498 & $<0.257$ & $b$ \\
\hline $\mathrm{U}$ & 8400 & 76.8 & 19900 & 240 & 0.599 & 0.402 & $<0.513$ & $b$ \\
\hline $\mathrm{V}$ & $<0.188$ & b & $<1.10$ & b & 0.113 & 0.0161 & $<0.0513$ & $b$ \\
\hline $\mathrm{Zn}$ & 11.1 & 1.19 & 15.1 & 2.42 & $<0.121$ & $\mathbf{b}$ & $<0.154$ & b \\
\hline Radiospecies & \multicolumn{2}{|c|}{$\mathrm{Bq} / \mathrm{g}$} & \multicolumn{2}{|c|}{$\mathrm{Bg} / \mathrm{g}$} & \multicolumn{2}{|c|}{$\mathrm{Bg} / \mathrm{g}$} & \multicolumn{2}{|c|}{$\mathrm{Bg} / \mathrm{g}$} \\
\hline Co-60 & c & c & c & c & 6.28 & 0 & 15.1 & 0 \\
\hline Cs-137 & 2700000 & 100000 & 150000 & 10000 & 86000 & 1000 & 980 & 80 \\
\hline Eu-152 & $<1800$ & b & c & c & $<170$ & $\mathbf{b}$ & $<31$ & $b$ \\
\hline Eu-154 & 5900 & 1400 & c & c & $<120$ & $\mathbf{b}$ & $<67$ & $\mathrm{~b}$ \\
\hline Eu-155 & $<6700$ & $\mathrm{~b}$ & c & c & $<410$ & $\mathbf{b}$ & $<49$ & $\mathbf{b}$ \\
\hline Pu-238 & 380 & c & 1200 & 100 & c & $\mathbf{c}$ & $\mathbf{c}$ & c \\
\hline $\mathrm{Pu}-239 / 240$ & 19600 & c & 43000 & 1000 & c & c & c & c \\
\hline Gross- $\alpha$ & 23000 & 1000 & 57000 & 3000 & $<1.60$ & $b$ & & \\
\hline Gross- $\boldsymbol{\beta}$ & 34000000 & 1000000 & 64000000 & 1000000 & 180000 & 10000 & 1200 & 100 \\
\hline
\end{tabular}

The symbol < indicates that the concentration was below the detectable limit given by the following numerical value. Wo standard deviation for values below the detectable limit.

Not measured. 
Table 4.14. Test \#9 - percentage of each species solubilized and percentage recovery for S-104 sludge (leach conditions: liquid:solids, $32 \mathrm{~mL} / \mathrm{g} ; \mathrm{NaOH}, 6.33 \mathrm{M}$; temperature, $80^{\circ} \mathrm{C}$; time, $126 \mathrm{~h}$ )

\begin{tabular}{|c|c|c|c|c|c|c|}
\hline \multirow[b]{2}{*}{ Species } & \multicolumn{2}{|c|}{$\begin{array}{c}\text { Based on residue } \\
(\%) \\
\end{array}$} & \multicolumn{2}{|c|}{$\begin{array}{c}\text { Based on leachate \& rinse } \\
(\%)\end{array}$} & \multicolumn{2}{|c|}{$\begin{array}{c}\text { Recovery } \\
(\%) \\
\end{array}$} \\
\hline & Value & Std deviation & Value & Std deviation & Value & Std deviation \\
\hline $\mathrm{Ag}$ & $\mathbf{a}$ & $\mathbf{a}$ & $\mathbf{a}$ & $\mathbf{a}$ & $\mathbf{a}$ & $\mathrm{a}$ \\
\hline $\mathrm{Al}$ & 96.32 & 0.05 & 91.66 & 1.14 & 95.35 & 1.18 \\
\hline $\mathrm{Ba}$ & -35.91 & 2.59 & 0.00 & 0.00 & 135.91 & 2.59 \\
\hline $\mathrm{Be}$ & a & a & a & a & a & a \\
\hline $\mathrm{Bi}$ & 98.66 & 0.18 & 0.00 & 0.00 & 1.34 & 0.18 \\
\hline $\mathrm{Ca}$ & 4.28 & 0.59 & 5.99 & 0.60 & 101.71 & 0.86 \\
\hline $\mathrm{Cd}$ & a & $\mathbf{a}$ & a & a & $\mathrm{a}$ & a \\
\hline $\mathrm{Co}$ & $\mathrm{a}$ & $\mathbf{a}$ & $\mathbf{a}$ & a & a & $\mathbf{a}$ \\
\hline $\mathrm{Cr}$ & 99.10 & 0.01 & 91.26 & 2.64 & 92.16 & 2.64 \\
\hline $\mathrm{Cu}$ & 76.60 & 0.81 & 10.78 & 1.09 & 34.18 & 1.39 \\
\hline $\mathrm{Fe}$ & 49.85 & 0.59 & 31.41 & 0.58 & 81.56 & 1.05 \\
\hline $\mathrm{K}$ & 100.00 & 0.00 & 1926.53 & 103.05 & 1926.53 & 103.05 \\
\hline $\mathrm{Mg}$ & -48.55 & 6.43 & 0.00 & 0.00 & 148.55 & 6.43 \\
\hline Mn & 8.31 & 0.55 & 0.12 & 0.00 & 91.81 & 0.55 \\
\hline $\mathrm{Ni}$ & -41.83 & 7.73 & 0.00 & 0.00 & 141.83 & 7.73 \\
\hline $\mathrm{P}$ & 96.30 & 1.12 & 0.87 & 1.73 & 4.57 & 2.07 \\
\hline $\mathrm{Sb}$ & a & $\mathrm{a}$ & a & a & $\mathbf{a}$ & $\mathbf{a}$ \\
\hline $\mathrm{Si}$ & -1395.93 & 12.28 & 0.00 & 0.00 & 1495.93 & 12.28 \\
\hline $\mathrm{Sr}$ & 18.18 & 1.02 & 9.00 & 0.23 & 90.82 & 1.06 \\
\hline Th & 100.00 & 0.00 & 438.13 & 631.12 & 438.13 & 631.12 \\
\hline $\mathrm{U}$ & 3.08 & 1.47 & 0.24 & 0.16 & 97.15 & 1.48 \\
\hline V & a & $\mathbf{a}$ & a & $\mathbf{a}$ & $\mathbf{a}$ & $\mathrm{a}$ \\
\hline $\mathrm{Zn}$ & 44.35 & 10.73 & 0.00 & 0.00 & 55.65 & 10.73 \\
\hline \multicolumn{7}{|l|}{ Radiospecies } \\
\hline $\mathrm{Co}-60$ & a & a & a & a & $\mathbf{a}$ & a \\
\hline Cs-137 & 97.73 & 0.17 & 106.66 & 4.14 & 108.94 & 4.22 \\
\hline Eu-152 & a & a & a & a & a & a \\
\hline Eu-154 & $\mathrm{b}$ & $\mathrm{b}$ & $\mathrm{b}$ & $\mathbf{b}$ & $b$ & $\mathbf{b}$ \\
\hline Eu-155 & $\mathbf{a}$ & $\mathbf{a}$ & $\mathbf{a}$ & $\mathbf{a}$ & $\mathrm{a}$ & $\mathrm{a}$ \\
\hline Pu-238 & -29.19 & 10.77 & $b$ & b & 129.19 & 10.77 \\
\hline $\mathrm{Pu}-239 / 240$ & 10.25 & 2.09 & $\mathrm{~b}$ & b & 89.75 & 2.09 \\
\hline Gross- $\alpha$ & -1.38 & 6.92 & 0.00 & 0.00 & 101.38 & 6.92 \\
\hline Gross- $\beta$ & 22.99 & 2.56 & 17.63 & 1.10 & 94.64 & 3.18 \\
\hline
\end{tabular}

Insufficient data for calculation; measured concentration in original sludge was below the detectable limit or was not measured.

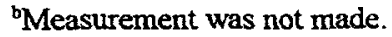


It is also consistent with the expected behavior of chromium in this system. Examination of results on aluminum show that, based on residual solids, $34.5 \% \pm 0.8 \%$ of it was removed while the analysis of the leachate and rinse shows a $7.6 \% \pm 0.2 \%$ removal. Recovery of $73.1 \% \pm 0.9 \%$ of the aluminum indicates only a fair material balance. Results on phosphorus are disappointing because analysis of the solid residue indicates $65.6 \% \pm 3.0 \%$ removal, while the analysis on the leachate and rinse solutions indicates $0 \%$ removal. This occurred because phosphorus was below the detectable limit in the process liquids and led to a calculated recovery of $34.4 \% \pm 3.0 \%$. One possible cause of the absence of phosphorus in the liquid is that it may have precipitated from solution between the time the solids and liquids were separated and the time that an aliquot was withdrawn for analysis. This is partly corroborated by the relatively low errors propagated to the calculated results. If the estimated errors in the analysis are taken as correct, the estimated errors in the calculated results are too small to account for the missing material. The possibility of precipitation led to a change in procedure wherein the entire amount of both phases, beginning with test \#5, was analyzed.

Poor material balances are not always indicative of a precipitation problem but could be an artifact of the small concentration of a given species in the original sludge. For example, the dilution factors were such that the lower limit of detection of thorium in the residue (Table 4.3) was greater than the concentration in the original sludge. The rules for computation, as already discussed, resulted in a value indicating complete removal of thorium from the sludge. However, based on the analysis of the process liquids, the percent removal could have ranged from 10.5 to $188 \%$. This uncertainty is reflected in the percent recovery, $99.3 \% \pm 88.8 \%$. Because thorium is insoluble in alkali solutions, a high percentage removal was not expected. Conflicts between measured and expected values could also result if the measured concentration of thorium in the original sludge were lower than the actual concentration. Suppose thorium exists in the sludge as thorium hypophosphate $\left(\operatorname{ThP}_{2} \mathrm{O}_{6} \cdot 11 \mathrm{H}_{2} \mathrm{O}\right)$, which is insoluble in either alkali or acidic solutions. The data on S-104 indicate that phosphorus, at 
$2480 \mu \mathrm{g} / \mathrm{g}$, is present in great excess compared with thorium, at $2.67 \mu \mathrm{g} / \mathrm{g}$. As an acid-insoluble form, the thorium could remain with the insoluble residue (assumed to be silicon dioxide) and fail to report for analysis by ICP-AES.

Generally the errors in the data and in the results are small for the more abundant constituents. Large errors occur where the analyte is near the detection level or where experimental difficulties, such as precipitation, may have occurred. Changing the experimental method to analyze the entire leachate solution, rather than a small aliquot, seems to have mitigated the latter problem. The reader may notice that the concentration of a given material is occasionally greater in the residue than in the original sludge, for example, uranium in Table 4.13. This means that other constituents were preferentially removed, and those that were not removed became a greater fraction of the total remaining mass.

To simplify the analysis of the effect of operational parameters on the leaching process, a subset of the more important sludge constituents was selected. Aluminum, chromium, and phosphorus were selected because these constituents strongly influence the quantities of glass that will be produced to immobilize the waste. Uranium and cesium were included to ascertain how these components partition between the solid and liquid phases, which has implications on the cleanup and recycle of the enhanced sludge washing solutions.

The operating conditions for each of the six tests on sludge S-104 and the percentage of selected constituents removed from the sludge are summarized in Table 4.15. Values of percentage removed are those based on the analysis of the residue. It was thought that these values would give the more consistent results because the untreated sludge and the sludge residue were analyzed using the same methodology. As shown in Table 4.15, chromium and cesium were readily removed from the sludge, with $>95 \%$ removal under all conditions tested. Comparing test $\# 1$ with test $\# 2$ indicates that aluminum removal increased with increased leaching time. Tests \#3 and \#4 show no increase in 
Table 4.15. Summary of effects of operating parameters on caustic leaching of S-104 sludge

\begin{tabular}{ccccccccccc}
\hline & $\begin{array}{c}\text { Liquid: } \\
\text { solids }\end{array}$ & $\mathrm{NaOH}$ & $\mathrm{T}$ & $\mathrm{Time}$ & \multicolumn{5}{c}{ \% Removal } \\
\cline { 7 - 10 } Test & $(\mathrm{mL} / \mathrm{g})$ & $(M)$ & $\left({ }^{\circ} \mathrm{C}\right)$ & $(\mathrm{h})$ & $\mathrm{Al}$ & $\mathrm{Cr}$ & $\mathrm{P}$ & $\mathrm{U}$ & $\mathrm{Cs}-137$ \\
\hline 1 & 9 & 3.8 & 67 & 4 & 34.5 & 98.0 & 65.6 & 37.2 & 97.9 \\
2 & 9 & 3.8 & 67 & 24 & 50.3 & 98.5 & 72.8 & 46.1 & 98.0 \\
3 & 6 & 3.99 & 70 & 21 & 20.4 & 97.4 & 44.3 & 15.6 & 95.0 \\
4 & 12 & 3.99 & 70 & 21 & 20.8 & 97.6 & 42.5 & 12.1 & 96.5 \\
5 & 12 & 6.33 & 70 & 21 & 26.6 & 97.1 & 48.9 & 7.60 & 96.9 \\
9 & 32 & 6.33 & 80 & 126 & 96.3 & 99.1 & 96.3 & 3.08 & 97.7 \\
\hline
\end{tabular}


aluminum removal with increased liquid:solids ratio. Increasing the caustic concentration in test \#5 slightly increased the amount of aluminum removed compared with test \#4. In test \#9, where all four variables of liquid:solids ratio, caustic concentration, temperature, and time were largest, a great increase in the percentage of aluminum removed was observed. To visualize these effects, the data in Table 4.15 were smoothed using an inverse distance method (Ulrich et al., 1995) to generate threedimensional mesh plots. Fig. 4.1 illustrates the effect of caustic concentration and temperature without regard to the other parameters. The caustic concentration, in the range between 3.8 and $6.4 \mathrm{M}$, has little effect on the percentage of aluminum removed from the sludge. However, the temperature is shown to have a large effect. In Fig. 4.2, the percentage of aluminum removed from the sludge is plotted as a function of liquid:solids ratio and temperature, without regard to the other two parameters. This plot shows that both liquid:solids ratio and temperature strongly affect the percentage of aluminum removed from the sludge. One must bear in mind that these plots do not model physical chemistry but are merely convenient for showing trends. There is the temptation to conclude that aluminum removal is solubility limited. However, the test done at both the high temperature and high liquid:solids ratio also happens to be the test having the long leaching time $(126 \mathrm{~h})$, perhaps indicating * that the process is reaction rate limited.

Removal of phosphorus seemed to coincide with that of aluminum. Plotting the percentage of phosphorus removed as a function of the percentage of aluminum removed, as shown in Fig. 4.3, illustrates the trend. Removal of uranium seems to vary inversely with caustic concentration, removal being the highest at the lowest caustic concentration (3.8M) tested.

Sludge S-104 has also been studied by Rapko et al. (1995) and Lumetta et al. (1997b). The concentrations of selected components measured in dry, untreated sludge in those studies are compared with the present study in Table 4.16 . The analyses agree to within about $25 \%$ on aluminum, chromium, uranium, cesium-137, and plutonium-239/240. However, there is a great discrepancy in 


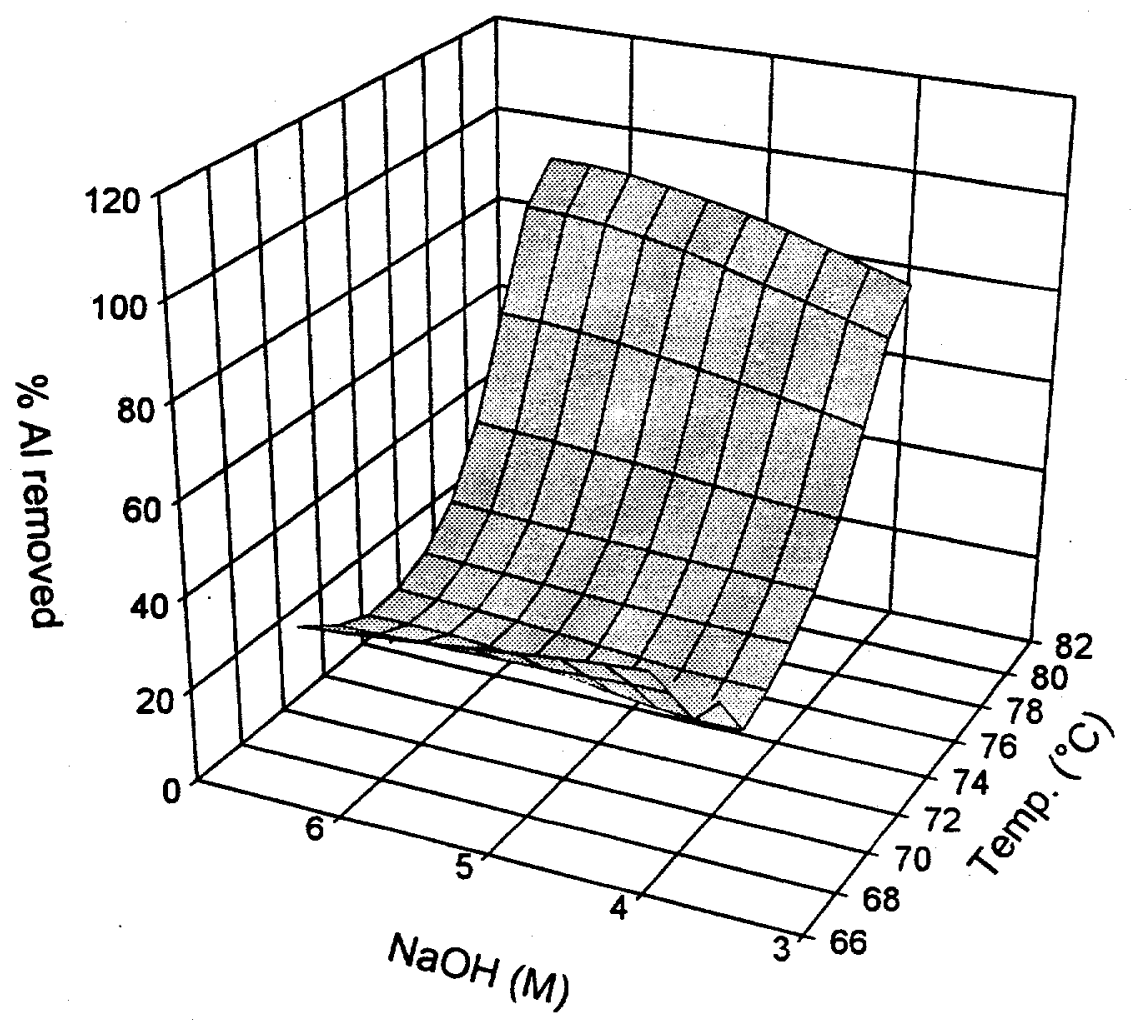

Fig. 4.1. The percentage of aluminum removed from S-104 sludge as a function of temperature and caustic concentration. 


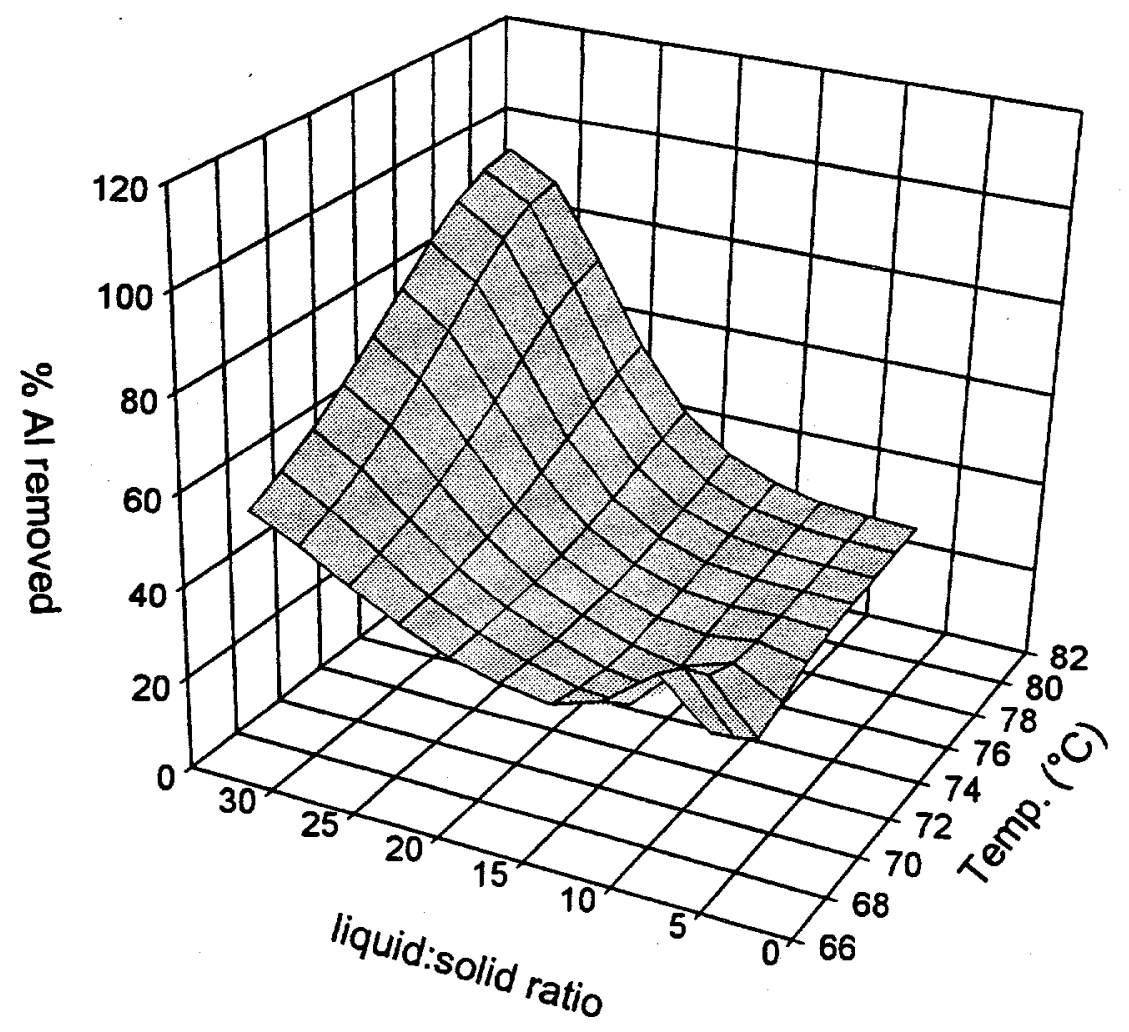
Fig. 4.2. The percentage of aluminum removed from S-104 sludge as a function of
temperature and liquid-to-solids ratio. 


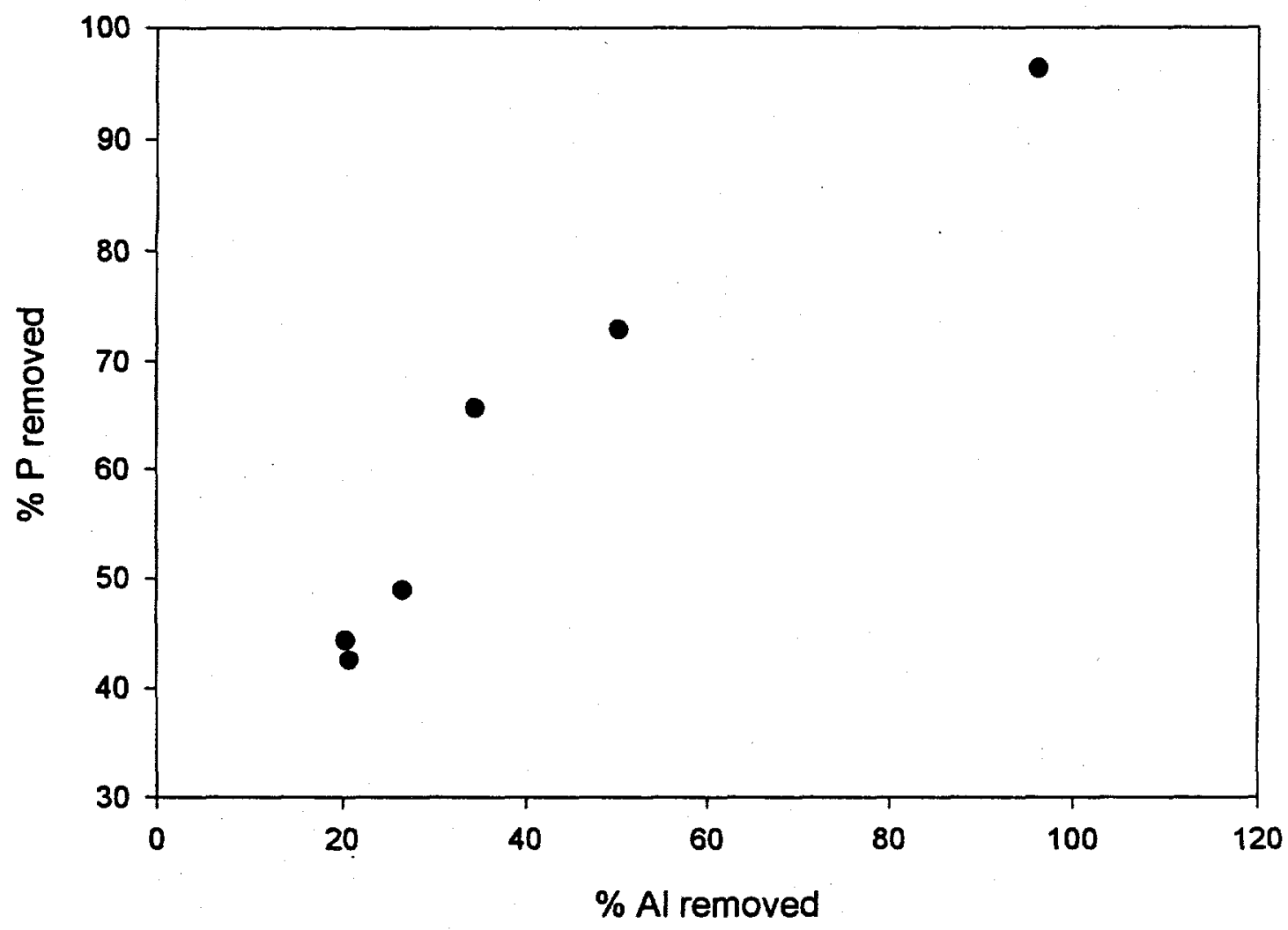

Fig. 4.3. Phosphorus follows aluminum removal for S-104 sludge. 
the results for phosphorus. The amount of each of these materials removed from the sludge by the different researchers is compared in Table 4.17. Chromium and cesium were readily removed from the sludge under all process conditions evaluated. Removal of aluminum varied appreciably; as little as $20 \%$ was removed in $21 \mathrm{~h}$ at $70^{\circ} \mathrm{C}$ using $4 \mathrm{MNaOH}$, but nearly all was removed in $75 \mathrm{~h}$ when the temperature was increased to $80^{\circ} \mathrm{C}$ or more at sodium hydroxide concentrations between 3 and $6 \mathrm{M}$. Removal of uranium and plutonium was higher than expected, which is a consequence of having selected the analysis of the residue for computing percentage removal. Returning to the data tables, the analysis of the leachate solution indicates that neither uranium nor plutonium were removed from the sludge by caustic leaching. This is consistent with the known solubility of uranium and plutonium in caustic solutions. Except for tests $\# 1$ and \#2, the values associated with gross-alpha analysis confirm that plutonium was not removed.

\subsection{SLUDGE SX-113}

Two tests were performed on sludge samples from tank $S X-113$. Analyses of this sludge, as shown in Table 4.18, indicate that it has relatively low aluminum, chromium, and phosphorus content. However, iron and silicon are quite abundant. Analytical data describing the samples taken from the two tests are given in. Tables 4.18 and 4.20 , and the associated calculated results are given in Tables 4.19 and 4.21 , respectively.

Table 4.22 summarizes the effect of the two different leaching conditions on the removal of aluminum, chromium, phosphorus, uranium, and cesium from sludge SX-113. At a fixed sodium hydroxide concentration, a combined increase in the liquid:solids ratio, leaching time, and leaching temperature increased the percentage of constituents leached from the sludge. The recovery of chromium was low, 53 to $66 \%$, but there was only a small amount of it in the sludge initially. The high phosphorus removal (92\%) in test \#10 was probably not real since the recovery was only $11 \%$. A large fraction of the uranium was removed, but there was only a small quantity of it in the sludge 
Table 4.16. Concentration of selected constituents in dry, untreated S-104 sludge determined by different researchers

\begin{tabular}{ccccc}
\hline Species & Unit of measure & PNNL $^{\mathrm{a}}$ & PNNL $^{b}$ & ORNL $^{\mathrm{c}}$ \\
\hline $\mathrm{Al}$ & $\mu \mathrm{g} / \mathrm{g}$ & 150000 & 153000 & 166000. \\
$\mathrm{Cr}$ & $\mu \mathrm{g} / \mathrm{g}$ & 4700. & 4470 & 3670. \\
$\mathrm{P}$ & $\mu \mathrm{g} / \mathrm{g}$ & $<200$. & 17 & 2940. \\
$\mathrm{U}$ & $\mu \mathrm{g} / \mathrm{g}$ & 10100. & 9360. & 9950. \\
$\mathrm{Cs}-137$ & $\mu \mathrm{Ci} / \mathrm{g}$ & 91.6 & & 86.5 \\
$\mathrm{Pu}-239 / 240$ & $\mu \mathrm{Ci} / \mathrm{g}$ & 0.545 & & 0.628 \\
\hline
\end{tabular}

${ }^{2}$ Rapko et al., PNL-10712 (1995).

bLumetta et al., PNNL-1 1636 (1997b).

This work.

Table 4.17. Comparison of percentage of selected species removed from S-104 sludge by different researchers

\begin{tabular}{ccccc}
\hline & \multicolumn{3}{c}{ \% Removed } \\
\cline { 2 - 5 } Species & PNNL $^{\mathrm{a}}$ & PNNL $^{\mathrm{b}}$ & ORNL $^{\mathrm{c}}$ & ORNL $^{\mathrm{d}}$ \\
$\mathrm{Al}$ & 38 & 99 & 21 & 96 \\
$\mathrm{Cr}$ & 97 & 99 & 97 & 99 \\
$\mathrm{P}$ & & & 44 & 96 \\
$\mathrm{U}$ & 0 & 0 & 12 & 3 \\
$\mathrm{Cs}$ & 98 & & 97 & 98 \\
$\mathrm{Pu}$ & & & 6 & 10 \\
\hline
\end{tabular}

${ }^{8}$ Rapko et al., PNL-10712 (1995); $10 \mathrm{MNaOH}$ for $5 \mathrm{~h}, 3 \mathrm{MNaOH}$ for $5 \mathrm{~h}, 100^{\circ} \mathrm{C}$, $1: 1 \leq$ liquid:solid $\leq 2: 1$.

${ }^{b}$ Lumetta et al., PNNL-11636 (1997b); $3 \mathrm{MNaOH}, 75 \mathrm{~h}, 100^{\circ} \mathrm{C}$, liquid:solids of 10:1.

'This work; $4 \mathrm{M} \mathrm{NaOH}, 21 \mathrm{~h}, 70^{\circ} \mathrm{C}$, and liquid:solids of $12: 1$.

${ }^{\mathrm{d}}$ This work; $6 \mathrm{MNaOH}, 126 \mathrm{~h}, 80^{\circ} \mathrm{C}$, and liquid:solids of $32: 1$. 
Table 4.18. Test \#6 - concentrations of species in enhanced sludge washing process steps for SX-113 sludge (leach conditions: liquid:solids, $20 \mathrm{~mL} / \mathrm{g}$; $\mathrm{NaOH}, 6.33 \mathrm{M}$; temperature, $70^{\circ} \mathrm{C}$; time, $21 \mathrm{~h}$ )

\begin{tabular}{|c|c|c|c|c|c|c|c|c|}
\hline \multirow[b]{2}{*}{ Species } & \multicolumn{2}{|c|}{$\begin{array}{c}\text { Original sludge } \\
(\mu \mathrm{g} / \mathrm{g})\end{array}$} & \multicolumn{2}{|c|}{$\begin{array}{l}\text { Residual } \\
(\mu \mathrm{g} / \mathrm{g})\end{array}$} & \multicolumn{2}{|c|}{$\begin{array}{l}\text { Leachate } \\
(\mu \mathrm{g} / \mathrm{g})\end{array}$} & \multicolumn{2}{|c|}{$\begin{array}{l}\text { Rinse } \\
(\mu \mathrm{g} / \mathrm{g})\end{array}$} \\
\hline & Value & Std deviation & Value & Std deviation & Value & Std deviation & Value & Std deviation \\
\hline $\mathrm{Ag}$ & $<2.44^{\mathrm{a}}$ & $\mathrm{b}$ & $<4.85$ & 0.429 & $<0.409$ & $\mathrm{~b}$ & $<0.417$ & $b$ \\
\hline Al & 7320 & 52.7 & 5080 & 191 & 307 & 11.2 & 10 & 0.209 \\
\hline $\mathrm{Ba}$ & 20.5 & 0.244 & 33.7 & 0 & $<0.0409$ & $\mathrm{~b}$ & $<0.0417$ & $b$ \\
\hline $\mathrm{Be}$ & $<0.122$ & $\mathrm{~b}$ & $<0.243$ & $b$ & $<0.0205$ & b & $<0.0209$ & b \\
\hline $\mathrm{Bi}$ & 60.9 & 10.5 & $<97.1$ & 3.65 & $<8.18$ & $\mathrm{~b}$ & $<8.34$ & $\mathrm{~b}$ \\
\hline $\mathrm{Ca}$ & 1770 & 11.7 & 1830 & 0 & 1.37 & 0.0818 & 0.876 & 0.0417 \\
\hline $\mathrm{Cd}$ & $<2.44$ & $b$ & $<4.85$ & b & $<0.409$ & $\mathrm{~b}$ & $<0.417$ & $\mathrm{~b}$ \\
\hline Co & $<2.32$ & $b$ & $<4.61$ & 3 & $<0.389$ & b & $<0.396$ & b \\
\hline $\mathrm{Cr}$ & 57.1 & 0.488 & 38.1 & 0.644 & 0.716 & 0.0818 & $<0.104$ & b \\
\hline $\mathrm{Cu}$ & 13.4 & 0.732 & 18.2 & 49.6 & 9.9 & 3.89 & 1.94 & 0.167 \\
\hline $\mathrm{Fe}$ & 10400 & 26.4 & 13100 & 9.44 & 91.4 & 0.0818 & 1.69 & 0.125 \\
\hline $\mathrm{K}$ & 85.9 & 1.95 & 42 & 0 & 22 & 1.39 & 3.75 & 1.67 \\
\hline $\mathrm{Mg}$ & 1580 & 20.3 & 2330 & 3 & $<0.511$ & $\mathrm{~b}$ & $<0.521$ & $\mathbf{b}$ \\
\hline $\mathrm{Mn}$ & 234 & 1.22 & 325 & 5.58 & 0.777 & 0.245 & $<0.0209$ & b \\
\hline $\mathrm{Ni}$ & 5.12 & 1.22 & 15.3 & 1.72 & $<0.409$ & $b$ & $<0.417$ & $\mathrm{~b}$ \\
\hline $\mathrm{P}$ & 3890 & 39.3 & 4820 & 19.7 & 33.3 & 4.99 & $<2.09$ & b \\
\hline $\mathrm{Sb}$ & $<24.4$ & b & $<48.5$ & $\mathrm{~b}$ & $<4.09$ & $\mathrm{~b}$ & $<4.17$ & b \\
\hline $\mathrm{Si}$ & 220000 & 0 & 22000 & 0 & 488 & 2.7 & 425 & 1.17 \\
\hline $\mathrm{Sr}$ & 15.3 & 0 & 21.1 & 2.36 & $<0.102$ & $\mathrm{~b}$ & $<0.104$ & $\mathrm{~b}$ \\
\hline Th & 10.9 & 4.64 & $<12.1$ & 4.08 & $<1.02$ & $\mathrm{~b}$ & $<1.04$ & $b$ \\
\hline $\mathrm{U}$ & 94.6 & 13.9 & $<24.3$ & 59 & 5.89 & 1.43 & $<2.09$ & b \\
\hline V & 123 & 1.22 & 30.8 & 0 & 7.98 & 0.0409 & 0.25 & 0.0417 \\
\hline $\mathrm{Zn}$ & 218 & 2.93 . & 36.9 & 7.94 & 4.23 & 0.818 & $<0.626$ & $\mathrm{~b}$ \\
\hline Radiospecies & \multicolumn{2}{|c|}{$\mathrm{Ba} / \mathrm{g}$} & \multicolumn{2}{|r|}{$\mathrm{Ba} / \mathrm{g}$} & \multicolumn{2}{|c|}{$\mathrm{Ba} / \mathrm{g}$} & \multicolumn{2}{|c|}{$\mathrm{Ba} / \mathrm{g}$} \\
\hline Co-60 & $<42$ & $\mathrm{~b}$ & 34 & 30 & $<1$ & $b$ & $<1$ & $\mathrm{~b}$ \\
\hline Cs- 137 & 840000 & 10000 & 480000 & 1000 & 38000 & 1000 & 2400 & 100 \\
\hline $\mathrm{Eu}-152$ & $<120$ & b & $<70$ & $\mathrm{~b}$ & $<6$ & b & $<4$ & b \\
\hline Eu-154 & $<260$ & $\mathrm{~b}$ & 340 & 200 & $<8$ & b & $<3$ & $b$ \\
\hline Eu-155 & $<650$ & b & $<540$ & $b$ & $<37$ & $\mathbf{b}$ & $<7$ & $b$ \\
\hline Pu-238 & 70 & 4 & 40 & 10 & 1 & 0.3 & c & c \\
\hline $\mathrm{Pu}-239 / 240$ & 2300 & 200 & 2000 & 300 & 31 & 8 & c & c \\
\hline Pu-all & 2400 & 200 & 2000 & 300 & 32 & 8 & 0.3 & 1.1 \\
\hline Gross- $\alpha$ & 2700 & 300 & 2600 & 2000 & 33 & 14 & 0.28 & 0.56 \\
\hline Gross- $\beta$ & 3000000 & 100000 & 3200000 & 1000000 & 46000 & 1000 & 3000 & 100 \\
\hline
\end{tabular}
value.

The symbol < indicates that the concentration was below the detectable limit given by the following numerical

No standard deviation for values below the detectable limit.

Not measured. 
Table 4.19. Test \#6 - percentage of each species solubilized and percentage recovery for SX-113 sludge (leach conditions: liquid:solids, $20 \mathrm{~mL} / \mathrm{g} ; \mathrm{NaOH}, 6.33 \mathrm{M}$; temperature, $70^{\circ} \mathrm{C}$; time, $21 \mathrm{~h}$ )

\begin{tabular}{|c|c|c|c|c|c|c|}
\hline \multirow[b]{2}{*}{ Species } & \multicolumn{2}{|c|}{$\begin{array}{c}\text { Based on residue } \\
\qquad(\%)\end{array}$} & \multicolumn{2}{|c|}{$\begin{array}{c}\text { Based on leachate \& rinse } \\
(\%) \\
\end{array}$} & \multicolumn{2}{|c|}{$\begin{array}{l}\text { Recovery } \\
(\%) \\
\end{array}$} \\
\hline & Value & Std deviation & Value & Std deviation & Value & Std deviation \\
\hline $\mathrm{Ag}$ & $\mathbf{a}$ & $\mathrm{a}$ & a & a & $\mathbf{a}$ & a \\
\hline $\mathrm{Al}$ & 51.04 & 1.87 & 62.12 & 2.16 & 111.08 & 2.91 \\
\hline $\mathrm{Ba}$ & -15.97 & 1.38 & 0.00 & 0.00 & 115.97 & 1.38 \\
\hline $\mathrm{Be}$ & $\mathbf{a}$ & $\mathrm{a}$ & $\mathrm{a}$ & a & $\mathrm{a}$ & $\mathrm{a}$ \\
\hline $\mathrm{Bi}$ & 100.00 & 4.23 & 0.00 & 0.00 & 0.00 & 4.23 \\
\hline $\mathrm{Ca}$ & 27.06 & 0.48 & 2.60 & 0.10 & 75.54 & 0.51 \\
\hline $\mathrm{Cd}$ & $\mathbf{a}$ & a & $\mathbf{a}$ & a & a & $\mathrm{a}$ \\
\hline $\mathrm{Co}$ & a & a & a. & $\mathbf{a}$ & a & a \\
\hline $\mathrm{Cr}$ & 52.93 & 0.89 & 17.31 & 1.98 & 64.38 & 2.20 \\
\hline $\mathrm{Cu}$ & 4.18 & 261.19 & 1468.26 & 410.42 & 1564.08 & 487.34 \\
\hline $\mathrm{Fe}$ & 11.14 & 0.23 & 12.63 & 0.05 & 101.50 & 0.27 \\
\hline $\mathrm{K}$ & 65.51 & 0.78 & 488.74 & 65.20 & 523.24 & 65.34 \\
\hline $\mathrm{Mg}$ & -4.04 & 1.34 & 0.00 & 0.00 & 104.04 & 1.34 \\
\hline $\mathrm{Mn}$ & 2.02 & 1.76 & 4.58 & 1.45 & 102.57 & 2.28 \\
\hline Ni & -110.82 & 55.54 & 0.00 & 0.00 & 210.82 & 55.54 \\
\hline $\mathbf{P}$ & 12.59 & 0.95 & 11.81 & 1.77 & 99.23 & 2.07 \\
\hline $\mathrm{Sb}$ & $\mathbf{a}$ & a & $a$ & a & $\mathbf{a}$ & a \\
\hline $\mathrm{Si}$ & 92.95 & 0.00 & 9.05 & 0.02 & 16.10 & 0.02 \\
\hline $\mathrm{Sr}$ & 2.71 & 10.88 & 0.00 & 0.00 & 97.29 & 10.88 \\
\hline Th & 100.00 & 26.41 & 0.00 & 0.00 & 0.00 & 26.41 \\
\hline $\mathrm{U}$ & 100.00 & 44.00 & 85.93 & 24.39 & 85.93 & 50.31 \\
\hline V & 82.33 & 0.18 & 95.84 & 1.49 & 113.50 & 1.61 \\
\hline $\mathrm{Zn}$ & 88.06 & 2.57 & 26.78 & 5.19 & 38.72 & 5.80 \\
\hline \multicolumn{7}{|l|}{ Radiospecies } \\
\hline $\mathrm{Co}-60$ & a & $\mathbf{a}$ & $\mathbf{a}$ & $\mathbf{a}$ & $\mathbf{a}$ & $\mathbf{a}$ \\
\hline Cs-137 & 59.69 & 0.49 & 71.29 & 1.89 & 111.60 & 2.15 \\
\hline Eu-152 & a & $\mathbf{a}$ & $\mathbf{a}$ & $\mathbf{a}$ & $\mathbf{a}$ & a \\
\hline Eu-154 & $\mathbf{a}$ & $\mathbf{a}$ & $\mathbf{a}$ & $\mathbf{a}$ & a & a \\
\hline Eu-155 & $\mathbf{a}$ & $\mathbf{a}$ & $\mathbf{a}$ & $\mathbf{a}$ & $\mathbf{a}$ & $\mathbf{a}$ \\
\hline Pu-238 & 59.69 & 10.34 & 19.72 & 6.02 & 60.03 & 12.18 \\
\hline Pu-239/240 & 38.65 & 10.64 & 18.60 & 5.07 & 79.95 & 12.49 \\
\hline Pu-all & 41.21 & 10.09 & 18.79 & 5.06 & 77.58 & 11.95 \\
\hline Gross- $\alpha$ & 32.06 & 52.80 & 17.19 & 7.43 & 85.12 & 53.59 \\
\hline Gross- $\beta$ & 24.75 & 23.65 & 24.26 & 0.94 & 99.51 & 23.75 \\
\hline
\end{tabular}

"Insufficient data for calculation; measured concentration in original sludge was below the detectable limit or was not measured. 
Table 4.20. Test \#10 - concentrations of species in enhanced sludge washing process steps for $\mathrm{SX}-113$ sludge (leach conditions: liquid:solids, $44 \mathrm{~mL} / \mathrm{g} ; \mathrm{NaOH}, 6.33 \mathrm{M}$; temperature, $80^{\circ} \mathrm{C}$; time, $126 \mathrm{~h}$ )

\begin{tabular}{|c|c|c|c|c|c|c|c|c|}
\hline \multirow[b]{2}{*}{ Species } & \multicolumn{2}{|c|}{$\begin{array}{c}\text { Original sludge } \\
(\mu \mathrm{g} / \mathrm{g})\end{array}$} & \multicolumn{2}{|c|}{$\begin{array}{c}\text { Residual } \\
(\mu \mathrm{g} / \mathrm{g})\end{array}$} & \multicolumn{2}{|c|}{$\begin{array}{l}\text { Leachate } \\
(\mu \mathrm{g} / \mathrm{g})\end{array}$} & \multicolumn{2}{|c|}{$\begin{array}{l}\text { Rinse } \\
(\mu g / g)\end{array}$} \\
\hline & Value & Std deviation & Value & Std deviation & Value & Std deviation & Value & Std deviation \\
\hline $\mathrm{Ag}$ & $<2.44^{\mathrm{a}}$ & b & $<1.36$ & b & $<0.0844$ & $b$ & $<0.0986$ & b \\
\hline $\mathrm{Al}$ & 7320 & 52.7 & 2820 & 48.6 & 215 & 11.9 & 6.61 & 0.661 \\
\hline $\mathrm{Ba}$ & 20.5 & 0.244 & 37.1 & 0.136 & 0.0169 & 0 & $<0.00986$ & $\mathrm{~b}$ \\
\hline $\mathrm{Be}$ & $<0.122$ & $\mathrm{~b}$ & $<0.068$ & b & $<0.00422$ & $\mathrm{~b}$ & $<0.00493$ & b \\
\hline $\mathrm{Bi}$ & 60.9 & 10.5 & $<1.02$ & b & $<0.0633$ & b & $<0.0247$ & b \\
\hline $\mathrm{Ca}$ & 1770 & 11.7 & 2470 & 14.3 & 0.477 & 0.0338 & 0.463 & 0.463 \\
\hline $\mathrm{Cd}$ & $<2.44$ & $b$ & $<1.36$ & $\mathbf{b}$ & $<0.0844$ & b & $<0.0986$ & $\mathrm{~b}$ \\
\hline Co & $<2.32$ & $\mathrm{~b}$ & 1.63 & 0.952 & $<0.0802$ & b & $<0.0937$ & $\mathrm{~b}$ \\
\hline $\mathrm{Cr}$ & 57.1 & 0.488 & 35.6 & 0.136 & 0.397 & 0.0169 & 0.0394 & 0.00986 \\
\hline $\mathrm{Cu}$ & 13.4 & 0.732 & 5.3 & 0.272 & 0.228 & 0.00844 & 0.296 & 0.0296 \\
\hline $\mathrm{Fe}$ & 10400 & 26.4 & 13400 & 14.3 & 66.9 & 0.852 & 0.478 & 0.0478 \\
\hline $\mathbf{K}$ & 85.9 & 1.95 & 44.5 & 0.68 & 127 & 7.97 & 1.14 & 0.237 \\
\hline $\mathrm{Mg}$ & 1580 & 20.3 & 2610 & 25.7 & 0.122 & 0.118 & $<0.123$ & $\mathrm{~b}$ \\
\hline $\mathrm{Mn}$ & 234 & 1.22 & 289 & 0.544 & 0.57 & 0.00844 & $<0.00493$ & b \\
\hline $\mathrm{Ni}$ & 5.12 & 1.22 & 9.04 & 0.816 & $<0.0844$ & $\mathrm{~b}$ & $<0.0986$ & b \\
\hline$P$ & 3890 & 39.3 & 581 & 36.4 & 4.04 & 0.996 & $<0.493$ & $b$ \\
\hline $\mathrm{Sb}$ & $<24.4$ & $\mathrm{~b}$ & 59.4 & 85 & $<0.844$ & $\mathrm{~b}$ & $<0.0247$ & $\mathrm{~b}$ \\
\hline $\mathrm{Si}$ & 220000 & 0 & 18000 & 0 & c & c & c & c \\
\hline $\mathrm{Sr}$ & 15.3 & 0 & 21.5 & 0.408 & 0.0422 & 0 & $<0.0247$ & $\mathrm{~b}$ \\
\hline Th & 10.9 & 4.64 & $<3.4$ & $\mathrm{~b}$ & $<0.211$ & b & $<0.247$ & b \\
\hline $\mathrm{U}$ & 94.6 & 13.9 & 11.8 & 4.22 & 2.27 & 0.0338 & $<0.493$ & b \\
\hline V & 123 & 1.22 & 7.62 & 0.136 & 4 & 0.0253 & 0.163 & 0.0163 \\
\hline $\mathrm{Zn}$ & 218 & 2.93 & 31.1 & 1.9 & 1.61 & 0.203 & $<0.148$ & b \\
\hline Radiospecies & & $g / g$ & & $\mathrm{Bg} / \mathrm{g}$ & & $\mathrm{Bq} / \mathrm{g}$ & & $3 \mathrm{~g} / \mathrm{g}$ \\
\hline Co-60 & $<42$ & b & & & 7.5 & 0 & 15.3 & 0 \\
\hline Cs-137 & 840000 & 10000 & 210000 & 10000 & 37000 & 1000 & 870 & 50 \\
\hline Eu-152 & $<120$ & $\mathrm{~b}$ & c & c & $<170$ & b & $<52$ & b \\
\hline Eu-154 & $<260$ & $\mathrm{~b}$ & c & c & $<150$ & $b$ & $<46$ & b \\
\hline Eu-155 & $<650$ & $b$ & c & $\mathrm{c}$ & $<270$ & $\mathrm{~b}$ & $<32$ & b \\
\hline Pu-238 & 70 & 4 & 100 & 10 & c & c & c & c \\
\hline $\mathrm{Pu}-239 / 240$ & 2300 & 200 & 2300 & 100 & c & c & c & c \\
\hline Pu-all & 2400 & 200 & c & $\mathrm{c}$ & $\mathrm{c}$ & c & c & c \\
\hline Gross- $\alpha$ & 2700 & 300 & 2900 & 500 & 27 & 13 & c & c \\
\hline Gross- $\beta$ & 3000000 & 100000 & 2900000 & 100000 & 32000 & 1000 & 1100 & 100 \\
\hline
\end{tabular}

The symbol < indicates that the concentration was below the detectable limit given by the following numerical value.

'No standard deviation for values below the detectable limit.

Not measured. 
Table 4.21. Test \#10 - percentage of each species solubilized and percentage recovery for SX-113 sludge (leach conditions: liquid:solids, $44 \mathrm{~mL} / \mathrm{g} ; \mathrm{NaOH}, 6.33 \mathrm{M}$; temperature, $80^{\circ} \mathrm{C}$; time, $126 \mathrm{~h}$ )

\begin{tabular}{|c|c|c|c|c|c|c|}
\hline \multirow[b]{2}{*}{ Species } & \multicolumn{2}{|c|}{$\begin{array}{c}\text { Based on residue } \\
(\%) \\
\end{array}$} & \multicolumn{2}{|c|}{$\begin{array}{c}\text { Based on leachate \& rinse } \\
(\%) \\
\end{array}$} & \multicolumn{2}{|c|}{$\begin{array}{c}\text { Recovery } \\
(\%) \\
\end{array}$} \\
\hline & Value & Std deviation & Value & Std deviation & Value & Std deviation \\
\hline $\mathrm{Ag}$ & a & $\mathrm{a}$ & $\mathbf{a}$ & $\mathrm{a}$ & $\mathrm{a}$ & $\mathrm{a}$ \\
\hline $\mathrm{Al}$ & 78.69 & 0.40 & 81.75 & 4.40 & 103.06 & 4.44 \\
\hline $\mathrm{Ba}$ & -0.08 & 1.25 & 2.21 & 0.03 & 102.29 & 1.27 \\
\hline $\mathrm{Be}$ & $\mathbf{a}$ & $\mathrm{a}$ & $\mathbf{a}$ & $\mathrm{a}$ & a & $\mathrm{a}$ \\
\hline $\mathrm{Bi}$ & 100.00 & 0.00 & 0.00 & 0.00 & 0.00 & 0.00 \\
\hline $\mathrm{Ca}$ & 22.83 & 0.68 & 1.62 & 0.90 & 78.80 & 1.14 \\
\hline $\mathrm{Cd}$ & $\mathbf{a}$ & $\mathrm{a}$ & $\mathrm{a}$ & $\mathrm{a}$ & $a$ & a \\
\hline Co & $\mathbf{a}$ & 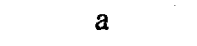 & $\mathrm{a}$ & a & $\mathbf{a}$ & $\mathbf{a}$ \\
\hline $\mathrm{Cr}$ & 65.52 & 0.32 & 21.00 & 1.01 & 55.48 & 1.11 \\
\hline $\mathrm{Cu}$ & 78.13 & 1.64 & 121.85 & 10.26 & 143.72 & 11.13 \\
\hline $\mathrm{Fe}$ & 28.74 & 0.20 & 17.38 & 0.22 & 88.64 & 0.32 \\
\hline $\mathrm{K}$ & 71.35 & 0.78 & 4004.09 & 264.68 & 4032.74 & 264.91 \\
\hline $\mathrm{Mg}$ & 8.64 & 1.48 & 0.21 & 0.20 & 91.56 & 1.49 \\
\hline $\mathrm{Mn}$ & 31.70 & 0.38 & 6.52 & 0.10 & 74.82 & 0.42 \\
\hline $\mathrm{Ni}$ & 2.36 & 24.88 & 0.00 & 0.00 & 97.64 & 24.88 \\
\hline $\mathbf{P}$ & 91.74 & 0.52 & 2.78 & 0.69 & 11.04 & 0.87 \\
\hline $\mathrm{Sb}$ & $\mathbf{a}$ & $\mathrm{a}$ & a & $\mathrm{a}$ & $\mathrm{a}$ & $\mathbf{a}$ \\
\hline $\mathrm{Si}$ & 95.48 & 0.00 & 0.00 & 0.00 & 4.52 & 0.00 \\
\hline $\mathrm{Sr}$ & 22.29 & 1.47 & 7.38 & 0.00 & 85.10 & 1.47 \\
\hline Th & 100.00 & 0.00 & 0.00 & 0.00 & 0.00 & 0.00 \\
\hline $\mathrm{U}$ & 93.10 & 2.67 & 64.24 & 9.49 & 71.14 & 10.78 \\
\hline V & 96.57 & 0.07 & 91.64 & 1.16 & 95.07 & 1.19 \\
\hline $\mathrm{Zn}$ & 92.11 & 0.49 & 19.77 & 2.51 & 27.66 & 2.57 \\
\hline \multicolumn{7}{|c|}{ Radiospecies } \\
\hline $\mathrm{Ca}-60$ & $\mathbf{a}$ & $\mathbf{a}$ & $\mathbf{a}$ & $\mathbf{a}$ & $\mathbf{a}$ & $\mathbf{a}$ \\
\hline Cs-137 & 86.17 & 0.68 & 121.50 & 3.51 & 135.33 & 3.64 \\
\hline Eu-152 & $\mathbf{a}$ & a & a & a & $\mathbf{a}$ & $\mathbf{a}$ \\
\hline Eu-154 & a & $\mathbf{a}$ & $\mathbf{a}$ & $\mathrm{a}$ & a & a \\
\hline Eu-155 & $\mathbf{a}$ & a & $\mathbf{a}$ & $\mathbf{a}$ & $\mathbf{a}$ & a \\
\hline $\mathrm{Pu}-238$ & 21.00 & 9.10 & $\mathrm{~b}$ & b & 79.00 & 9.10 \\
\hline $\mathrm{Pu}-239 / 240$ & 44.70 & 5.38 & $b$ & b & 55.30 & 5.38 \\
\hline Pu-all & $\mathbf{b}$ & $\mathbf{b}$ & b & $\mathbf{b}$ & $0: 00$ & 0.00 \\
\hline Gross- $\alpha$ & 40.60 & 12.18 & 26.77 & 13.23 & 86.17 & 19.05 \\
\hline Gross- $\boldsymbol{\beta}$ & 46.54 & 2.56 & 29.82 & 1.34 & 83.28 & 3.45 \\
\hline
\end{tabular}

'Insufficient data for calculation; measured concentration in original sludge was below the detectable limit or was not measured.

${ }^{b}$ Measurement was not made. 
initially. Uranyl hydroxide $\left[\mathrm{UO}_{2}(\mathrm{OH})_{2}\right]$ is known to be soluble in alkali carbonate solutions. If the sludge contained a significant concentration of, for instance, sodium carbonate, the uranium could be solubilized when the caustic leaching solution was added. Alternatively, if the sludge contained uranyl sodium carbonate $\left(\mathrm{UO}_{2} \mathrm{CO}_{3} \cdot 2 \mathrm{NaCO}_{3}\right)$, which is slightly soluble, it would enter solution when exposed to the relatively large volume of aqueous caustic solution. Thorium and bismuth were apparently removed from the sludge as indicated in Tables 4.19 and 4.21, but this is an artifact of the lower limits of detection in both the residue and leachate. About half of the plutonium was also removed from the sludge, which appears to be confirmed by the gross-alpha measurement.

Concentrations of selected species in the untreated sludge measured by Temer and Villarreal (1997) are compared to the present analysis in Table 4.23. The widest variation occurs in the determination of phosphorus. Analysis of the aluminum content varied by $50 \%$, but results for chromium, uranium, cesium-137, and plutonium-239/240 were comparable. Table 4.24 presents the percentage of each species, from the same subset, removed from the sludge under various conditions. Except for chromium and plutonium, our experiment at $80^{\circ} \mathrm{C}$ and $\sim 6 \mathrm{MNaOH}$ and the tests performed by Temer and Villarreal (1997) at $100^{\circ} \mathrm{C}$ and $\sim 3 \mathrm{MNaOH}$ removed similar percentages of the sludge components.

\subsection{SLUDGE C-105}

One test was performed with sludge $\mathrm{C}-105$ in which the material was leached with $6.33 \mathrm{M}$ $\mathrm{NaOH}$ at $70^{\circ} \mathrm{C}$ for $22 \mathrm{~h}$. Results of the analysis of the sludge and each "phase" into which the sludge was partitioned are given in Table 4.25. As shown in the table, the relative concentrations of aluminum, silicon, uranium, and plutonium are high in this sludge. The concentrations of iron and phosphorus are low. The percentages of each species removed from the sludge were calculated from the analytical data and are given in Table 4.26 . Approximately $97 \%$ of the aluminum, $71 \%$ of the 
Table 4.22. Effect of operating parameters on caustic leaching behavior of SX-113 sludge

\begin{tabular}{ccccccccccc}
\hline & $\begin{array}{c}\text { Liquid: } \\
\text { solids }\end{array}$ & NaOH & $\mathrm{T}$ & $\mathrm{T}$ Time & \multicolumn{7}{c}{ \% Removal } \\
\cline { 6 - 10 } Test & $(\mathrm{mL} / \mathrm{g})$ & $(M)$ & $\left({ }^{\circ} \mathrm{C}\right)$ & $(\mathrm{h})$ & $\mathrm{Al}$ & $\mathrm{Cr}$ & $\mathrm{P}$ & $\mathrm{U}$ & $\mathrm{Cs}$ \\
\hline 6 & 20 & 6.33 & 70 & 21 & 51.0 & 52.9 & 12.6 & 100. & 59.7 \\
10 & 44 & 6.33 & 80 & 126 & 78.7 & 65.5 & 91.7 & 93.1 & 86.2 \\
\hline
\end{tabular}

Table 4.23. Concentration of selected constituents in dry, untreated SX-113 sludge determined by different researchers

\begin{tabular}{cccc}
\hline Species & Unit of measure & LANL $^{\mathrm{a}}$ & ORNL $^{\mathrm{b}}$ \\
\hline $\mathrm{Al}$ & $\mu \mathrm{g} / \mathrm{g}$ & 21200. & 14000. \\
$\mathrm{Cr}$ & $\mu \mathrm{g} / \mathrm{g}$ & 113. & 110. \\
$\mathrm{P}$ & $\mu \mathrm{g} / \mathrm{g}$ & $<68.4$ & 7470. \\
$\mathrm{U}$ & $\mu \mathrm{g} / \mathrm{g}$ & 253. & 182. \\
$\mathrm{Cs}-137$ & $\mu \mathrm{Ci} / \mathrm{g}$ & 45.5 & 43.6 \\
$\mathrm{Pu}-239 / 240$ & $\mu \mathrm{Ci} / \mathrm{g}$ & 0.108 & 0.12 \\
\hline
\end{tabular}

${ }^{2}$ Temer and Villarreal, LAUR 97-2889 (1997).

'This work.

Table 4.24. Comparison of percentage of selected species removed from SX-113 sludge by different researchers

\begin{tabular}{cccc}
\hline & \multicolumn{3}{c}{ \% Removed $^{-}$} \\
\cline { 2 - 4 } Species & LANL $^{\mathrm{a}}$ & ORNL $^{\mathrm{b}}$ & ORNL $^{\mathrm{c}}$ \\
\hline $\mathrm{Al}$ & 89 & 51 & 79 \\
$\mathrm{Cr}$ & 40 & 53 & 66 \\
$\mathrm{P}$ & 85 & 13 & 92 \\
$\mathrm{U}$ & 88 & 100 & 93 \\
$\mathrm{Cs}$ & 88 & 60 & 86 \\
$\mathrm{Pu}$ & 0 & 39 & 45 \\
\hline
\end{tabular}

${ }^{\mathrm{a}}$ Temer and Villarreal, LAUR 97-2889 (1997); first leach $2.3 \mathrm{M} \mathrm{NaOH}, 5 \mathrm{~h}$, $100^{\circ} \mathrm{C}, 5 \mathrm{wt} \%$ solids; second leach $3.0 \mathrm{M} \mathrm{NaOH}, 5 \mathrm{~h}, 100^{\circ} \mathrm{C}, 1 \mathrm{wt} \%$ solids.

${ }^{\mathrm{b}}$ This work; $6.33 \mathrm{M} \mathrm{NaOH}, 21 \mathrm{~h}, 70^{\circ} \mathrm{C}$, and liquid:solids of $20: 1$.

'This work; $6.33 \mathrm{M} \mathrm{NaOH}, 126 \mathrm{~h}, 80^{\circ} \mathrm{C}$, and liquid:solids of $44: 1$. 
Table 4.25. Test \#7 - concentrations of species in enhanced sludge washing process steps for C-105 sludge (leach conditions: liquid:solids, $10 \mathrm{~mL} / \mathrm{g}$; $\mathrm{NaOH}, 6.33 \mathrm{M}$; temperature, $70^{\circ} \mathrm{C}$; time, $22 \mathrm{~h}$ )

\begin{tabular}{|c|c|c|c|c|c|c|c|c|}
\hline \multirow[b]{2}{*}{ Species } & \multicolumn{2}{|c|}{$\begin{array}{c}\text { Original sludge } \\
(\mu \mathrm{g} / \mathrm{g})\end{array}$} & \multicolumn{2}{|c|}{$\begin{array}{c}\text { Residual } \\
(\mu \mathrm{g} / \mathrm{g})\end{array}$} & \multicolumn{2}{|c|}{$\begin{array}{l}\text { Leachate } \\
(\mu \mathrm{g} / \mathrm{g})\end{array}$} & \multicolumn{2}{|c|}{$\begin{array}{l}\text { Rinse } \\
(\mu \mathrm{g} / \mathrm{g}) \\
\end{array}$} \\
\hline & Value & Std deviation & Value & Std deviation & Value & Std deviation & Value & Std deviation \\
\hline $\mathrm{Ag}$ & 8.87 & 0.199 & $<20.8^{a}$ & b & $<0.0902$ & b & $<0.102$ & b \\
\hline $\mathrm{Al}$ & 247000 & 503 & 18700 & 116 & 18100 & 75.6 & 160 & 0.672 \\
\hline $\mathrm{Ba}$ & 27.4 & 0.199 & 75.7 & 0.377 & 0.0496 & 0.0631 & $<0.0102$ & $b$ \\
\hline $\mathrm{Be}$ & $<0.0997$ & b & $<0.0943$ & $\mathrm{~b}$ & $<0.00451$ & $b$ & $<0.00509$ & $b$ \\
\hline $\mathrm{Bi}$ & 381 & 8.57 & 1180 & 11.1 & 0.334 & 2.08 & 5.26 & 4.03 \\
\hline $\mathrm{Ca}$ & 919 & 6.78 & 1130 & 5.28 & 1.25 & 0.153 & 7.61 & 0.336 \\
\hline $\mathrm{Cd}$ & 3.49 & 1.2 & 4.62 & 1.89 & 0.126 & 0.0722 & $<0.102$ & b \\
\hline Co & 4.09 & 0.797 & 13.3 & 0.189 & $<0.0857$ & $\mathrm{~b}$ & $<0.0967$ & b \\
\hline $\mathrm{CI}_{\mathrm{I}}$ & 405 & 3.19 & 332 & 0.755 & 28.2 & 0 & 0.229 & 0.0102 \\
\hline $\mathrm{Cu}$ & 703 & 4.78 & 1590 & 16.6 & 17.8 & 0.226 & $<0.0204$ & b \\
\hline $\mathrm{Fe}$ & 4020 & 20.1 & 9990 & 31.1 & 19.4 & 5.85 & 0.336 & 0 \\
\hline $\mathrm{K}$ & 447 & 1.4 & 31.3 & 1.89 & $<45.6$ & $\mathrm{~b}$ & 2.56 & 0.143 \\
\hline $\mathrm{Mg}$ & 31.2 & 2.59 & 41.5 & 4.15 & $<11.4$ & $b$ & $<0.127$ & b \\
\hline $\mathrm{Mn}$ & 1480 & 0 & 3800 & 10.4 & 0.293 & 0.334 & $<0.00509$ & $b$ \\
\hline $\mathrm{Ni}$ & 1450 & 161 & 4440 & 35.3 & 0.505 & 0.658 & $<0.102$ & $b$ \\
\hline $\mathrm{P}$ & 3590 & 4070 & 640 & 334 & 320 & 66.5 & 1.3 & 0.906 \\
\hline $\mathrm{Sb}$ & $<19.9$ & $\mathbf{b}$ & $<18.9$ & $b$ & $<91.1$ & $b$ & $<1.02$ & $\mathrm{~b}$ \\
\hline $\mathrm{Si}$ & 15000 & 0 & 22000 & 0 & c & $\mathrm{c}$ & c & c \\
\hline $\mathrm{Sr}$ & 95.6 & 0.399 & 318 & 2.08 & 0.0361 & 0.0271 & $<0.0255$ & $\mathrm{~b}$ \\
\hline Th & 319 & 3.39 & 992 & 8.68 & 0.537 & 0.559 & $<0.255$ & $b$ \\
\hline $\mathrm{U}$ & 12400 & 1030 & 38500 & 232 & 7.22 & 2.45 & $<0.509$ & b \\
\hline V & 8.77 & 0.199 & 9.06 & 0.377 & 0.41 & 0.00902 & $<0.0509$ & $\mathrm{~b}$ \\
\hline $\mathrm{Zn}$ & 206 & 2.59 & 108 & 1.51 & 5.78 & 0.0992 & $<0.153$ & $\mathrm{~b}$ \\
\hline Radiospecies & \multicolumn{2}{|c|}{$\mathrm{Bg} / \mathrm{g}$} & \multicolumn{2}{|c|}{$\mathrm{Ba} / \mathrm{g}$} & \multicolumn{2}{|c|}{$\mathrm{Bg} / \mathrm{g}$} & \multicolumn{2}{|c|}{$\mathrm{Bq} / \mathrm{g}$} \\
\hline Co-60 & 4600 & 1000 & 15000 & 2000 & 24.6 & c & 25.7 & $c$ \\
\hline Cs-137 & 11000000 & 1000000 & 7600000 & 100000 & 684000 & c & 8000 & c \\
\hline Eu-152 & $<3700$ & b & $<3800$ & $\mathrm{~b}$ & c & c & c & c \\
\hline Eu-154 & $<5500$ & b & 17000 & 0 & c & c & c & c \\
\hline Eu-155 & $<15000$ & $\mathrm{~b}$ & 33000 & 0 & c & c & c & c \\
\hline Pu-238 & 1000 & 100 & 1400 & 100 & c & $\mathrm{c}$ & $\mathrm{c}$ & c \\
\hline $\mathrm{Pu}-239 / 240$ & 43000 & 2000 & 110000 & 10000 & c & c & c & $\mathrm{c}$ \\
\hline Pu-all & 44000 & 2000 & 110000 & 10000 & c & c & c & c \\
\hline Am-241 & $<30000$ & b & 48000 & 0 & c & c & c & $\mathrm{c}$ \\
\hline Gross- $\alpha$ & 49000 & 16000 & 160000 & 30000 & 14 & 14 & 1.4 & 1.4 \\
\hline Gross- $\beta$ & 32000000 & 1000000 & 63000000 & 1000000 & 840000 & 10000 & 9800 & 100 \\
\hline
\end{tabular}
value.

The symbol < indicates that the concentration was below the detectable limit given by the following numerical

No standard deviation for values below the detectable limit.

Not measured. 
Table 4.26. Test \#7 - percentage of each species solubilized and percentage recovery for $\mathbf{C}-105$ sludge (leach conditions: liquid:solids, $10 \mathrm{~mL} / \mathrm{g}$; $\mathrm{NaOH}, 6.33 \mathrm{M}$; temperature, $70^{\circ} \mathrm{C}$; time, $22 \mathrm{~h}$ )

\begin{tabular}{|c|c|c|c|c|c|c|}
\hline \multirow[b]{2}{*}{ Species } & \multicolumn{2}{|c|}{$\begin{array}{c}\text { Based on residue } \\
(\%)\end{array}$} & \multicolumn{2}{|c|}{$\begin{array}{c}\text { Based on leachate \& rinse } \\
(\%)\end{array}$} & \multicolumn{2}{|c|}{$\begin{array}{c}\text { Recovery } \\
(\%) \\
\end{array}$} \\
\hline & Value & Std deviation & Value & Std deviation & Value & Std deviation \\
\hline $\mathrm{Ag}$ & 100.00 & 0.00 & 0.00 & 0.00 & 0.00 & 0.00 \\
\hline $\mathrm{Al}$ & 97.34 & 0.02 & 93.16 & 0.43 & 95.82 & 0.43 \\
\hline $\mathrm{Ba}$ & 3.03 & 0.85 & 2.25 & 2.87 & 99.22 & 2.99 \\
\hline $\mathrm{Be}$ & $\mathrm{a}$ & $\mathbf{a}$ & $a$ & $\mathrm{a}$ & $\mathrm{a}$ & $\mathrm{a}$ \\
\hline $\mathrm{Bi}$ & -8.71 & 2.65 & 43.14 & 32.94 & 151.85 & 33.12 \\
\hline $\mathrm{Ca}$ & 56.84 & 0.38 & 26.91 & 1.15 & 70.07 & 1.26 \\
\hline $\mathrm{Cd}$ & 53.54 & 24.83 & 44.93 & 30.02 & 91.39 & 44.85 \\
\hline Co & -14.14 & 22.30 & 0.00 & 0.00 & 114.14 & 22.30 \\
\hline $\mathrm{Cr}$ & 71.23 & 0.24 & 88.37 & 0.70 & 117.14 & 0.93 \\
\hline $\mathrm{Cu}$ & 20.61 & 0.99 & 31.51 & 0.45 & 110.89 & 1.19 \\
\hline $\mathrm{Fe}$ & 12.78 & 0.51 & 6.26 & 1.81 & 93.48 & 1.89 \\
\hline $\mathrm{K}$ & 97.54 & 0.15 & 17.44 & 0.98 & 19.90 & 0.99 \\
\hline $\mathrm{Mg}$ & 53.31 & 6.07 & 0.00 & 0.00 & 46.69 & 6.07 \\
\hline $\mathrm{Mn}$ & 9.88 & 0.25 & 0.25 & 0.28 & 90.37 & 0.37 \\
\hline $\mathrm{Ni}$ & -7.48 & 11.96 & 0.43 & 0.57 & 107.91 & 12.03 \\
\hline$P$ & 93.74 & 7.81 & 112.02 & 129.08 & 118.28 & 136.10 \\
\hline $\mathrm{Sb}$ & $\mathrm{a}$ & $\mathrm{a}$ & $\mathrm{a}$ & $\mathbf{a}$ & a & $\mathrm{a}$ \\
\hline $\mathrm{Si}$ & 48.52 & 0.00 & $0.00^{\mathrm{b}}$ & $0.00^{\mathrm{b}}$ & 51.48 & 0.00 \\
\hline $\mathrm{Sr}$ & -16.75 & 0.91 & 0.47 & 0.35 & 117.22 & 0.97 \\
\hline Th & -9.15 & 1.50 & 2.09 & 2.18 & 111.24 & 2.66 \\
\hline $\mathrm{U}$ & -8.98 & 9.08 & 0.72 & 0.25 & 109.70 & 9.14 \\
\hline V & 63.74 & 1.72 & 58.17 & 1.84 & 94.43 & 2.92 \\
\hline $\mathrm{Zn}$ & 81.60 & 0.35 & 34.91 & 0.74 & 53.32 & 0.94 \\
\hline \multicolumn{7}{|l|}{ Radiospecies } \\
\hline $\mathrm{Co}-60$ & -14.45 & 29.19 & 23.67 & 5.15 & 138.13 & 33.68 \\
\hline Cs-137 & 75.75 & 2.23 & 79.59 & 7.24 & 103.84 & 9.45 \\
\hline Eu-152 & $\mathbf{a}$ & $\mathrm{a}$ & $\mathbf{a}$ & $\mathbf{a}$ & a & a \\
\hline Eu-154 & a & a & a & a & a & a \\
\hline Eu-155 & $\mathbf{a}$ & $\mathbf{a}$ & a & a & $\mathbf{a}$ & a \\
\hline Pu-238 & 50.86 & 6.04 & $\mathrm{~b}$ & $b$ & 49.14 & 6.04 \\
\hline $\mathrm{Pu}-239 / 240$ & 10.21 & 9.17 & $\mathrm{~b}$ & $\mathrm{~b}$ & 89.79 & 9.17 \\
\hline Pu-all & 12.25 & 8.92 & $b$ & $b$ & 87.75 & 8.92 \\
\hline Am-241 & $\mathbf{a}$ & $\mathbf{a}$ & $\mathbf{a}$ & a & a & $\mathrm{a}$ \\
\hline Gross- $\alpha$ & -14.61 & 43.15 & 0.44 & 0.39 & 115.05 & 43.28 \\
\hline Gross- $\beta$ & 30.90 & 2.42 & 33.60 & 1.46 & 102.70 & 3.41 \\
\hline
\end{tabular}

Insufficient data for calculation; measured concentration in original sludge was below the detectable limit or was not measured.

beasurement was not made. 
chromium, $94 \%$ of the phosphorus, and $76 \%$ of the cesium were removed from the sludge. None of the uranium was removed. Values of recovery for these species indicate that the data are reliable.

Temer and Villarreal (1997) also analyzed and performed a caustic leaching test on this sludge. Their analysis of selected components of the sludge are compared with those of the present study in Table 4.27. There is good agreement between the two analyses for aluminum, chromium, cesium, and plutonium. Phosphorus and uranium showed significant differences. In their study, Temer and Villarreal (1997) leached sludge $\mathrm{C}-105$ with $\sim 3 \mathrm{MNaOH}$ at $100^{\circ} \mathrm{C}$. In this work, $\sim 6 \mathrm{M}$ $\mathrm{NaOH}$ at $70{ }^{\circ} \mathrm{C}$ was used, and the liquid:solids ratio was lower. Although the conditions varied considerably, and the initial analyses were different in some cases, the percentages of aluminum, phosphorus, and uranium leached from the sludge were about the same, as shown in Table 4.28. The percentages of chromium and cesium leached from the sludge were slightly lower at the conditions of the present study. The gross-alpha measurements did not confirm that plutonium was leached from the sludge.

\subsection{SLUDGE C-107}

One enhanced sludge washing test was performed with a sample of C-107 sludge. Analysis of this sludge (Table 4.29) indicates that the primary constituents are aluminum, bismuth, iron, phosphorus, and silicon. Uranium is $\sim 0.1 \mathrm{wt} \%$ of the wet sludge.

The measured concentrations of sludge components in the process streams are given in Table 4.29. Calculated values of the percentages of each species removed from the sludge and the percentage recovery are given in Table 4.30 . Approximately $82 \%$ of the aluminum, $70 \%$ of the chromium, $94 \%$ of the phosphorus, and $73 \%$ of the cesium were removed from the sludge by leaching with $6.33 \mathrm{MNaOH}$ at $70^{\circ} \mathrm{C}$ for $22 \mathrm{~h}$. The extent to which uranium and plutonium were leached was $1 \%$ and $6 \%$, respectively. The recovery of these six components was very good, providing a measure of confidence in the estimated fractions of each species removed from the sludge. Thorium was 
Table 4.27. Concentration of selected constituents in dry, untreated C-105 sludge determined by different researchers

\begin{tabular}{cccc}
\hline Species & Unit of measure & LANL $^{\mathrm{a}}$ & ORNL $^{\mathrm{b}}$ \\
\hline $\mathrm{Al}$ & $\mu \mathrm{g} / \mathrm{g}$ & 272000 & 276000. \\
$\mathrm{Cr}$ & $\mu \mathrm{g} / \mathrm{g}$ & 448. & 452. \\
$\mathrm{P}$ & $\mu \mathrm{g} / \mathrm{g}$ & 2000. & 4010. \\
$\mathrm{U}$ & $\mu \mathrm{g} / \mathrm{g}$ & 24900. & 13800. \\
$\mathrm{Cs}-137$ & $\mu \mathrm{Ci} / \mathrm{g}$ & 293. & 332. \\
$\mathrm{Pu}-239 / 240$ & $\mu \mathrm{Ci} / \mathrm{g}$ & 1.94 & 1.30 \\
\hline
\end{tabular}

${ }^{2}$ Temer and Villarreal, LAUR 97-2889 (1997).

bThis work.

Table 4.28. Comparison of percentage of selected species removed from C-105 sludge by different researchers

\begin{tabular}{ccc}
\hline & \multicolumn{2}{c}{ \% Removed } \\
\cline { 2 - 3 } Species & LANL $^{\mathrm{a}}$ & ORNL $^{\mathrm{b}}$ \\
\hline $\mathrm{Al}$ & 99 & 97 \\
$\mathrm{Cr}$ & 86 & 71 \\
$\mathrm{P}$ & 100 & 94 \\
$\mathrm{U}$ & 4 & 0 \\
$\mathrm{Cs}$ & 92 & 76 \\
$\mathrm{Pu}$ & 0 & 10 \\
\hline
\end{tabular}

${ }^{9}$ Temer and Villarreal, LAUR 97-2889 (1997); first leach $2.6 \mathrm{M} \mathrm{NaOH}, 5 \mathrm{~h}, 100^{\circ} \mathrm{C}, 5 \mathrm{wt} \%$ solids; second leach $3.1 \mathrm{M} \mathrm{NaOH}, 5 \mathrm{~h}, 100^{\circ} \mathrm{C}, 1.6 \mathrm{wt} \%$ solids.

'This work; $6.33 \mathrm{M} \mathrm{NaOH}, 22 \mathrm{~h}, 70^{\circ} \mathrm{C}$, and liquid:solids of 10:1. 
Table 4.29. Test \#8 - concentrations of species in enhanced sludge washing process steps for $\mathbf{C}$-107 sludge (leach conditions: liquid:solids, $6 \mathrm{~mL} / \mathrm{g}$; $\mathrm{NaOH}, 6.33 \mathrm{M}$; temperature, $70^{\circ} \mathrm{C}$; time, $22 \mathrm{~h}$ )

\begin{tabular}{|c|c|c|c|c|c|c|c|c|}
\hline \multirow[b]{2}{*}{ Species } & \multicolumn{2}{|c|}{$\begin{array}{c}\text { Original sludge } \\
(\mu \mathrm{g} / \mathrm{g})\end{array}$} & \multicolumn{2}{|c|}{$\begin{array}{l}\text { Residual } \\
(\mu \mathrm{g} / \mathrm{g})\end{array}$} & \multicolumn{2}{|c|}{$\begin{array}{l}\text { Leachate } \\
(\mu \mathrm{g} / \mathrm{g})\end{array}$} & \multicolumn{2}{|c|}{$\begin{array}{l}\text { Rinse } \\
(\mu \mathrm{g} / \mathrm{g})\end{array}$} \\
\hline & Value & Std deviation & Value & Std deviation & Value & Std deviation & Value & Std deviation \\
\hline $\mathrm{Ag}$ & 7.04 & 0.515 & 373 & 2.96 & $<0.0838^{\mathrm{a}}$ & $\mathrm{b}$ & 0.147 & 0 \\
\hline $\mathrm{Al}$ & 56600 & 303 & 20900 & 144 & 7060 & 16.9 & 319 & 10.9 \\
\hline $\mathrm{Ba}$ & 312 & 3.61 & 546 & 3.94 & 0.021 & 0.0168 & $<0.00978$ & $\mathrm{~b}$ \\
\hline $\mathrm{Be}$ & $<0.0859$ & $\mathrm{~b}$ & $<0.0235$ & b & $<0.00419$ & $\mathrm{~b}$ & $<0.00489$ & $\mathrm{~b}$ \\
\hline $\mathrm{Bi}$ & 10500 & 245 & 20200 & 208 & 163 & 7.62 & 70.1 & 43.5 \\
\hline $\mathrm{Ca}$ & 1090 & 21.6 & 1270 & 13.8 & 0.482 & 0.0335 & 65.7 & 3.95 \\
\hline $\mathrm{Cd}$ & 43 & 1.03 & 75.5 & 0.704 & $<0.0838$ & $b$ & $<0.0978$ & $b$ \\
\hline Co & 9.19 & 0 & 15.4 & 0.141 & $<0.0796$ & $b$ & $<0.0929$ & b \\
\hline $\mathrm{Cr}_{\mathrm{r}}$ & 518 & 10.8 & 315 & 3.94 & 53.3 & 0.846 & 2.43 & 0.0293 \\
\hline $\mathrm{Cu}$ & 90.4 & 0.859 & 98.2 & 0.704 & 3.79 & 0.0168 & 0.103 & 0.00978 \\
\hline $\mathrm{Fe}$ & 37100 & 256 & 57400 & 232 & 13.1 & 0.846 & 0.171 & 0.00978 \\
\hline $\mathrm{K}$ & 300 & 7.56 & 20.1 & 1.08 & $<42.3$ & $\mathrm{~b}$ & 4.8 & 0.372 \\
\hline $\mathrm{Mg}$ & 290 & 10.8 & 503 & 10.8 & $<0.105$ & $b$ & $<0.122$ & b \\
\hline Mn & 1870 & 10.8 & 2990 & 17.7 & 0.0461 & 0.0754 & $<0.00489$ & b \\
\hline $\mathrm{Ni}$ & 3260 & 61.3 & 6140 & 65.1 & $<0.0838$ & $b$ & $<0.0978$ & $b$ \\
\hline $\mathrm{P}$ & 14800 & 343 & 1870 & 437 & 474 & 67.7 & 1270 & 118 \\
\hline $\mathrm{Sb}$ & 596 & 5.84 & $<98.6$ & $\mathrm{~b}$ & $<0.838$ & $b$ & $<0.978$ & $\mathrm{~b}$ \\
\hline $\mathrm{Si}$ & 29000 & 0 & 75000 & 0 & c & $\mathrm{c}$ & c & c \\
\hline $\mathrm{Sr}$ & 189 & 3.61 & 360 & 0.986 & 0.0461 & 0.0335 & $<0.0245$ & $\mathrm{~b}$ \\
\hline Th & 532 & 7.9 & 554 & 36.5 & 1.21 & 0.251 & $<0.245$ & $\mathrm{~b}$ \\
\hline $\mathrm{U}$ & 9660 & 112 & 19700 & 171 & 42.5 & 0.411 & 1.64 & 0.215 \\
\hline $\mathrm{V}$ & 8.93 & 0.515 & 12.3 & 0.986 & $<0.0419$ & $\mathrm{~b}$ & 0.0831 & 0 \\
\hline $\mathrm{Zn}$ & 137 & 9.79 & 115 & 0.704 & 9.03 & 0.0754 & 0.308 & 0.0685 \\
\hline Radiospecies & \multicolumn{2}{|c|}{$\mathrm{Bg} / \mathrm{g}$} & \multicolumn{2}{|c|}{$\mathrm{Bg} / \mathrm{g}$} & \multicolumn{2}{|c|}{$\mathrm{Bq} / \mathrm{g}$} & \multicolumn{2}{|c|}{$\mathrm{Bg} / \mathrm{g}$} \\
\hline Co-60 & 12000 & 1000 & 20000 & 4000 & 25.4 & c & 22.1 & c \\
\hline Cs-137 & 1600000 & 100000 & 900000 & 20000 & 175000 & c & 9180 & c \\
\hline Eu-152 & $<4000$ & b & $<8800$ & $\mathrm{~b}$ & c & c & c & $c$ \\
\hline Eu-154 & 94000 & 7000 & 170000 & 20000 & c & c & c & c \\
\hline Eu-155 & 79000 & 14000 & 170000 & 30000 & c & c & c & c \\
\hline Pu-238 & 11000 & 1000 & 22000 & 2000 & c & c & c & c \\
\hline $\mathrm{Pu}-239 / 240$ & 51000 & 2000 & 98000 & 8000 & c & c & c & c \\
\hline Pu-all & 62000 & 2000 & 120000 & 10000 & c & c & c & c \\
\hline Am-241 & 84000 & 22000 & 180000 & 70000 & $c$ & c & c & $\mathrm{c}$ \\
\hline Gross- $\alpha$ & 190000 & 30000 & 290000 & 60000 & 240 & 50 & 6 & 11 \\
\hline Gross- $\beta$ & 130000000 & 10000000 & 250000000 & 10000000 & 230000 & 10000 & 14000 & 1000 \\
\hline
\end{tabular}

"The symbol < indicates that the concentration was below the detectable limit given by the following numerical value.

No standard deviation for values below the detectable limit.

Not measured. 
Table 4.30. Test \#8 - percentage of each species solubilized and percentage recovery for C-107 sludge (leach conditions: liquid:solids, $6 \mathrm{~mL} / \mathrm{g} ; \mathrm{NaOH}, 6.33 \mathrm{M}$; temperature, $70^{\circ} \mathrm{C}$; time, $22 \mathrm{~h}$ )

\begin{tabular}{|c|c|c|c|c|c|c|}
\hline \multirow[b]{2}{*}{ Species } & \multicolumn{2}{|c|}{$\begin{array}{c}\text { Based on residue } \\
(\%) \\
\end{array}$} & \multicolumn{2}{|c|}{$\begin{array}{c}\text { Based on leachate \& rinse } \\
(\%)\end{array}$} & \multicolumn{2}{|c|}{$\begin{array}{c}\text { Recovery } \\
(\%)\end{array}$} \\
\hline & Value & Std deviation & Value & Std deviation & Value & Std deviation \\
\hline $\mathrm{Ag}$ & -2482.46 & 190.02 & 21.22 & 1.55 & 2603.69 & 191.57 \\
\hline $\mathrm{Al}$ & 82.00 & 0.16 & 79.71 & 0.50 & 97.71 & 0.60 \\
\hline $\mathrm{Ba}$ & 14.70 & 1.16 & 0.04 & 0.03 & 85.34 & 1.16 \\
\hline $\mathrm{Be}$ & a & $\mathrm{a}$ & a & a & $a$ & a \\
\hline $\mathrm{Bi}$ & 6.23 & 2.39 & 15.99 & 4.25 & 109.76 & 5.04 \\
\hline $\mathrm{Ca}$ & 43.21 & 1.28 & 61.53 & 3.88 & 118.32 & 4.41 \\
\hline $\mathrm{Cd}$ & 14.42 & 2.20 & 0.00 & 0.00 & 85.58 & 2.20 \\
\hline $\mathrm{Co}$ & 18.32 & 0.75 & 0.00 & 0.00 & 81.68 & 0.75 \\
\hline $\mathrm{Cr}$ & 70.36 & 0.72 & 65.80 & 1.68 & 95.44 & 2.24 \\
\hline $\mathrm{Cu}$ & 47.05 & 0.63 & 26.03 & 0.29 & 78.97 & 0.86 \\
\hline $\mathrm{Fe}$ & 24.59 & 0.60 & 0.21 & 0.01 & 75.63 & 0.60 \\
\hline $\mathrm{K}$ & 96.73 & 0.19 & 16.26 & 1.33 & 19.53 & 1.36 \\
\hline $\mathrm{Mg}$ & 15.46 & 3.63 & 0.00 & 0.00 & 84.54 & 3.63 \\
\hline $\mathrm{Mn}$ & 22.07 & 0.64 & 0.01 & 0.02 & 77.95 & 0.65 \\
\hline $\mathrm{Ni}$ & 8.20 & 1.98 & $0.00^{b}$ & $0.00^{b}$ & 91.80 & 1.98 \\
\hline $\mathrm{P}$ & 93.84 & 1.45 & 106.22 & 8.89 & 112.38 & 9.05 \\
\hline $\mathrm{Sb}$ & 100.00 & 0.00 & 0.00 & 0.00 & 0.00 & 0.00 \\
\hline $\mathrm{Si}$ & -26.06 & 0.00 & 0.00 & 0.00 & 126.06 & 0.00 \\
\hline Sr & 7.16 & 1.79 & 0.14 & 0.11 & 92.99 & 1.80 \\
\hline Th & 49.24 & 3.43 & 1.35 & 0.28 & 52.11 & 3.44 \\
\hline $\mathrm{U}$ & 0.60 & 1.44 & 2.78 & 0.05 & 102.18 & 1.47 \\
\hline V & 32.86 & 6.63 & 9.46 & 0.55 & 76.59 & 6.96 \\
\hline $\mathrm{Zn}$ & 59.09 & 2.93 & 41.38 & 3.02 & 82.29 & 5.92 \\
\hline \multicolumn{7}{|c|}{ Radiospecies } \\
\hline Co-60 & .18 .76 & 17.60 & 3.13 & 0.26 & 84.36 & 17.70 \\
\hline Cs-137 & 72.58 & 1.82 & 70.71 & 4.42 & 98.12 & 6.16 \\
\hline Eu-152 & a & a & a & a & $\mathbf{a}$ & a \\
\hline Eu-154 & 11.85 & 12.27 & b & b & 88.15 & 12.27 \\
\hline Eu-155 & -4.89 & 26.23 & b & b & 104.89 & 26.23 \\
\hline Pu-238 & 2.52 & 12.53 & $\mathrm{~b}$ & $\mathrm{~b}$ & 97.48 & 12.53 \\
\hline $\mathrm{Pu}-239 / 240$ & 6.34 & 8.48 & $\mathrm{~b}$ & $\mathrm{~b}$ & 93.66 & 8.48 \\
\hline Pu-all & 5.66 & 8.43 & $\mathrm{~b}$ & $b$ & 94.34 & 8.43 \\
\hline $\mathrm{Am}-241$ & -4.45 & 48.97 & $\mathrm{~b}$ & $\mathrm{~b}$ & 104.45 & 48.97 \\
\hline Gross-a & 25.61 & 19.36 & 0.78 & 0.21 & 75.18 & · 19.44 \\
\hline Gross-b & 6.27 & 8.13 & 1.16 & 0.10 & 94.89 & 8.21 \\
\hline
\end{tabular}

'Insufficient data for calculation; measured concentration in original sludge was below the detectable limit or was not measured.

'Measurement was not made. 
probably not removed to $49 \%$ because the relatively low value of its recovery, $52 \%$, could indicate that more thorium remained in the residue than was measured.

Concentrations of selected constituents in C-107 sludge as measured in this work and by other researchers [Temer and Villarreal (1996) and Lumetta et al. (1996)] are compared in Table 4.31. The wide variation in concentrations of the components determined at different laboratories may indicate that the samples were different as a consequence of the sludge within the tank being inhomogeneous. A comparison of the percentages of each constituent removed from the sludge by the different researchers is shown in Table 4.32. These numbers are generally consistent, except for uranium. The percentage removed is, by definition, scaled to the concentrations in the original sludge, so the variations in percentage removed can be attributed to the different leaching conditions employed. With leaching conditions ranging from $3 \mathrm{M}$ to $6.33 \mathrm{M} \mathrm{NaOH}, 70$ to $100^{\circ} \mathrm{C}$, and 10 to $22 \mathrm{~h}, 63$ to $82 \%$ of the aluminum, 48 to $70 \%$ of the chromium, 91 to $94 \%$ of the phosphorus, and 70 to $73 \%$ of the cesium were removed from the sludge.

\subsection{SLUDGE C-104}

C-104 sludge samples arrived at ORNL in two bottles. Sludge in one bottle contained a small piece of hard material resembling concrete; the remainder of the sludge was the typical slurry-like material and had a water content of $63.5 \mathrm{wt} \%$. This sludge (without the "rock") was used in tests \#11 and \#13. Sludge in the other bottle was of the usual consistency and had a water content of $43.7 \mathrm{wt} \%$ and was used in test \#12. Samples from each bottle were analyzed to determine whether the sludge was different. The analyses of the wet samples are given in Tables 4.33 and 4.35 as part of the enhanced sludge washing partitioning data. There are small differences in the concentrations of most species in the two samples (even on a dry basis, as shown later in Table 4.40). The major constituents of sludge C-104 are aluminum, iron, silicon, thorium, and uranium. Bismuth and phosphorus concentrations are low. 
Table 4.31. Concentration of selected constituents in dry, untreated C-107 sludge determined by different researchers

\begin{tabular}{cccccc}
\hline Species & Unit of measure & LANL $^{\mathrm{a}}$ & PNNL $^{\mathrm{b}}$ & PNNL $^{\mathrm{c}}$ & ORNL $^{\mathrm{d}}$ \\
\hline $\mathrm{Al}$ & $\mu \mathrm{g} / \mathrm{g}$ & 105000 & 86800 & 34400. & 60500. \\
$\mathrm{Cr}$ & $\mu \mathrm{g} / \mathrm{g}$ & 706. & 1250. & 595. & 553. \\
$\mathrm{P}$ & $\mu \mathrm{g} / \mathrm{g}$ & 36500. & 9500. & 11800. & 15800. \\
$\mathrm{U}$ & $\mu \mathrm{g} / \mathrm{g}$ & 23500. & 11100. & 6450. & 10300. \\
$\mathrm{Cs}-137$ & $\mu \mathrm{Ci} / \mathrm{g}$ & 75.4 & 127. & 57.4 & 46.2 \\
$\mathrm{Pu}-239 / 240$ & $\mu \mathrm{Ci} / \mathrm{g}$ & 2.90 & 5.77 & & 1.47 \\
\hline
\end{tabular}

${ }^{2}$ Temer and Villarreal, LAUR 96-2839 (1996).

bLumetta et al., PNNL-11278 (1996).

'Brooks et al., Letter Report (September 1996).

dThis work.

Table 4.32. Comparison of percentage of selected species removed from C-107 sludge by different researchers

\begin{tabular}{ccccc}
\hline & \multicolumn{3}{c}{ \% Removed } \\
\cline { 2 - 4 } Species & LANL $^{\mathrm{a}}$ & PNNL $^{\mathrm{b}}$ & PNNL $^{\mathrm{c}}$ & ORNL $^{\mathrm{d}}$ \\
$\mathrm{Al}$ & 76 & 78 & 63 & 82 \\
$\mathrm{Cr}$ & 68 & 48 & $64-67$ & 70 \\
$\mathrm{P}$ & 94 & 94 & 91 & 94 \\
$\mathrm{U}$ & 22 & 22 & $31-34$ & 1 \\
$\mathrm{Cs}$ & 73 & 70 & 71 & 73 \\
$\mathrm{Pu}$ & 0 & 2 & & 6 \\
\hline
\end{tabular}

${ }^{8}$ Temer and Villarreal, LAUR $96-2839(1996) ; 3.0 \mathrm{M}$ NaOH, $10 \mathrm{~h}, 100^{\circ} \mathrm{C}, 8 \mathrm{wt} \%$ solids.

'Lumetta et al., PNNL-11278 (1996); 3.0 M NaOH, $10 \mathrm{~h}, 100^{\circ} \mathrm{C}, 8 \mathrm{wt} \%$ solids.

'Brooks et al., Letter Report (September 1996); $1.2 \mathrm{MNaOH}, 5 \mathrm{~h}, 100^{\circ} \mathrm{C}, 5.4 \mathrm{wt} \%$ solids; second leach $3.5 \mathrm{M} \mathrm{NaOH}, 5 \mathrm{~h}, 100^{\circ} \mathrm{C}, 5.8 \mathrm{wt} \%$ solids

${ }^{d}$ This work; $6.33 \mathrm{M} \mathrm{NaOH}, 22 \mathrm{~h}, 70^{\circ} \mathrm{C}$, liquid:solids = 6:1. 
As indicated above, three enhanced sludge washing tests were performed with C-104 sludge. In each of these tests the sodium hydroxide concentration was $4 M$ and the leaching time was $\sim 64 \mathrm{~h}$. The primary variables were temperature and liquid:solids ratio, which varied from 50 to $93^{\circ} \mathrm{C}$ and from 19 to $31 \mathrm{~mL} / \mathrm{g}$, respectively. Concentrations measured in each process stream and calculated values of the percentage of each constituent removed from the sludge are given in Tables 4.33 through 4.38. The operating conditions and the percentages of selected constituents removed from the sludge in the three tests are summarized in Table 4.39. Data on the percentage of phosphorus removed from the sludge is useless. Difficulty in obtaining good values may have been caused by the initially low concentration of phosphorus in the sludge. The percentages of chromium, uranium, and cesium removed from the sludge increased in lock-step with operating temperature. The same trend was not seen with aluminum, presumably due to the poor recovery of aluminum in test $\# 12$ (80\% versus $>90 \%$ in the other two tests). Based on the analysis of the residue, about $23 \%$ of the thorium was removed from the sludge; but again the analysis of the leachate did not corroborate this.

The concentrations of selected constituents in C-104 sludge (on a dry basis) determined in this work are compared with measurements made by Temer and Villarreal (1997) in Table 4.40. The analysis of the sludge sample with low water content compares favorably with the results of Temer and Villarreal. With the exception of phosphorus, the high-water-content sample has higher concentrations of the metals in the selected subset. This seeming contradiction may be an artifact of too high a value for the water content which is used to adjust the concentrations to a dry basis.

The percentage of species removed from the sludge in these tests are compared to the findings of Temer and Villarreal (1997) in Table 4.41. Clearly, increasing the temperature from $50^{\circ} \mathrm{C}$ to above $90^{\circ} \mathrm{C}$ improves the removal of aluminum, chromium, and cesium. There is poor agreement on the removal of uranium. 
Table 4.33. Test \#11 - concentrations of species in enhanced sludge washing process steps for C-104 sludge (leach conditions: liquid: solids, $31 \mathrm{~mL} / \mathrm{g}$; $\mathrm{NaOH}, 4 \mathrm{M}$; temperature, $80^{\circ} \mathrm{C}$; time, $65 \mathrm{~h}$ )

\begin{tabular}{|c|c|c|c|c|c|c|c|c|}
\hline \multirow[b]{2}{*}{ Species } & \multicolumn{2}{|c|}{$\begin{array}{c}\text { Original sludge } \\
(\mu \mathrm{g} / \mathrm{g})\end{array}$} & \multicolumn{2}{|c|}{$\begin{array}{l}\text { Residual } \\
(\mu \mathrm{g} / \mathrm{g})\end{array}$} & \multicolumn{2}{|c|}{$\begin{array}{c}\text { Leachate } \\
(\mu \mathrm{g} / \mathrm{g})\end{array}$} & \multicolumn{2}{|c|}{$\begin{array}{l}\text { Rinse } \\
(\mu \mathrm{g} / \mathrm{g})\end{array}$} \\
\hline & Value & Std deviation & Value & Std deviation & Value & Std deviation & Value & Std deviation \\
\hline $\mathrm{Ag}$ & $<1.53^{\mathrm{a}}$ & $\mathrm{b}$ & $<5.05$ & $\mathrm{~b}$ & 0.323 & 0.091 & $<0.103$ & $\mathrm{~b}$ \\
\hline $\mathrm{Al}$ & 31700 & 3170 & 22800 & 2280 & 441 & 44.1 & 17.3 & 1.73 \\
\hline $\mathrm{Ba}$ & 120 & 12 & 110 & 11 & 0.0319 & 0.0364 & $<0.0103$ & $\mathrm{~b}$ \\
\hline $\mathrm{Be}$ & 34.1 & 3.41 & 10.3 & 1.03 & 1.25 & 0.125 & $<0.00517$ & $\mathrm{~b}$ \\
\hline $\mathrm{Bi}$ & 27.7 & 11.8 & 23.7 & 4.97 & $<0.0228$ & $\mathrm{~b}$ & $<0.0259$ & $\mathrm{~b}$ \\
\hline $\mathrm{Ca}$ & 1940 & 194 & 1510 & 151 & 0.0501 & 0.437 & 0.429 & 0.0429 \\
\hline $\mathrm{Cd}$ & 1030 & 103 & 881 & 88.1 & 1.41 & 0.246 & $<0.103$ & $\mathrm{~b}$ \\
\hline Co & 10.2 & 1.02 & 7.32 & 2.52 & $<0.0865$ & $b$ & $<0.0982$ & $\mathrm{~b}$ \\
\hline $\mathrm{Cr}$ & 1130 & 113 & 462 & 46.2 & 31.7 & 3.17 & 1.21 & 0.121 \\
\hline $\mathrm{Cu}$ & 31.9 & 3.36 & 21.4 & 2.14 & 0.168 & 0.0168 & 0.119 & 0.0207 \\
\hline $\mathrm{Fe}$ & 21700 & 2170 & 17400 & 1740 & 7.56 & 4.71 & 0.719 & 0.3 \\
\hline $\mathrm{K}$ & 643 & 64.3 & 107 & 10.7 & 96.6 & 9.66 & 12.8 & 1.28 \\
\hline $\mathrm{Mg}$ & 387 & 48.7 & 303 & 30.3 & $<0.114$ & $\mathrm{~b}$ & $<0.129$ & $b$ \\
\hline $\mathrm{Mn}$ & 4140 & 414 & 3340 & 334 & 0.601 & 0.956 & 0.0259 & 0.062 \\
\hline $\mathrm{Ni}$ & 2090 & 209 & 1750 & 175 & 2.09 & 0.573 & $<0.103$ & $\mathrm{~b}$ \\
\hline $\mathrm{P}$ & 77 & 104 & 233 & 400 & 231 & 36 & 3.47 & 3.47 \\
\hline $\mathrm{Sb}$ & 4.22 & 4.58 & 0.853 & 0.165 & $<0.0228$ & b & $<0.0259$ & b \\
\hline Si & 8500 & 0 & 56000 & 0 & c & c & c & c \\
\hline $\mathrm{Sr}$ & 51.2 & 5.12 & 41.8 & 4.18 & $<0.0228$ & $\mathbf{b}$ & $<0.0259$ & $\mathbf{b}$ \\
\hline Th & 11600 & 1160 & 8950 & 895 & 2.17 & 2.57 & 0.346 & 0.455 \\
\hline$U$ & 20200 & 2020 & 16900 & 3640 & 15.7 & 4.81 & $<0.517$ & $\mathrm{~b}$ \\
\hline $\mathrm{V}$ & 4.58 & 0.458 & $<0.229$ & $\mathrm{~b}$ & 0.237 & 0.0237 & $<0.0517$ & $\mathbf{b}$ \\
\hline $\mathrm{Zn}$ & 62.6 & 6.26 & 40.1 & 4.01 & 0.805 & 0.0805 & 0.191 & 0.0517 \\
\hline Radiospecies & \multicolumn{2}{|c|}{$\mathrm{Bg} / \mathrm{g}$} & \multicolumn{2}{|c|}{$\mathrm{Bg} / \mathrm{g}$} & \multicolumn{2}{|c|}{$\mathrm{Bg} / \mathrm{g}$} & \multicolumn{2}{|c|}{$\mathrm{Bg} / \mathrm{g}$} \\
\hline Co-60 & 6100 & 3700 & 4200 & 1300 & $<310$ & b & $<35$ & $b$ \\
\hline Cs-137 & 2700000 & 100000 & 970000 & 20000 & 110000 & 10000 & 4200 & 200 \\
\hline Eu-152 & $<4700$ & $\mathbf{b}$ & $<6200$ & b & $<950$ & b & $<140$ & $b$ \\
\hline Eu-154 & 18000 & 8000 & 16000 & 5000 & $<410$ & $b$ & $<31$ & $b$ \\
\hline Eu-155 & 23000 & 25000 & 19000 & 11000 & $<970$ & $\mathrm{~b}$ & $<67$ & $b$ \\
\hline Pu-238 & 9300 & 100 & 6100 & 100 & c & c & $c$ & c \\
\hline $\mathrm{Pu}-239 / 240$ & 59000 & 1000 & 40000 & 1000 & c & c & c & c \\
\hline Pu-all & 68000 & 1000 & 46000 & 1000 & $c$ & c & $\mathrm{c}$ & c \\
\hline Am-241 & 86000 & 49000 & 110000 & 20000 & $<2000$ & $\mathrm{~b}$ & $<160$ & \\
\hline Gross- $\alpha$ & 210000 & 10000 & 170000 & 10000 & c & $c$ & $\mathbf{c}$ & $c$ \\
\hline Gross- $\beta$ & 30000000 & 1000000 & 21000000 & 1000000 & 120000 & 10000 & 4700 & 100 \\
\hline
\end{tabular}

The symbol < indicates that the concentration was below the detectable limit given by the following numerical value. No standard deviation for values below the detectable limit.

Not measured. 
Table 4.34. Test \#11 - percentage of each species solubilized and percentage recovery for $\mathrm{C}$-104 sludge (leach conditions: liquid:solids, $31 \mathrm{~mL} / \mathrm{g}$; $\mathrm{NaOH}, 4 \mathrm{M}$; temperature, $80^{\circ} \mathrm{C}$; time, $65 \mathrm{~h}$ )

\begin{tabular}{|c|c|c|c|c|c|c|}
\hline \multirow[b]{2}{*}{ Species } & \multicolumn{2}{|c|}{$\begin{array}{c}\text { Based on residue } \\
(\%) \\
\end{array}$} & \multicolumn{2}{|c|}{$\begin{array}{c}\text { Based on leachate \& rinse } \\
(\%)\end{array}$} & \multicolumn{2}{|c|}{$\begin{array}{c}\text { Recovery } \\
(\%) \\
\end{array}$} \\
\hline & Value & Std deviation & Value & Std deviation & Value & Std deviation \\
\hline $\mathrm{Ag}$ & $\mathbf{a}$ & $\mathbf{a}$ & $\mathrm{a}$ & $\mathrm{a}$ & $\mathrm{a}$ & $\mathrm{a}$ \\
\hline $\mathrm{Al}$ & 28.73 & 10.08 & 19.36 & 2.66 & 90.63 & 11.67 \\
\hline $\mathrm{Ba}$ & 9.17 & 12.85 & 0.35 & 0.40 & 91.18 & 12.88 \\
\hline $\mathrm{Be}$ & 70.07 & 4.23 & 47.85 & 6.77 & 77.78 & 9.61 \\
\hline $\mathrm{Bi}$ & 15.22 & 40.26 & 0.00 & 0.00 & 84.78 & 40.26 \\
\hline $\mathrm{Ca}$ & 22.87 & 10.91 & 0.52 & 0.30 & 77.65 & 10.95 \\
\hline $\mathrm{Cd}$ & 15.24 & 11.99 & 1.79 & 0.36 & 86.54 & 12.12 \\
\hline Co & 28.89 & 25.49 & 0.00 & 0.00 & 71.11 & 25.49 \\
\hline $\mathrm{Cr}$ & 59.49 & 5.73 & 38.98 & 5.35 & 79.49 & 9.65 \\
\hline $\mathrm{Cu}$ & 33.53 & 9.65 & 15.09 & 2.24 & 81.56 & 10.98 \\
\hline $\mathrm{Fe}$ & 20.54 & 11.24 & 0.53 & 0.29 & 79.98 & 11.28 \\
\hline $\mathrm{K}$ & 83.51 & 2.33 & 239.94 & 31.30 & 256.42 & 32.62 \\
\hline $\mathrm{Mg}$ & 22.42 & 12.47 & 0.00 & 0.00 & 77.58 & 12.47 \\
\hline Mn & 20.06 & 11.31 & 0.20 & 0.30 & 80.15 & 11.32 \\
\hline $\mathrm{Ni}$ & 17.03 & 11.73 & 1.31 & 0.38 & 84.28 & 11.83 \\
\hline $\mathrm{P}$ & -199.85 & 654.97 & 4015.55 & 5458.73 & 4315.39 & 5883.85 \\
\hline $\mathrm{Sb}$ & 79.97 & 22.08 & 0.00 & 0.00 & 20.03 & 22.08 \\
\hline $\mathrm{Si}$ & -552.83 & 0.00 & $\mathrm{~b}$ & b & 652.83 & 0.00 \\
\hline $\mathrm{Sr}$ & 19.10 & 11.44 & 0.00 & 0.00 & 80.90 & 11.44 \\
\hline Th & 23.55 & 10.81 & 0.31 & 0.30 & 76.76 & 10.84 \\
\hline $\mathrm{U}$ & 17.10 & 19.69 & 1.01 & 0.33 & 83.92 & 19.73 \\
\hline V & 100.00 & 0.00 & 67.55 & 9.55 & 67.55 & 9.55 \\
\hline $\mathrm{Zn}$ & 36.52 & 8.98 & 23.50 & 3.41 & 86.98 & 11.05 \\
\hline \multicolumn{7}{|l|}{ Radiospecies } \\
\hline Co-60 & 31.77 & 46.46 & 0.00 & 0.00 & 68.23 & 46.46 \\
\hline Cs-137 & 64.40 & 1.51 & 56.61 & 5.27 & 92.21 & 5.97 \\
\hline Eu-152 & $\mathrm{a}$ & a & $\mathrm{a}$ & a & a & $\mathbf{a}$ \\
\hline Eu-154 & 11.92 & 47.86 & 0.00 & 0.00 & 88.08 & 47.86 \\
\hline Eu-155 & 18.14 & 100.81 & 0.00 & 0.00 & 81.86 & 100.81 \\
\hline $\mathrm{Pu}-238$ & 35.00 & 1.27 & $\mathrm{~b}$ & $\mathrm{~b}$ & 65.00 & 1.27 \\
\hline $\mathrm{Pu}-239 / 240$ & 32.82 & 2.03 & $\mathrm{~b}$ & $\mathrm{~b}$ & 67.18 & 2.03 \\
\hline Pu-all & 32.97 & 1.76 & b. & $\mathrm{b}$ & 67.03 & 1.76 \\
\hline Am-241 & -26.74 & 75.80 & 0.00 & 0.00 & 126.74 & 75.80 \\
\hline Gross- $\alpha$ & 19.78 & 6.07 & $\mathrm{~b}$ & $\mathrm{~b}$ & 80.22 & 6.07 \\
\hline Gross- $\beta$ & 30.64 & 4.03 & 5.57 & 0.47 & 74.93 & 4.16 \\
\hline
\end{tabular}

Insufficient data for calculation; measured concentration in original sludge was below the detectable limit or was not measured.

'Measurement was not made. 
Table 4.35. Test \#12 - concentrations of species in enhanced sludge washing process steps for $\mathbf{C}-104$ sludge (leach conditions: liquid:solids, $19 \mathrm{~mL} / \mathrm{g} ; \mathrm{NaOH}, 4 \mathrm{M}$; temperature, $50^{\circ} \mathrm{C}$; time, $63 \mathrm{~h}$ )

\begin{tabular}{|c|c|c|c|c|c|c|c|c|}
\hline \multirow[b]{2}{*}{ Species } & \multicolumn{2}{|c|}{$\begin{array}{l}\text { Original sludge } \\
(\mu \mathrm{g} / \mathrm{g})\end{array}$} & \multicolumn{2}{|c|}{$\begin{array}{l}\text { Residual } \\
(\mu \mathrm{g} / \mathrm{g})\end{array}$} & \multicolumn{2}{|c|}{$\begin{array}{l}\text { Leachate } \\
(\mu \mathrm{g} / \mathrm{g})\end{array}$} & \multicolumn{2}{|c|}{$\begin{array}{l}\text { Rinse } \\
(\mu \mathrm{g} / \mathrm{g})\end{array}$} \\
\hline & Value & Std deviation & Value & Std deviation & Value & Std deviation & Value & Std deviation \\
\hline $\mathrm{Ag}$ & 60.7 & 6.07 & 183 & 18.3 & 1.34 & 0.134 & $<0.209^{2}$ & b \\
\hline $\mathrm{Al}$ & 36000 & 3600 & 19900 & 1990 & 559 & 55.9 & 16.5 & 1.65 \\
\hline $\mathrm{Ba}$ & 113 & 11.3 & 90.3 & 9.03 & $<0.0182$ & $\mathrm{~b}$ & $<0.0209$ & $\mathrm{~b}$ \\
\hline $\mathrm{Be}$ & 33 & 3.3 & 12.2 & 1.22 & 1.26 & 0.126 & $<0.0104$ & $b$ \\
\hline $\mathrm{Ca}$ & 2080 & 208 & 1410 & 141 & 1.23 & 0.123 & 1.65 & 0.165 \\
\hline $\mathrm{Cd}$ & 952 & 95.2 & 712 & 71.2 & 1.88 & 0.188 & 0.282 & 0.188 \\
\hline Co & 6.19 & 0.619 & 5.03 & 0.51 & $<0.173$ & b & $<0.198$ & $\mathrm{~b}$ \\
\hline $\mathrm{Cr}$ & 999 & 99.9 & 554 & 55.4 & 21.9 & 2.19 & 0.386 & 0.0418 \\
\hline $\mathrm{Cu}$ & 69.9 & 6.99 & 43.3 & 4.33 & 0.2 & 0.02 & 0.647 & 0.0647 \\
\hline $\mathrm{Fe}$ & 19000 & 1900 & 14600 & 1460 & 3.88 & 0.388 & 0.251 & 0.0251 \\
\hline $\mathrm{K}$ & 621 & 62.1 & 157 & 15.7 & 139 & 13.9 & 17.5 & 1.75 \\
\hline $\mathrm{Mg}$ & 292 & 29.2 & 225 & 22.5 & 0.228 & 0 & 0.261 & 0 \\
\hline Mn & 3580 & 358 & 2860 & 286 & $<0.0091$ & b & 0.0104 & 0 \\
\hline $\mathrm{Ni}$ & 1890 & 189 & 1450 & 145 & 2.23 & 0.255 & $<0.209$ & $\mathbf{b}$ \\
\hline$P$ & 2280 & 2280 & 2110 & 211 & 226 & 22.6 & 5.9 & 1.15 \\
\hline $\mathrm{Si}$ & 16000 & 0 & 52000 & 0 & $\mathrm{c}$ & $\mathrm{c}$ & c & c \\
\hline $\mathrm{Sr}$ & 46.7 & 47.2 & 46.6 & 43.1 & $<0.0455$ & b & $<0.0522$ & b \\
\hline Th & 10800 & 1080 & 7470 & 747 & $<0.455$ & $\mathbf{b}$ & $<0.522$ & b \\
\hline $\mathrm{U}$ & 16300 & 1630 & 13400 & 1340 & 11.5 & 1.15 & $<1.04$ & b \\
\hline $\mathrm{V}$ & 5.06 & 0.75 & 1.91 & 0.191 & 0.109 & 0.0182 & $<0.104$ & $\mathrm{~b}$ \\
\hline $\mathrm{Zn}$ & 192 & 19.2 & 108 & 10.8 & 1.25 & 0.874 & $<0.313$ & $\mathrm{~b}$ \\
\hline Radiospecies & & $g / g$ & & $3 \mathrm{~g} / \mathrm{g}$ & & $3 \mathrm{~g} / \mathrm{g}$ & & $3 \mathrm{~g} / \mathrm{g}$ \\
\hline $\mathrm{Co}-60$ & 7400 & 1200 & 5000 & 600 & 110 & 30 & 3.7 & 1.9 \\
\hline Cs-137 & 2700000 & 100000 & 1300000 & 100000 & 110000 & 10000 & 3200 & 100 \\
\hline Eu-152 & $<3200$ & $\mathrm{~b}$ & $<1600$ & $\mathrm{~b}$ & $<75$ & b & $<7.6$ & $\mathrm{~b}$ \\
\hline Eu-154 & 22000 & 3000 & 18000 & 2000 & $<52$ & b & $<6.7$ & b \\
\hline Eu-155 & 26000 & 9000 & 24000 & 5000 & $<31$ & b & $<19$ & b \\
\hline Am-241 & 150000 & 20000 & 130000 & 10000 & $<640$ & b & $<38$ & $b$ \\
\hline Gross- $\alpha$ & 260000 & 20000 & 220000 & 10000 & c & c & $\mathrm{c}$ & c \\
\hline Gross- $\beta$ & 31000000 & 1000000 & 24000000 & 1000000 & 140000 & 10000 & 4000 & 100 \\
\hline
\end{tabular}

'The symbol $<$ indicates that the concentration was below the detectable limit given by the following numerical value. 'No standard deviation for values below the detectable limit.

Not measured. 
Table 4.36. Test \#12 - percentage of each species solubilized and percentage recovery for $\mathrm{C}-104$ sludge (leach conditions: liquid:solids, $19 \mathrm{~mL} / \mathrm{g} ; \mathrm{NaOH}, 4 \mathrm{M}$; temperature, $50^{\circ} \mathrm{C}$; time, $63 \mathrm{~h}$ )

\begin{tabular}{|c|c|c|c|c|c|c|}
\hline \multirow[b]{2}{*}{ Species } & \multicolumn{2}{|c|}{$\begin{array}{c}\text { Based on residue } \\
(\%)\end{array}$} & \multicolumn{2}{|c|}{$\begin{array}{c}\text { Based on leachate \& rinse } \\
(\%) \\
\end{array}$} & \multicolumn{2}{|c|}{$\begin{array}{c}\text { Recovery } \\
(\%)\end{array}$} \\
\hline & Value & Std deviation & Value & Std deviation & Value & Std deviation \\
\hline $\mathrm{Ag}$ & -228.70 & 46.49 & 25.30 & 3.58 & 354.00 & 48.37 \\
\hline $\mathrm{Al}$ & 39.73 & 8.52 & 19.24 & 2.62 & 79.51 & 10.14 \\
\hline $\mathrm{Ba}$ & 12.87 & 12.32 & 0.00 & 0.00 & 87.13 & 12.32 \\
\hline $\mathrm{Be}$ & 59.69 & 5.70 & 43.75 & 6.19 & 84.06 & 10.30 \\
\hline $\mathrm{Ca}$ & 26.09 & 10.45 & 3.19 & 0.41 & 77.09 & 10.68 \\
\hline $\mathrm{Cd}$ & 18.46 & 11.53 & 3.20 & 0.74 & 84.74 & 11.78 \\
\hline Co & 11.40 & 12.62 & 0.00 & 0.00 & 88.60 & 12.62 \\
\hline $\mathrm{Cr}$ & 39.54 & 8.55 & 26.34 & 3.64 & 86.80 & 10.87 \\
\hline $\mathrm{Cu}$ & 32.46 & 9.55 & 32.55 & 4.39 & 100.09 & 12.43 \\
\hline $\mathrm{Fe}$ & 16.22 & 11.85 & 0.28 & 0.04 & 84.06 & 11.87 \\
\hline $\mathrm{K}$ & 72.44 & 3.90 & 345.60 & 43.95 & 373.16 & 46.23 \\
\hline $\mathrm{Mg}$ & 15.99 & 11.88 & 3.72 & 0.37 & 87.73 & 12.15 \\
\hline $\mathrm{Mn}$ & 12.90 & 12.32 & 0.01 & 0.00 & 87.11 & 12.32 \\
\hline $\mathrm{Ni}$ & 16.35 & 11.83 & 1.35 & 0.21 & 85.00 & 11.93 \\
\hline$P$ & -0.90 & 101.40 & 121.76 & 122.30 & 222.66 & 223.18 \\
\hline $\mathrm{Si}$ & -254.34 & 0.00 & a & $\mathrm{a}$ & 354.34 & 0.00 \\
\hline Sr & -8.79 & 149.05 & 0.00 & 0.00 & 108.79 & 149.05 \\
\hline Th & 24.59 & 10.66 & 0.00 & 0.00 & 75.41 & 10.66 \\
\hline $\mathrm{U}$ & 10.37 & 12.68 & 0.81 & 0.11 & 90.44 & 12.73 \\
\hline $\mathrm{V}$ & 58.85 & 7.36 & 24.68 & 5.51 & 65.84 & 11.36 \\
\hline $\mathrm{Zn}$ & 38.67 & 8.67 & 7.46 & 5.27 & 68.79 & 10.59 \\
\hline \multicolumn{7}{|c|}{ Radiospecies } \\
\hline $\mathrm{Co}-60$ & 26.33 & 14.86 & 18.61 & 5.60 & 92.28 & 18.01 \\
\hline Cs-137 & 47.51 & 4.48 & 50.43 & 4.64 & 102.93 & 6.99 \\
\hline Eu-152 & $\mathbf{b}$ & $b$ & b & $b$ & $\mathbf{b}$ & $b$ \\
\hline Eu-154 & 10.80 & 15.69 & 0.00 & 0.00 & 89.20 & 15.69 \\
\hline Eu-155 & -0.64 & 40.66 & 0.00 & 0.00 & 100.64 & 40.66 \\
\hline Am-241 & 5.51 & 14.55 & 0.00 & 0.00 & 94.49 & 14.55 \\
\hline Gross- $\alpha$ & 7.75 & 8.24 & $\mathbf{a}$ & $\mathbf{a}$ & 92.25 & 8.24 \\
\hline Gross- $\beta$ & 15.59 & 4.45 & 5.58 & 0.41 & 89.99 & 4.58 \\
\hline
\end{tabular}

"Measurement was not made.

'Insufficient data for calculation; measured concentration in original sludge was below the detectable limit or was not measured. 
Table 4.37. Test \#13 - concentrations of species in enhanced sludge washing process steps for C-104 sludge (leach conditions: liquid:solids, $27 \mathrm{~mL} / \mathrm{g} ; \mathrm{NaOH}, 4 \mathrm{M}$; temperature, $93^{\circ} \mathrm{C}$; time, $65 \mathrm{~h}$ )

\begin{tabular}{|c|c|c|c|c|c|c|c|c|}
\hline \multirow[b]{2}{*}{ Species } & \multicolumn{2}{|c|}{$\begin{array}{c}\text { Original sludge } \\
(\mu \mathrm{g} / \mathrm{g})\end{array}$} & \multicolumn{2}{|c|}{$\begin{array}{l}\text { Residual } \\
(\mu \mathrm{g} / \mathrm{g})\end{array}$} & \multicolumn{2}{|c|}{$\begin{array}{c}\text { Leachate } \\
(\mu \mathrm{g} / \mathrm{g})\end{array}$} & \multicolumn{2}{|c|}{$\begin{array}{l}\text { Rinse } \\
(\mu \mathrm{g} / \mathrm{g})\end{array}$} \\
\hline & Value & Std deviation & Value & Std deviation & Value & Std deviation & Value & Std deviation \\
\hline $\mathrm{Ag}$ & $<1.53^{\mathrm{a}}$ & b & 354 & 35.4 & 0.637 & 0.0637 & 0.366 & 0.0366 \\
\hline $\mathrm{Al}$ & 31700 & 3170 & 3920 & 392 & 2440 & 244 & 83.4 & 8.34 \\
\hline $\mathrm{Ba}$ & 120 & 12 & 132 & 13.2 & $<0.0182$ & $\mathrm{~b}$ & $<0.0222$ & $b$ \\
\hline $\mathrm{Be}$ & 34.1 & 3.41 & 21.5 & 2.15 & 1.97 & 0.197 & $<0.0111$ & $b$ \\
\hline $\mathrm{Bi}$ & 27.7 & 11.8 & 95 & 33.7 & $<0.137$ & $\mathrm{~b}$ & $<0.166$ & $b$ \\
\hline $\mathrm{Ca}$ & 1940 & 194 & 1960 & 196 & 3.81 & 0.381 & 4.79 & 0.479 \\
\hline $\mathrm{Cd}$ & 1030 & 103 & 1080 & 108 & 1.85 & 0.473 & 0.355 & 0.421 \\
\hline Co & 10.2 & 1.02 & 28.9 & 2.89 & $<0.173$ & $\mathrm{~b}$ & $<0.211$ & $\mathbf{b}$ \\
\hline $\mathrm{Cr}$ & 1130 & 113 & 378 & 37.8 & 62.8 & 6.28 & 2.15 & 0.215 \\
\hline $\mathrm{Cu}$ & 31.9 & 3.36 & 85.1 & 8.51 & 0.228 & 0.0228 & 0.122 & 0.0222 \\
\hline $\mathrm{Fe}$ & 21700 & 2170 & 21400 & 2140 & 2.94 & 0.294 & $<0.0443$ & $\mathrm{~b}$ \\
\hline $\mathrm{K}$ & 643 & 64.3 & 106 & 10.6 & 136 & 13.6 & 6.93 & 0.693 \\
\hline Mg & 387 & 48.7 & $<1.48$ & b & $<0.228$ & b & $<0.277$ & $\mathrm{~b}$ \\
\hline $\mathrm{Mn}$ & 4140 & 414 & 5720 & 572 & $<0.455$ & $\mathrm{~b}$ & $<0.0554$ & $\mathbf{b}$ \\
\hline $\mathrm{Ni}$ & 2090 & 209 & 2150 & 215 & 2.26 & 0.291 & 0.421 & 0.244 \\
\hline $\mathrm{P}$ & 77 & 104 & 141 & 14.1 & 227 & 22.7 & $<5.54$ & b \\
\hline $\mathrm{Sb}$ & 4.22 & 4.58 & $<0.888$ & $\mathrm{~b}$ & $<0.137$ & $\mathrm{~b}$ & $<0.166$ & b \\
\hline $\mathrm{Si}$ & 8500 & 0 & 13000 & 2600 & c & c & c & c \\
\hline $\mathrm{Sr}$ & 51.2 & 5.12 & 61.2 & 6.12 & $<0.0455$ & $\mathrm{~b}$ & $<0.0554$ & b \\
\hline Th & 11600 & 1160 & 11400 & 1140 & 0.61 & 0.309 & $<0.554$ & b \\
\hline $\mathrm{U}$ & 20200 & 2020 & 19400 & 1940 & 13.4 & 1.34 & $<1.11$ & $\mathrm{~b}$ \\
\hline V & 4.58 & 0.458 & 29 & 2.9 & 0.328 & 0.0728 & $<0.111$ & b \\
\hline $\mathrm{Zn}$ & 62.6 & 6.26 & 75.6 & 7.56 & 1.27 & 0.255 & $<0.332$ & $b$ \\
\hline Radiospecies & \multicolumn{2}{|c|}{$\mathrm{Ba} / \mathrm{g}$} & \multicolumn{2}{|c|}{$\mathrm{Bg} / \mathrm{g}$} & \multicolumn{2}{|c|}{$\mathrm{Ba} / \mathrm{g}$} & \multicolumn{2}{|c|}{$\mathrm{Bg} / \mathrm{g}$} \\
\hline $\mathrm{Co}-60$ & 6100 & 3700 & 9000 & 2300 & $\mathrm{c}$ & c & c & $\mathrm{c}$ \\
\hline Cs-137 & 2700000 & 100000 & 760000 & 20000 & 210000 & 10000 & 7800 & 100 \\
\hline $\mathrm{Eu}-152$ & $<4700$ & b & c & c & c & c & c & c \\
\hline Eu-154 & 18000 & 8000 & 34000 & 8000 & c & c & $c$ & c \\
\hline Eu-155 & 23000 & 25000 & 21000 & 12000 & $\mathrm{c}$ & c & c & $\mathrm{c}$ \\
\hline $\mathrm{Pu}-238$ & 9300 & 100 & 16000 & 1000 & c & c & $\mathrm{c}$ & c \\
\hline $\mathrm{Pu}-239 / 240$ & 59000 & 1000 & 94000 & 2000 & $\mathrm{c}$ & c & c & c \\
\hline Pu-all & c & c & 110000 & 10000 & c & c & c & c \\
\hline Am-241 & 86000 & 49000 & 210000 & 30000 & c & c & c & c \\
\hline Gross- $\alpha$ & 210000 & 10000 & 320000 & 10000 & c & $c$ & $\mathrm{c}$ & c \\
\hline Gross- $\beta$ & 30000000 & 1000000 & 34000000 & 1000000 & 250000 & 10000 & 9300 & 100 \\
\hline
\end{tabular}

The symbol < indicates that the concentration was below the detectable limit given by the following numerical value. 'No standard deviation for values below the detectable limit.

'Not measured. 
Table 4.38. Test \#13 - percentage of each species solubilized and percentage recovery for $C$-104 sludge (leach conditions: liquid:solids, $27 \mathrm{~mL} / \mathrm{g} ; \mathrm{NaOH}, 4 \mathrm{M}$; temperature, $93^{\circ} \mathrm{C}$; time, $65 \mathrm{~h}$ )

\begin{tabular}{|c|c|c|c|c|c|c|}
\hline \multirow[b]{2}{*}{ Species } & \multicolumn{2}{|c|}{$\begin{array}{c}\text { Based on residue } \\
(\%) \\
\end{array}$} & \multicolumn{2}{|c|}{$\begin{array}{c}\text { Based on leachate \& rinse } \\
(\%)\end{array}$} & \multicolumn{2}{|c|}{$\begin{array}{c}\text { Recovery } \\
(\%)\end{array}$} \\
\hline & Value & Std deviation & Value & Std deviation & Value & Std deviation \\
\hline $\mathrm{Ag}$ & $\mathrm{a}$ & a & a & a & $\mathrm{a}$ & a \\
\hline Al & 90.23 & 1.38 & 83.16 & 11.38 & 92.92 & 12.15 \\
\hline $\mathrm{Ba}$ & 13.13 & 12.29 & 0.00 & 0.00 & 86.87 & 12.29 \\
\hline $\mathrm{Be}$ & 50.21 & 7.04 & 58.18 & 8.23 & 107.97 & 13.24 \\
\hline $\mathrm{Bi}$ & -170.84 & 150.14 & 0.00 & 0.00 & 270.84 & 150.14 \\
\hline $\mathrm{Ca}$ & 20.21 & 11.28 & 7.28 & 0.92 & 87.06 & 11.82 \\
\hline Cd & 17.19 & 11.71 & 2.55 & 1.02 & 85.35 & 11.93 \\
\hline Co & -123.75 & 31.64 & 0.00 & 0.00 & 223.75 & 31.64 \\
\hline $\mathrm{Cr}$ & 73.58 & 3.74 & 60.05 & 8.22 & 86.47 & 10.64 \\
\hline $\mathrm{Cu}$ & -110.67 & 30.60 & 15.41 & 2.32 & 226.08 & 31.84 \\
\hline $\mathrm{Fe}$ & 22.12 & 11.01 & 0.14 & 0.02 & 78.02 & 11.02 \\
\hline $\mathrm{K}$ & 86.98 & 1.84 & 236.12 & 31.88 & 249.14 & 32.88 \\
\hline $\mathrm{Mg}$ & 100.00 & 0.00 & 0.00 & 0.00 & 0.00 & 0.00 \\
\hline Mn & -9.11 & 15.43 & 0.00 & 0.00 & 109.11 & 15.43 \\
\hline $\mathrm{Ni}$ & 18.76 & 11.49 & 1.52 & 0.32 & 82.76 & 11.60 \\
\hline$P$ & -44.61 & 195.85 & 2968.72 & 4020.67 & 3113.33 & 4215.50 \\
\hline $\mathrm{Sb}$ & 100.00 & 0.00 & 0.00 & 0.00 & 0.00 & 0.00 \\
\hline $\mathrm{Si}$ & -20.78 & 24.16 & $\mathrm{~b}$ & b & 120.78 & 24.16 \\
\hline SI & 5.60 & 13.35 & 0.00 & 0.00 & 94.40 & 13.35 \\
\hline Th & 22.39 & 10.98 & 0.05 & 0.03 . & 77.66 & 10.98 \\
\hline $\mathrm{U}$ & 24.16 & 10.73 & 0.67 & 0.09 & 76.51 & 10.77 \\
\hline $\mathrm{V}$ & -400.04 & 70.72 & 72.12 & 17.56 & 572.16 & 77.65 \\
\hline $\mathrm{Zn}$ & 4.63 & 13.49 & 20.43 & 4.58 & 115.80 & 15.55 \\
\hline \multicolumn{7}{|l|}{ Radiospecies } \\
\hline Co-60 & -16.52 & 76.69 & b & b & 116.52 & 76.69 \\
\hline Cs-137 & 77.77 & 1.01 & 84.52 & 4.87 & 106.75 & 5.47 \\
\hline Eu-152 & $\mathbf{a}$ & $\mathrm{a}$ & a & a & $\mathbf{a}$ & $\mathbf{a}$ \\
\hline Eu-154 & -49.17 & 75.02 & b & b & 149.17 & 75.02 \\
\hline Eu-155 & 27.90 & 88.55 & b & b & 72.10 & 88.55 \\
\hline Pu-238 & -35.87 & 8.62 & b & b & 135.87 & 8.62 \\
\hline $\mathrm{Pu}-239 / 240$ & -25.82 & 3.42 & $\mathrm{~b}$ & b & 125.82 & 3.42 \\
\hline Pu-all & -27.75 & 11.76 & $\mathrm{~b}$ & $\mathrm{~b}$ & 127.75 & 11.76 \\
\hline Am-24l & -92.84 & 113.27 & b & $b$ & 192.84 & 113.27 \\
\hline Gross- $\alpha$ & -20.34 & 6.85 & b & $b$ & 120.34 & 6.85 \\
\hline Gross- $\beta$ & 10.50 & 3.98 & 9.06 & 0.45 & 98.56 & 4.22 \\
\hline
\end{tabular}

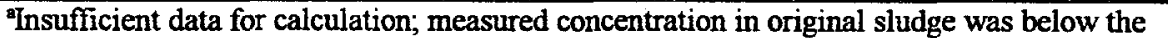
detectable limit or was not measured.

bMeasurement was not made. 
Table 4.39. Effect of operating parameters on caustic leaching of C-104 sludge

\begin{tabular}{cccccccccc}
\hline & Liquid:solid & $\mathrm{NaOH}$ & $\mathrm{T}$ & $\mathrm{Time}$ & \multicolumn{4}{c}{ \% Removal } \\
\cline { 7 - 10 } Test & $(\mathrm{mL} / \mathrm{g})$ & $(M)$ & $\left({ }^{\circ} \mathrm{C}\right)$ & $(\mathrm{h})$ & $\mathrm{Al}$ & $\mathrm{Cr}$ & $\mathrm{U}$ & $\mathrm{Cs}$ \\
\hline 11 & 31 & 3.99 & 80 & 65 & 28.7 & 59.5 & 17.1 & 64.4 \\
12 & 19 & 3.99 & 50 & 63 & 39.7 & 39.5 & 10.4 & 47.5 \\
13 & 27 & 3.99 & 93 & 65 & 90.2 & 73.6 & 24.2 & 77.8 \\
\hline
\end{tabular}

Table 4.40. Concentration of selected constituents in dry, untreated C-104 sludge determined by different researchers

\begin{tabular}{ccccc}
\hline Species & Unit of measure & LANL $^{2}$ & ORNL $^{\mathrm{b}}$ & ORNL $^{\mathrm{c}}$ \\
\hline $\mathrm{Al}$ & $\mu \mathrm{g} / \mathrm{g}$ & 63200. & 86800 & 63900. \\
$\mathrm{Cr}$ & $\mu \mathrm{g} / \mathrm{g}$ & 2280 & 3100 & 1770. \\
$\mathrm{P}$ & $\mu \mathrm{g} / \mathrm{g}$ & 6320. & 211. & 4050. \\
$\mathrm{U}$ & $\mu \mathrm{g} / \mathrm{g}$ & 30000. & 55300. & 29000. \\
$\mathrm{Cs}-137$ & $\mu \mathrm{Ci} / \mathrm{g}$ & 174. & 200. & 130. \\
$\mathrm{Pu}-239 / 240$ & $\mu \mathrm{Ci} / \mathrm{g}$ & 4.93 & 4.4 & \\
\hline
\end{tabular}

${ }^{\mathrm{a}}$ Temer and Villarreal, LAUR 97-2889 (1997).

'This work, high-water-content sample.

'This work, low-water-content sample.

Table 4.41. Comparison of percentage of selected species removed from C-104 sludge by different researchers

\begin{tabular}{cccc}
\hline & \multicolumn{3}{c}{ \% Removed } \\
\cline { 2 - 4 } Species & LANL $^{*}$ & ORNL $^{\mathrm{b}}$ & ORNL $^{\mathrm{c}}$ \\
\hline $\mathrm{Al}$ & 97 & 40 & 90 \\
$\mathrm{Cr}$ & 52 & 40 & 74 \\
$\mathrm{P}$ & 89 & & \\
$\mathrm{U}$ & 4 & 10 & 24 \\
$\mathrm{Cs}$ & 100 & 48 & 78 \\
$\mathrm{Pu}$ & 0 & & \\
\hline
\end{tabular}

${ }^{\mathrm{B}}$ Temer and Villarreal, LAUR 97-2889 (1997); first leach $2.7 \mathrm{M} \mathrm{NaOH}, 5 \mathrm{~h}$, $100^{\circ} \mathrm{C}, 5 \mathrm{wt} \%$ solids; second leach $3.2 \mathrm{M} \mathrm{NaOH}, 5 \mathrm{~h}, 100^{\circ} \mathrm{C}, 1 \mathrm{wt} \%$ solids.

${ }^{\circ}$ This work; $3.99 \mathrm{M} \mathrm{NaOH}, 63 \mathrm{~h}, 50^{\circ} \mathrm{C}$, and liquid:solids of 19:1.

'This work; $3.99 \mathrm{M} \mathrm{NaOH}, 65 \mathrm{~h}, 93^{\circ} \mathrm{C}$, and liquid:solids of 27:1. 


\subsection{SLUDGE S-101}

One experiment was performed to measure the effect of the enhanced sludge washing process on sludge from tank S-101. Analyses of the untreated sludge and the phases into which it partitioned are shown in Table 4.42. Major constituents in this sludge are aluminum, silicon, and uranium. The sludge was leached with $4 \mathrm{MNaOH}$ at $93^{\circ} \mathrm{C}$ with a liquid:solids ratio of $16 \mathrm{~mL} / \mathrm{g}$ for $65 \mathrm{~h}$. Calculated values of the percentage of each species leached from the sludge and total recovery of each species are listed in Table 4.43. About $98 \%$ of the aluminum and $99 \%$ of the cesium were leached from the sludge, and the excellent material balances (percentage recovery) indicate that these values are reliable. The data indicate that approximately $86 \%$ of the chromium, $26 \%$ of the uranium, and $19 \%$ of the plutonium were removed from the sludge, although the uncertainty is greater than that of the aluminum and cesium data.

The concentrations of selected species in sludge S-101 quantified in this work are compared with measurements made by Lumetta et al. (1997b) in Table 4.44. Agreement is quite good for aluminum, chromium, uranium, cesium-137, and plutonium-239/240. However, there is a great difference in the measured concentration of phosphorus. In their leaching study, Lumetta et al. (1997b) used a lower caustic concentration $(\sim 2.5 M)$ with a slightly higher temperature $\left(100^{\circ} \mathrm{C}\right)$ and about twice the leaching time $(100 \mathrm{~h})$ than was used in this study. The percentages of aluminum, chromium, and cesium-137 removed from the sludge in the two studies are similar, as shown in Table 4.45. There is significant disagreement in the measured behavior of uranium and plutonium-239/240. However, part of the difference may be attributable to the substantial standard deviations and slightly low recoveries estimated in the present study (Table 4.43). 
Table 4.42. Test \#14 - concentrations of species in enhanced sludge washing process steps for S-101 sludge (leach conditions: liquid:solids, $16 \mathrm{~mL} / \mathrm{g} ; \mathrm{NaOH}, 4 \mathrm{M}$; temperature, $93^{\circ} \mathrm{C}$; time, $65 \mathrm{~h}$ )

\begin{tabular}{|c|c|c|c|c|c|c|c|c|}
\hline \multirow[b]{2}{*}{ Species } & \multicolumn{2}{|c|}{$\begin{array}{c}\text { Original sludge } \\
(\mu \mathrm{g} / \mathrm{g})\end{array}$} & \multicolumn{2}{|c|}{$\begin{array}{c}\text { Residual } \\
(\mu \mathrm{g} / \mathrm{g})\end{array}$} & \multicolumn{2}{|c|}{$\begin{array}{c}\text { Leachate } \\
(\mu \mathrm{g} / \mathrm{g})\end{array}$} & \multicolumn{2}{|c|}{$\begin{array}{l}\text { Rinse } \\
(\mu \mathrm{g} / \mathrm{g})\end{array}$} \\
\hline & Value & Std deviation & Value & Std deviation & Value & Std deviation & Value & Std deviation \\
\hline $\mathrm{Ag}$ & 9.04 & 0.904 & 24 & 2.4 & 0.186 & 0.0186 & 0.224 & 0.0224 \\
\hline $\mathrm{Al}$ & 86300 & 8630 & 8760 & 876 & 7050 & 705 & 106 & 10.6 \\
\hline $\mathrm{Ba}$ & 44.9 & 4.49 & 144 & 14.4 & $<0.0178^{\mathrm{a}}$ & $b$ & $<0.0213$ & $b$ \\
\hline $\mathrm{Be}$ & $<0.171$ & $\mathrm{~b}$ & $<0.238$ & $\mathbf{b}$ & $<0.00888$ & $\mathrm{~b}$ & $<0.0107$ & $\mathrm{~b}$ \\
\hline $\mathrm{Bi}$ & 17.9 & 1.79 & 457 & 45.7 & 0.277 & 0.0277 & $<0.160$ & $\mathrm{~b}$ \\
\hline $\mathrm{Ca}$ & 1330 & 133 & 1600 & 160 & 3.88 & 0.388 & 1.66 & 0.166 \\
\hline $\mathrm{Cd}$ & 14.3 & 10.9 & 27.9 & 6.67 & 0.959 & 0.16 & 0.448 & 0.405 \\
\hline Co & 4.94 & 0.495 & 16.7 & 1.67 & $<0.169$ & $\mathrm{~b}$ & $<0.203$ & $\mathbf{b}$ \\
\hline $\mathrm{Cr}$ & 3430 & 343 & 2220 & 222 & 365 & 36.5 & 5.85 & 0.585 \\
\hline $\mathrm{Cu}$ & 52.6 & 5.26 & 77.4 & 7.74 & 2.91 & 0.291 & 0.128 & 0.0128 \\
\hline $\mathrm{Fe}$ & 1830 & 183 & 5920 & 592 & 10.1 & 1.01 & $<0.0426$ & $b$ \\
\hline $\mathrm{K}$ & 839 & 83.9 & $<23.8$ & $\mathrm{~b}$ & 162 & 16.2 & 4.82 & 0.482 \\
\hline $\mathrm{Mg}$ & 31.7 & 3.17 & 67.4 & 15.2 & $<0.222$ & $\mathrm{~b}$ & $<0.267$ & b \\
\hline $\mathrm{Mn}$ & 1600 & 160 & 7330 & 733 & $<0.444$ & $\mathrm{~b}$ & $<0.0533$ & $b$ \\
\hline $\mathrm{Ni}$ & 116 & 11.6 & 491 & 49.1 & 0.435 & 0.0888 & 0.267 & 0.533 \\
\hline $\mathbf{P}$ & $<85.0$ & $\mathbf{b}$ & 1680 & 168 & 20.3 & 2.03 & $<5.33$ & $\mathrm{~b}$ \\
\hline $\mathrm{Sb}$ & $<.56$ & $b$ & $<3.57$ & $\mathrm{~b}$ & $<0.133$ & $\mathrm{~b}$ & $<0.160$ & $b$ \\
\hline $\mathrm{Si}$ & 9200 & 1840 & 29000 & 5800 & c & c & c & c \\
\hline $\mathrm{Sr}$ & 404 & 40.4 & 1220 & 122 & 0.0799 & 0.00799 & $<0.0533$ & $\mathrm{~b}$ \\
\hline Th & 240 & 24 & 824 & 82.4 & $<0.444$ & b & $<0.533$ & $b$ \\
\hline $\mathrm{U}$ & 7420 & 742 & 24700 & 2470 & 2.98 & 0.426 & $<1.07$ & $b$ \\
\hline V & 9.73 & 0.973 & 22.4 & 2.24 & 0.408 & 0.0408 & $<0.107$ & b \\
\hline $\mathrm{Zn}$ & 205 & 20.5 & 154 & 15.4 & 0.506 & 0.0506 & $<0.320$ & b \\
\hline Radiospecies & \multicolumn{2}{|c|}{$\mathrm{Bo} / \mathrm{g}$} & \multicolumn{2}{|c|}{$\mathrm{Bg} / \mathrm{g}$} & \multicolumn{2}{|c|}{$\mathrm{Bq} / \mathrm{g}$} & \multicolumn{2}{|r|}{$\mathrm{Bq} / \mathrm{g}$} \\
\hline $\mathrm{Co}-60$ & c & $\mathrm{c}$ & 1500 & 700 & c & $\mathrm{c}$ & c & $\mathrm{c}$ \\
\hline Cs-137 & 3800000 & 100000 & 230000 & 10000 & 340000 & 10000 & 5400 & 100 \\
\hline Eu-152 & c & c & c & c & c & c & c & $\mathbf{c}$ \\
\hline Eu-154 & c & c & 17000 & 3000 & c & c & c & c \\
\hline Eu-155 & c & $\mathrm{c}$ & 9300 & 7300 & c & c & c & c \\
\hline $\mathrm{Pu}-238$ & 260 & 20 & 840 & 30 & c & c & c & c \\
\hline $\mathrm{Pu}-239 / 240$ & 9600 & 600 & 35000 & 1000 & c & $\mathrm{c}$ & c & c \\
\hline Pu-all & 9800 & 600 & 35000 & 1000 & c & c & c & c \\
\hline Am-241 & c & c & 21000 & 18000 & c & c & c & c \\
\hline Gross- $\alpha$ & 15000 & 1000 & 57000 & 3000 & c & c & c & $\mathrm{c}$ \\
\hline Gross- $\beta$ & 29000000 & 1000000 & 81000000 & 1000000 & 410000 & 10000 & 6400 & 100 \\
\hline
\end{tabular}

The symbol $<$ indicates that the concentration was below the detectable limit given by the following numerical value. 'No standard deviation for values below the detectable limit.

Not measured. 
Table 4.43. Test \#14 - percentage of each species solubilized and percentage recovery for S-101 sludge (leach conditions: liquid:solids, $16 \mathrm{~mL} / \mathrm{g}$, NaOH, $4 \mathrm{M}$; temperature, $93^{\circ} \mathrm{C}$; time, $65 \mathrm{~h}$ )

\begin{tabular}{|c|c|c|c|c|c|c|}
\hline \multirow[b]{2}{*}{ Species } & \multicolumn{2}{|c|}{$\begin{array}{c}\text { Based on residue } \\
(\%)\end{array}$} & \multicolumn{2}{|c|}{$\begin{array}{c}\text { Based on leachate \& rinse } \\
(\%)\end{array}$} & \multicolumn{2}{|c|}{$\begin{array}{c}\text { Recovery } \\
(\%) \\
\end{array}$} \\
\hline & Value & Std deviation & Value & Std deviation & Value & Std deviation \\
\hline $\mathrm{Ag}$ & 41.31 & 8.30 & 85.16 & 10.77 & 143.85 & 16.87 \\
\hline $\mathrm{Al}$ & 97.76 & 0.32 & 97.29 & 13.55 & 99.54 & 13.71 \\
\hline $\mathrm{Ba}$ & 29.11 & 10.03 & 0.00 & 0.00 & 70.89 & 10.03 \\
\hline $\mathrm{Be}$ & $a$ & $\mathrm{a}$ & $\mathrm{a}$ & a & $a$ & $a$ \\
\hline $\mathrm{Bi}$ & -464.36 & 79.81 & 17.85 & 2.52 & 582.22 & 81.10 \\
\hline $\mathrm{Ca}$ & 73.41 & 3.76 & 6.46 & 0.79 & 33.05 & 4.27 \\
\hline $\mathrm{Cd}$ & 56.87 & 34.45 & 155.03 & 138.06 & 198.16 & 167.38 \\
\hline $\mathrm{Co}$ & 25.27 & 10.58 & 0.00 & 0.00 & 74.73 & 10.58 \\
\hline $\mathrm{Cr}$ & 85.69 & 2.02 & 127.00 & 17.67 & 141.30 & 18.78 \\
\hline $\mathrm{Cu}$ & 67.47 & 4.60 & 69.86 & 9.48 & 102.39 & 12.51 \\
\hline $\mathrm{Fe}$ & 28.49 & 10.11 & 6.37 & 0.90 & 77.88 & 10.59 \\
\hline $\mathrm{K}$ & 100.00 & 0.00 & 237.00 & 32.56 & 237.00 & 32.56 \\
\hline $\mathrm{Mg}$ & 53.00 & 11.59 & 0.00 & 0.00 & 47.00 & 11.59 \\
\hline $\mathrm{Mn}$ & -1.27 & 14.32 & 0.00 & 0.00 & 101.27 & 14.32 \\
\hline $\mathrm{Ni}$ & 6.43 & 13.23 & 10.03 & 11.47 & 103.60 & 18.04 \\
\hline $\mathrm{P}$ & $\mathrm{a}$ & a & a & $\mathbf{a}$ & $\mathbf{a}$ & $\mathrm{a}$ \\
\hline Sb & a & a & a & a & a & $\mathbf{a}$ \\
\hline Si & 30.32 & 19.71 & 0.00 & 0.00 & 69.68 & 19.71 \\
\hline $\mathrm{Sr}$ & 33.25 & 9.44 & 0.23 & 0.03 & 66.98 & 9.46 \\
\hline Th & 24.11 & 10.73 & 0.00 & 0.00 & 75.89 & 10.73 \\
\hline $\mathrm{U}$ & 26.42 & 10.41 & 0.46 & 0.08 & 74.05 & 10.44 \\
\hline $\mathrm{V}$ & 49.11 & 7.20 & 48.38 & 6.84 & 99.27 & 12.16 \\
\hline $\mathrm{Zn}$ & 83.39 & 2.35 & 2.85 & 0.40 & 19.45 & 2.57 \\
\hline \multicolumn{7}{|l|}{ Radiospecies } \\
\hline $\mathrm{Co}-60$ & a & $\mathbf{a}$ & $\mathbf{a}$ & $\mathbf{a}$ & $\mathbf{a}$ & a \\
\hline Cs-137 & 98.66 & 0.07 & 106.75 & 4.14 & 108.09 & 4.16 \\
\hline Eu-152 & $\mathbf{a}$ & $\mathbf{a}$ & $\mathbf{a}$ & $\mathbf{a}$ & $\mathbf{a}$ & $\mathrm{a}$ \\
\hline Eu-154 & $\mathbf{a}$ & $\mathbf{a}$ & $\mathbf{a}$ & $\mathbf{a}$ & $\mathbf{a}$ & a \\
\hline Eu-155 & $\mathbf{a}$ & $\mathbf{a}$ & a & $\mathbf{a}$ & a & $\mathbf{a}$ \\
\hline $\mathrm{Pu}-238$ & 28.58 & 6.06 & $\mathrm{~b}$ & $b$ & 71.42 & 6.06 \\
\hline Pu-239/240 & 19.41 & 5.54 & b & $b$ & 80.59 & 5.54 \\
\hline Pu-all & 21.05 & 5.33 & $b$ & $\mathrm{~b}$ & 78.95 & 5.33 \\
\hline Am-241 & a & a & a & $\mathrm{a}$ & $\mathbf{a}$ & a \\
\hline Gross- $\alpha$ & 16.00 & 7.13 & b & b & 84.00 & 7.13 \\
\hline Gross- $\beta$ & 38.26 & 2.26 & 16.86 & 0.70 & 78.60 & 2.84 \\
\hline
\end{tabular}

Insufficient data for calculation; measured concentration in original sludge was below the detectable limit or was not measured.

Measurement was not made. 
Table 4.44. Concentration of selected constituents in dry, untreated S-101 sludge determined by different researchers

\begin{tabular}{cccc}
\hline Species & Unit of measure & PNNL $^{\mathrm{a}}$ & ORNL $^{\mathrm{b}}$ \\
\hline $\mathrm{Al}$ & $\mu \mathrm{g} / \mathrm{g}$ & 147000. & 127000. \\
$\mathrm{Cr}$ & $\mu \mathrm{g} / \mathrm{g}$ & 7110. & 5030. \\
$\mathrm{P}$ & $\mu \mathrm{g} / \mathrm{g}$ & 2300. & $<125$. \\
$\mathrm{U}$ & $\mu \mathrm{g} / \mathrm{g}$ & 9560. & 10900. \\
$\mathrm{Cs}-137$ & $\mu \mathrm{Ci} / \mathrm{g}$ & 138. & 151. \\
$\mathrm{Pu}-239 / 240$ & $\mu \mathrm{Ci} / \mathrm{g}$ & 0.48 & 0.38 \\
\hline
\end{tabular}

"Lumetta et al., PNNL-11636 (1997b).

'This work.

Table 4.45. Comparison of percentage of selected species removed from S-101 sludge by different researchers

\begin{tabular}{|c|c|c|}
\hline \multirow[b]{2}{*}{ Species } & \multicolumn{2}{|c|}{ \% Removed } \\
\hline & $\mathrm{PNNL}^{\mathrm{a}}$ & $\mathrm{ORNL}^{\mathrm{b}}$ \\
\hline $\mathrm{Al}$ & 96 & 98 \\
\hline $\mathrm{Cr}$ & 89 & 86 \\
\hline $\mathbf{P}$ & 97 & \\
\hline $\mathrm{U}$ & 1 & 26 \\
\hline Cs & 100 & 99 \\
\hline $\mathrm{Pu}$ & $<4$ & 19 \\
\hline
\end{tabular}

${ }^{a}$ Lumetta et al., PNNL-11636 (1997b); first leach $2.5 \mathrm{M}$ $\mathrm{NaOH}, 5 \mathrm{~h}, 100^{\circ} \mathrm{C}, 5 \mathrm{wt} \%$ solids; second leach $2.7 \mathrm{MNaOH}$, $100 \mathrm{~h}, 100^{\circ} \mathrm{C}, 1 \mathrm{wt} \%$ solids.

${ }^{\circ}$ This work; $3.99 \mathrm{M} \mathrm{NaOH}, 65 \mathrm{~h}, 93^{\circ} \mathrm{C}$, and liquid:solids of $16: 1$. 


\section{SUMMARY AND RECOMMENDATIONS}

\subsection{SUMMARY}

Fourteen separate experiments were performed to characterize the behavior of six different Hanford sludges under different caustic leaching conditions. Six tests were performed with sludge from tank S-104, two tests on SX-113, one test on C-105, one test on C-107, three tests on C-104, and one test on S-101. The test variables were leaching time, leaching temperature, sodium hydroxide concentration in the leach solution, volume of the leaching solution, and mass of the sludge.

Sludge S-104 was leached with sodium hydroxide solutions ranging in concentration from 3.8 to $6.33 \mathrm{M}$ for durations ranging from 4 to $126 \mathrm{~h}$. More than $95 \%$ of the chromium and cesium were removed under all of the conditions tested. Removal of aluminum improved with increased caustic concentration and increased leaching time, ranging from 20 to $96 \%$ removal. Temperature was varied over a range of 67 to $80^{\circ} \mathrm{C}$, and the percentage of metals removed from the sludge increased with increasing temperature.

Increasing both leaching time and temperature increased the quantities of aluminum, chromium, and cesium removed from the SX-113 sludge with $6.33 \mathrm{MNaOH}$. Removal of aluminum ranged from 51 to $79 \%$; removal of chromium ranged from 53 to $66 \%$; and cesium removal ranged from 60 to $86 \%$.

Approximately $97 \%$ of the aluminum and $71 \%$ of the chromium were removed from sludge C-105 with $6.33 \mathrm{M} \mathrm{NaOH}$ at $70^{\circ} \mathrm{C}$ in $22 \mathrm{~h}$. About $76 \%$ of the cesium was also removed. Similar conditions resulted in the removal of $82 \%$ of the aluminum, $70 \%$ of the chromium, and $73 \%$ of the cesium from sludge C-107.

Leaching tests with sludge C-104 were performed at a sodium hydroxide concentration of $4 M$ and leaching times of $63-65 \mathrm{~h}$. As operating temperature was increased from 50 to $93^{\circ} \mathrm{C}$, the amount 
of aluminum removed increased from 29 to $90 \%$ and chromium removal increased from 40 to $74 \%$. Removal of cesium also increased with temperature.

Sludge S-101 was leached with $4 \mathrm{MNaOH}$ at $93^{\circ} \mathrm{C}$ for $65 \mathrm{~h}$. About $98 \%$ of the aluminum, $86 \%$ of the chromium, and $99 \%$ of the cesium were removed from the sludge.

Analyses of the untreated sludges reported by various researchers were compared with the results obtained in this work. Generally there was good agreement on concentrations of aluminum, chromium, and cesium. One exception was sludge $\mathrm{C}-107$, where there was considerable scatter in the data. There was uniform disagreement on the phosphorus concentration. It is postulated that some differences can be attributed to variations in the nonhomogeneous samples, but differences in phosphorus results appear to be an artifact of the analysis technique. Comparisons of the removal of selected metals from the sludge exhibited about the same level of agreement.

\subsection{RECOMMENDATIONS}

Planned parametric studies of the effect of caustic concentration, liquid:solids ratio, and temperature on the enhanced sludge washing process should also evaluate the effect of leaching time. The data presented in this report can aid in selecting conditions and duration of the process.

Because sludge samples from one tank may be distributed to more than one researcher, it is recommended that a large sample be collected and homogenized with strong mechanical agitation prior to distribution of aliquots. This would eliminate the concern that some differences in results could occur because of sample inhomogeneity.

The chemical analyses of sludges and process streams continue to be a problem and need to be improved. The methods to analyze phosphorus should be reviewed to determine the cause of the large differences reported by various workers. 


\section{REFERENCES}

Beahm, E. C., Weber, C. F., Dillow, T. A., Bush, S. A., Lee, S. Y., and Hunt, R. D. June 1997. Sludge Treatment Studies, ORNL/TM-13371, Lockheed Martin Energy Research, Oak Ridge National Laboratory.

Brooks, K. P, Philips, J. R., Meyers, R. L., Rappe, K. G., Rector, D. R., and Smith, P. A. September 1996. "TWRS Low-Level Waste Addback Program: Sludge Pretreatment Studies Using Hanford Tank C-107," Letter Report prepared for Westinghouse Hanford Company, Battelle, Pacific Northwest Laboratory.

Collins, J. L., Egan, B. Z., Beahm, E. C., Chase, C. W., and Anderson, K. K. August 1997. Characterization and Leaching Study of Sludge from Melton Valley Storage Tank W-25, ORNL/TM-13445, Lockheed Marietta Energy Research, Oak Ridge National Laboratory.

Colton, N. G. September 1996. Status Report: Pretreatment Chemistry Evaluation-Wash and Leach Factors for the Single-shell Tank Waste Inventory, PNNL-1 1290, Battelle, Pacific Northwest National Laboratory.

Colton, N. G. August 1997. Status Report: Pretreatment Chemistry Evaluation FY 1997 - Wash and Leach Factors for the Single-shell Tank Waste Inventory, PNNL-11646, Battelle, Pacific Northwest National Laboratory.

Holman, J. P. 1971. Experimental Methods for Engineers, 2nd ed., McGraw-Hill, New York.

Lumetta, G. J., Rapko, B. M., Wagner, M. J., Liu, J., and Chen, Y. L. August 1996. Washing and Caustic Leaching of Hanford Tank Sludges: Results of FY 1996 Studies, PNNL-11278, Battelle, Pacific Northwest National Laboratory.

Lumetta, G. J., Rapko,B. M., Temer, D. J., and Egan, B. Z. September 1997a. "Enhanced Sludge Washing for Pretreating Hanford Tank Sludges," 214th ACS National Meeting, Division of Industrial and Engineering Chemistry, Las Vegas, September 13-17, 1997.

Lumetta, G. J., Burgeson, I. E., Wagner, M. J., Liu, J., and Chen, Y. L. August 1997b. Washing and Caustic Leaching of Hanford Tank Sludge: Results of FY 1997 Studies, PNNL-11636, Battelle, Pacific Northwest National Laboratory.

Keller, J. M., Giaquinto, J. M., and Meeks, A. M. December 1996. Characterization of the MVST Waste Tanks Located at ORNL, ORNL/TM-13357, Lockheed Martin Energy Research, Oak Ridge National Laboratory.

Rapko, B. M., Lumetta, G. J., and Wagner, M. J. August 1995. Washing and Caustic Leaching of Hanford Tank Sludges: Results of FY 1995 Studies, PNL-10712, Battelle, Pacific Northwest Laboratory.

Rapko, B. M., Blanchard, D. L., Colton, N. G., Felmy, A. R., Liu, J., and Lumetta, G. J. March 1996. The Chemistry of Sludge Washing and Caustic Leaching Processes for Selected Hanford Tank Wastes, PNNL-11089, Battelle, Pacific Northwest National Laboratory. 
Rapko, B. M., Delegard, C. H., and Wagner, M. J. August 1997. Oxidative Dissolution of Chromium from Hanford Tank Sludges Under Alkaline Conditions, PNNL-1 1571, Battelle, Pacific Northwest National Laboratory.

Sears, M. B., Botts, J. L., Ceo, R. N., Ferrada, J. J., Griest, W. H., Keller, J. M., and Schenley, R. L. September 1990. Sampling and Analysis of Radioactive Liquid Wastes and Sludges in the Melton Valley and Evaporator Facility Storage Tanks at ORNL, ORNL/TM-1 1652, Lockheed Martin Energy Systems, Oak Ridge National Laboratory.

Slaathaug, E. J. July 1995. Tri-Party Agreement Alternative Engineering Data Package for the Tank Waste Remediation System Environmental Impact Statement, WHC-SD-WM-EV-104, Westinghouse Hanford Company.

Temer, D. J., and Villarreal, R. August 1996. Sludge Washing and Alkaline Leaching Tests on Actual Hanford Tank Sludge: FY 1996 Results, LAUR 96-2839, Los Alamos National Laboratory.

Temer, D. J., and Villarreal, R. August 1997. Sludge Washing and Alkaline Leaching Tests on Actual Hanford Tank Sludge: FY 1997 Results, LAUR 97-2889, Los Alamos National Laboratory.

Ulrich, C., Fox, E., and Shotton, K. October 1995. User's Manual: SigmaPlot Scientific Graphing Software, Rev. 3, Jandel Corporation, USA.

Weber, C. F., and Beahm, E. C. October 1996. Chemical Modeling of Waste Sludges, ORNL/TM-13200, Lockheed Martin Energy Research, Oak Ridge National Laboratory. 
<smiles>C1CCCC1</smiles> 


\section{INTERNAL DISTRIBUTION}

$\begin{aligned} \text { 1. } & \text { K. K. Anderson } \\ 2 . & \text { E. C. Beahm } \\ 3-4 . & \text { C. W. Chase } \\ 5 . & \text { E. D. Collins } \\ 6-7 . & \text { J. L. Collins } \\ 8 . & \text { A. G. Croff } \\ 9 . & \text { J. N. Herndon } \\ 10-14 . & \text { R. D. Hunt } \\ 15-16 . & \text { R. T. Jubin } \\ 17 . & \text { J. M. Keller } \\ 18 . & \text { L. N. Klatt } \\ 19 . & \text { C. P. McGinnis }\end{aligned}$

\author{
20. L. E. McNeese \\ 21. T. E. Myrick \\ 22. K. E. Plummer \\ 23. S. M. Robinson \\ 24-33. B. B. Spencer \\ 34. J. S. Watson \\ 35. C. F. Weber \\ 36. T. D. Welch \\ 37. ORNL Laboratory Records, RC \\ 38. Central Research Library \\ 39. Laboratory Records (OSTI)
}

\section{EXTERNAL DISTRIBUTION}

40. N. G. Colton, Pacific Northwest National Laboratory, Battelle Boulevard, P.O. Box 999, MS K8-93, Richland, Washington 99352

41. B. Z. Egan, 103 Lewis Lane, Oak Ridge, Tennessee 37830

42. J. L. Harness, Department of Energy, Oak Ridge Operations, P.O. Box 2001, Oak Ridge, Tennessee 37831-8620

43. R. A. Kirkbride, Numatec Hanford Corporation, P.O. Box 1300, MS H5-27, Richland, Washington 99352

44. Louis Kovach, Hanford, P.O. Box 1970, K6-51, Richland, Washington 99352

45. W. L. Kuhn, Pacific Northwest National Laboratory, Battelle Boulevard, P.O. Box 999, MS K8-93, Richland, Washington 99352

46. J. P. LaFemina, Pacific Northwest National Laboratory, Battelle Boulevard, P.O. Box 999, MS P7-27, Richland, Washington 99352

47. G. J. Lumetta, Pacific Northwest National Laboratory, Battelle Boulevard, P.O. Box 999, MS P7-25, Richland, Washington 99352

48. J. McGlynn, Science Applications International Corporation, EM-53, 555 Quince Orchard Road, Gaithersburg, Maryland 20878

49. J. O. Moore, Oak Ridge Technical Program Officer, Department of Energy, Oak Ridge Operations, P.O. Box 2001, Oak Ridge, Tennessee 37831-8620

50. J. R. Noble-Dial, Field Office Site Representative, Department of Energy, Oak Ridge Operations, P.O. Box 2001, Oak Ridge, Tennessee, 37831-8620

51. B. M. Rapko, Pacific Northwest National Laboratory, Battelle Boulevard, P.O. Box 999, MS P7-25, Richland, Washington 99352

52. W. W. Schultz, W2S Company, Inc., 5314 Arbustos Court, NE, Albuquerque, New Mexico 87111

53. T. L. Stewart, Pacific Northwest National Laboratory, Battelle Boulevard, P.O. Box 999, MS K9-69, Richland, Washington 99352 
54. D. G. Swanberg, Science Applications International Corporation, MS H0-50, 3250 Port of Benton Boulevard, Richland, Washington 99352

55. J. L. Swanson, 1318 Cottonwood Drive, Richland, Washington 99352

56. I. Tasker, Waste Policy Institute, 555 Quince Orchard Road, Suite 600, Gaithersburg, Maryland 20878-1437

57. L. H. Taylor, Department of Energy, Office of Program Integration, CLOV-EM-43, 19901 Germantown Road, Germantown, Maryland 20874

58. D. J. Temer, Los Alamos National Laboratory, NMT-1 CMR, MS G740, Los Alamos, New Mexico 87545

59. M. C. Thompson, Westinghouse Savannah River Company, Savannah River Technology Center, Building C-140, Room 773-A, Aiken, South Carolina 29808

60. G. F. Vandegrift, Argonne National Laboratory, 9700 South Cass Avenue, Building 205, Argonne, Illinois 60439

61. R. G. Wymer, 188-A Outer Drive, Oak Ridge, Tennessee 37830

62-69. Tanks Focus Area Technical Team, c/o G. C. Notch, Pacific Northwest National Laboratory, Battelle Boulevard, P.0. Box 999, MSIN K9-69, Richland Washington 99352

70. Tanks Focus Area Field Lead, c/o J. A. Frey, U.S. Department of Energy, Richland, Operations Office, P.O. Box 550, MS K8-50, Richland, Washington 99352 